TRANSACTIONS OF THE

AMERICAN MATHEMATICAL SOCIETY

Volume 353, Number 1, Pages 41-76

S 0002-9947(00)02440-5

Article electronically published on April 25, 2000

\title{
PROJECTIVE SETS AND ORDINARY DIFFERENTIAL EQUATIONS
}

\author{
ALESSANDRO ANDRETTA AND ALBERTO MARCONE
}

\begin{abstract}
We prove that for $n \geq 2$ the set of Cauchy problems of dimension $n$ which have a global solution is $\boldsymbol{\Sigma}_{1}^{1}$-complete and that the set of ordinary differential equations which have a global solution for every initial condition is $\Pi_{1}^{1}$-complete. The first result still holds if we restrict ourselves to second order equations (in dimension one). We also prove that for $n \geq 2$ the set of Cauchy problems of dimension $n$ which have a global solution even if we perturb a bit the initial condition is $\Pi_{2}^{1}$-complete.
\end{abstract}

\section{INTRODUCTION AND STATEMENT OF THE MAIN RESUltS}

A Cauchy problem of dimension $n>0$ is a triple $(F, a, \vec{b})$ where $F: \mathbb{R} \times \mathbb{R}^{n} \rightarrow \mathbb{R}^{n}$ is continuous, $a \in \mathbb{R}$ and $\vec{b} \in \mathbb{R}^{n}$. A solution of $(F, a, \vec{b})$ is a differentiable $\varphi: I \rightarrow \mathbb{R}^{n}$ where $I \subseteq \mathbb{R}$ is an open interval, $a \in I, \varphi(a)=\vec{b}$, and $\varphi^{\prime}(x)=F(x, \varphi(x))$ for all $x \in I$. We call $F$ a differential equation of dimension $n$. Let $\mathbf{E q}=C\left(\mathbb{R}^{n+1}, \mathbb{R}^{n}\right)$ be the space of all differential equations of dimension $n$ and $\mathbf{C p}=\mathbf{E q} \times \mathbb{R} \times \mathbb{R}^{n}$ be the space of all Cauchy problems of dimension $n$. (We should really write $\mathbf{E q}{ }^{n}$ and $\mathbf{C p}^{n}$ to stress the dependence on $n$, but this makes the notation rather cumbersome, and we resort to it only when there is danger of confusion.) $C\left(\mathbb{R}^{n+1}, \mathbb{R}^{n}\right)$ is endowed with the compact-open topology, so that Eq and $\mathbf{C p}$ become Polish (i.e. separable and completely metrizable) spaces. Notice that in the definition of $\mathbf{C p}$ we only require $F$ to be continuous: if we put a more stringent constraint on $F$ (e.g. being differentiable, or even just locally Lipschitz in the last $n$ variables), then the solution of $(F, a, \vec{b})$ is unique. Let $\mathcal{U} \subset \mathbf{C p}$ be the set of Cauchy problems which have a unique solution, and let $\mathcal{U}_{\forall} \subset \mathbf{E q}$ be the set of differential equations such that $(F, a, \vec{b}) \in \mathcal{U}$ for every initial condition $(a, \vec{b}) \in \mathbb{R} \times \mathbb{R}^{n}$. In [1] it was proved that $\mathcal{U}$ and $\mathcal{U}_{\forall}$ are both $\boldsymbol{\Pi}_{2}^{0}$-complete and hence Polish spaces. (Actually the proof was given only for the one-dimensional case, but it was noticed in the introduction that the same proof applies to any finite dimension.) Hence establishing whether a Cauchy problem has a unique solution is fairly simple from the point of view of descriptive set theory.

On the other hand it is conventional wisdom that establishing whether a Cauchy problem admits a global solution (a solution $\varphi$ is global if $I=\mathbb{R}$ ) is a difficult task, so it is natural to look for a mathematical explanation of this empirical fact. Let $\mathcal{G} \subset \mathbf{C p}$ be the set of Cauchy problems which have a global solution, and let $\mathcal{G}_{\forall} \subset \mathbf{E q}$ be the set of differential equations such that $(F, a, \vec{b}) \in \mathcal{G}$ for every $(a, \vec{b})$.

Received by the editors March 25, 1998 and, in revised form, September 25, 1998.

1991 Mathematics Subject Classification. Primary 04A15; Secondary 34A12. 
It is not hard (see lemma 1) to show that $\mathcal{G}$ is $\boldsymbol{\Sigma}_{1}^{1}$ and $\mathcal{G}_{\forall}$ is $\boldsymbol{\Pi}_{1}^{1}$. We prove that for $n>1$ these classifications are optimal:

Theorem 1. If $n>1$ then $\mathcal{G}$ is $\boldsymbol{\Sigma}_{1}^{1}$-complete in $\mathbf{C p}$.

Theorem 2. If $n>1$ then $\mathcal{G}_{\forall}$ is $\boldsymbol{\Pi}_{1}^{1}$-complete in $\mathbf{E q}$.

These theorems should be contrasted with the following results from [1], which show that for $n=1$ the "obvious" classifications are much too crude:

Theorem $\mathbf{1}^{\prime}$. If $n=1$ then $\mathcal{G}$ is $\boldsymbol{\Sigma}_{4}^{0}$-complete in $\mathbf{C p}$.

Theorem $\mathbf{2}^{\prime}$. If $n=1$ then $\mathcal{G}_{\forall}$ is $\boldsymbol{\Pi}_{3}^{0}$-complete in $\mathbf{E q}$.

Thus there is no Borel way to tell whether a given Cauchy problem of dimension $n>1$ admits a global solution or whether a given differential equation of dimension $n>1$ has a global solution for every initial condition.

One could also consider the set of Cauchy problems such that every solution is global. By the techniques of [1] this set can be seen to be $\boldsymbol{\Pi}_{2}^{0}$-complete for every $n$.

One can actually sharpen theorem 1 a bit. A Cauchy problem of order $n>0$ is a triple $(\tilde{F}, a, \vec{b})$ where $\tilde{F}: \mathbb{R} \times \mathbb{R}^{n} \rightarrow \mathbb{R}$ is continuous, $a \in \mathbb{R}$ and $\vec{b} \in \mathbb{R}^{n}$. A solution of $(\tilde{F}, a, \vec{b})$ is an $n$-times differentiable $\varphi: I \rightarrow \mathbb{R}$ where $I \subseteq \mathbb{R}$ is an open interval, $a \in I, \varphi^{(i)}(a)=b_{i}$ for $i=0, \ldots, n-1$, and

$$
\varphi^{(n)}(x)=\tilde{F}\left(x, \varphi(x), \varphi^{\prime}(x), \ldots, \varphi^{(n-1)}(x)\right) \quad \text { for all } x \in I .
$$

$C\left(\mathbb{R}^{n+1}, \mathbb{R}\right)$ with the compact-open topology is a Polish space which we denote by $\widetilde{\mathbf{E q}}$ (the space of differential equations of order $n$ ) and $\widetilde{\mathbf{C p}}=\widetilde{\mathbf{E q}} \times \mathbb{R} \times \mathbb{R}^{n}$ (the space of Cauchy problems of order $n$ ) is also Polish. Every differential equation of order $n$ can be identified in a natural way with a differential equation of dimension $n$ : for any $\tilde{F} \in \widetilde{\mathbf{E q}}$ let $F \in \mathbf{E q}$ be defined by $F\left(x, y_{1}, \ldots, y_{n}\right)=\left(y_{2}, \ldots, y_{n}, \tilde{F}\left(x, y_{1}, \ldots, y_{n}\right)\right)$. The map $\widetilde{\mathbf{E q}} \rightarrow \mathbf{E q}, \tilde{F} \mapsto F$, is a homeomorphism between $\widetilde{\mathbf{E q}}$ and a closed subset of Eq and induces a homeomorphism between $\widetilde{\mathbf{C p}}$ and a closed subset of $\mathbf{C p}$. If $\varphi: I \rightarrow \mathbb{R}$ is $C^{n-1}$, the lifting of $\varphi$ is the function $I \rightarrow \mathbb{R}^{n}$,

$$
x \mapsto\left(\varphi(x), \varphi^{\prime}(x), \ldots, \varphi^{(n-1)}(x)\right) .
$$

For $(\tilde{F}, a, \vec{b}) \in \widetilde{\mathbf{C p}}$ the function mapping $\varphi$ to its lifting is a bijection between the set of all solutions of $(\tilde{F}, a, \vec{b})$ and the set of all solutions of $(F, a, \vec{b}) \in \mathbf{C p}$, where $F$ is as above. Therefore the existence of a unique (resp. global) solution of $(\tilde{F}, a, \vec{b})$ is equivalent to the existence of a unique (resp. global) solution of $(F, a, \vec{b})$.

Let $\tilde{\mathcal{U}}, \tilde{\mathcal{G}} \subset \widetilde{\mathbf{C p}}$ and $\tilde{\mathcal{U}}_{\forall}, \tilde{\mathcal{G}_{\forall}} \subset \widetilde{\mathbf{E q}}$ be the analogues of $\mathcal{U}, \mathcal{G}, \mathcal{U}_{\forall}$ and $\mathcal{G}_{\forall}$ for Cauchy problems and differential equations of order $n . \tilde{\mathcal{U}}$ and $\tilde{\mathcal{U}}_{\forall}$ are both $\Pi_{2}^{0}$ (and in fact $\boldsymbol{\Pi}_{2}^{0}$-complete) in $\widetilde{\mathbf{C p}}$ and $\widetilde{\mathbf{E q}}$, respectively. We prove:

Theorem 3. If $n>1$ then $\tilde{\mathcal{G}}$ is $\boldsymbol{\Sigma}_{1}^{1}$-complete in $\widetilde{\mathbf{C p}}$.

In light of our previous discussion theorem 3 is a strengthening of theorem 1 (We do not know whether theorem 2 can be strengthened in a similar way, by proving that $\tilde{\mathcal{G}_{\forall}}$ is $\boldsymbol{\Pi}_{1}^{1}$-complete in $\widetilde{\mathbf{E q}}$ for $n>1$.)

The proofs of theorems 1 2 3 as well as those of 1 and 2, heavily exploit the existence of bifurcation points, so one could ask whether the task of establishing the existence of a global solution becomes easier if one knows in advance that the solution is unique. The answer is indeed positive: in [1] it is shown that for $n=1$, 
$\mathcal{G} \cap \mathcal{U}$ and $\mathcal{G}_{\forall} \cap \mathcal{U}_{\forall}$ are both $\boldsymbol{\Pi}_{2}^{0}$-complete, and here these results are extended to every $n$, by proving:

Theorem 4. For every $n, \mathcal{G} \cap \mathcal{U}$ and $\tilde{\mathcal{G}} \cap \tilde{\mathcal{U}}$ are $\Pi_{2}^{0}$-complete respectively in $\mathcal{U}$ and $\tilde{\mathcal{U}}$; similarly $\mathcal{G}_{\forall} \cap \mathcal{U}_{\forall}$ and $\tilde{\mathcal{G}_{\forall}} \cap \tilde{\mathcal{U}}_{\forall}$ are $\boldsymbol{\Pi}_{2}^{0}$-complete respectively in $\mathcal{U}_{\forall}$ and $\tilde{\mathcal{U}}_{\forall}$.

Other interesting classes of Cauchy problems arise by considering problems that have a given property stably, i.e. even if we perturb a bit the initial condition. The class $\mathcal{S U} \subset \mathbf{C p}$ of stably unique problems is such that $(F, a, \vec{b}) \in \mathcal{S U}$ if and only if

$$
\exists \varepsilon>0 \forall(c, \vec{d})[d((a, \vec{b}),(c, \vec{d}))<\varepsilon \Longrightarrow(F, c, \vec{d}) \in \mathcal{U}],
$$

where $d$ is a compatible metric on $\mathbb{R} \times \mathbb{R}^{n}$. Similarly the class $\mathcal{S G} \subset \mathbf{C p}$ of stably global problems is such that $(F, a, \vec{b}) \in \mathcal{S G}$ if and only if

$$
\exists \varepsilon>0 \forall(c, \vec{d})[d((a, \vec{b}),(c, \vec{d}))<\varepsilon \Longrightarrow(F, c, \vec{d}) \in \mathcal{G}] .
$$

It is not hard (see lemma 1) to show that $\mathcal{S U}$ is $\boldsymbol{\Sigma}_{3}^{0}$ and $\mathcal{S G}$ is $\Pi_{2}^{1}$. We prove:

Theorem 5. For every $n, \mathcal{S U}$ is $\mathbf{\Sigma}_{3}^{0}$-complete in $\mathbf{C p}$.

Theorem 6. If $n=1$ then $\mathcal{S G}$ is $\mathbf{\Sigma}_{4}^{0}$-complete in $\mathbf{C p .}$

Theorem 7. If $n>1$ then $\mathcal{S G}$ is $\mathbf{\Pi}_{2}^{1}$-complete in $\mathbf{C p}$.

Thus, for $n>1, \mathcal{S G}$ provides an example of a "natural" set which appears for the first time at the second level of the projective hierarchy - examples of such sets are not widespread.

Notation and terminology. The notation is quite standard, with the possible exception of $f \uparrow X$ to denote the restriction of the function $f$ to the set $X$, and $\partial(X)$ for the boundary of $X$, for $X$ a subset of some $\mathbb{R}^{n}$.

Some acquaintance with standard arguments of descriptive set theory (see 3 ) is assumed. Here we recall some basic definitions: if $X$ is a Polish space and $A \subset X$, then $A$ is $\boldsymbol{\Sigma}_{1}^{0}$ if it is open, $\boldsymbol{\Pi}_{n}^{0}$ if $X \backslash A$ is $\boldsymbol{\Sigma}_{n}^{0}, \boldsymbol{\Sigma}_{n+1}^{0}$ if it is a countable union of $\boldsymbol{\Pi}_{n}^{0}$ sets (these are the lower levels of the Borel hierarchy). In particular $\boldsymbol{\Sigma}_{2}^{0}=\mathbf{G}_{\delta}$, $\boldsymbol{\Pi}_{3}^{0}=\mathbf{G}_{\delta \sigma}$, etc. $A$ is analytic or $\boldsymbol{\Sigma}_{1}^{1}$ if it is the continuous image of a Borel set, coanalytic or $\boldsymbol{\Pi}_{1}^{1}$ if $X \backslash A$ is $\boldsymbol{\Sigma}_{1}^{1}$, and $\boldsymbol{\Pi}_{2}^{1}$ if it is the complement of a continuous image of a $\boldsymbol{\Sigma}_{1}^{1}$ (these are the lower levels of the projective hierarchy). If $A \subseteq X$ and $B \subseteq Y$ with $X, Y$ Polish, $A \leq_{\mathrm{w}} B$ means that there exists a continuous $f: X \rightarrow Y$ such that $x \in A \Longleftrightarrow f(x) \in B$ (intuitively, $B$ is at least as complex as $A$ ). $A$ is $\boldsymbol{\Sigma}_{1}^{1}$-hard if $B \leq_{\mathrm{W}} A$ for every $B$ which is $\boldsymbol{\Sigma}_{1}^{1}$ in some Polish space $Y$. $A$ is $\boldsymbol{\Sigma}_{1}^{1}$ complete if it is both $\boldsymbol{\Sigma}_{1}^{1}$ and $\boldsymbol{\Sigma}_{1}^{1}$-hard; in this case we can say that $A$ is $\boldsymbol{\Sigma}_{1}^{1}$ and no better. Substituting the other classes defined above in place of $\boldsymbol{\Sigma}_{1}^{1}$, we obtain the definition of e.g. $\boldsymbol{\Pi}_{2}^{1}$-complete. Thus establishing that $A$ is $\boldsymbol{\Sigma}_{1}^{1}$-complete consists of two separate steps: the upper bound on the complexity of $A$ is obtained by showing that $A$ is $\boldsymbol{\Sigma}_{1}^{1}$; the lower bound on this complexity is obtained by showing that $A$ is $\Sigma_{1}^{1}$-hard.

We now turn to differential equations. Unless otherwise noted, a solution $\varphi$ of a differential equation will always be assumed to be non-extendible (i.e. such that there is no extension of $\varphi$ to a larger interval which is still a solution). However, a clarification about our terminology is necessary: according to our definition an element of Eq or $\widetilde{\mathbf{E q}}$ is a function defined on $\mathbb{R}^{n+1}$, but quite often it will be useful 
to consider differential equations $F$ which are defined on a proper subset of $\mathbb{R}^{n+1}$. When this happens, by a solution we mean a function $\varphi$ defined on some interval (not necessarily open) $I$ that satisfies the equation in every point of $I$ and cannot be extended to a larger interval, possibly because $F$ is undefined beyond the endpoint of $\operatorname{graph}(\varphi)$. This does not prevent $\varphi$ from being extended beyond $I$ when the definition of $F$ is extended to a larger domain. If instead $\varphi$ has no chances of being further extended (e.g. because $a^{*}=\sup \operatorname{dom}(\varphi)$ and $\lim _{x \rightarrow a^{*}}\|\varphi(x)\|=+\infty$ ), we say that $\varphi$ dies. We say that $(a, \vec{b})$ is a bifurcation point for $F \in \mathbf{E q}$ if for every $\varepsilon>0$ there exist solutions $\varphi$ and $\psi$ of $(F, a, \vec{b})$ such that $\varphi(x) \neq \psi(x)$ for some $x \in(a-\varepsilon, a+\varepsilon)$; we have a bifurcation point to the right if we can always find $x \in(a, a+\varepsilon)$.

Organization of the paper. The upper bounds on the complexity of the sets are established in section 2: for every $n, \mathcal{G}$ is $\boldsymbol{\Sigma}_{1}^{1}, \mathcal{G}_{\forall}$ is $\boldsymbol{\Pi}_{1}^{1}, \mathcal{G} \cap \mathcal{U}$ and $\mathcal{G}_{\forall} \cap \mathcal{U}_{\forall}$ are $\Pi_{2}^{0}, \mathcal{S U}$ is $\boldsymbol{\Sigma}_{3}^{0}, \mathcal{S G}$ is $\Pi_{2}^{1}$, while for $n=1 \mathcal{S G}$ is $\boldsymbol{\Sigma}_{4}^{0}$. (Notice that these results imply the corresponding results for the subsets of $\widetilde{\mathbf{C p}}$ and $\widetilde{\mathbf{E q}}$.) The proof of theorem 4 is now complete, since in [1] we showed that $\mathcal{G} \cap \mathcal{U}$ and $\mathcal{G}_{\forall} \cap \mathcal{U}_{\forall}$ are $\boldsymbol{\Pi}_{2}^{0}$-hard for $n=1$ and this implies the $\boldsymbol{\Pi}_{2}^{0}$-hardness of these sets for every $n$. For the same reason in the remainder of the paper we will carry out the lower bound parts of the proofs of theorems [2, 3] and [7 in the case $n=2$, since this implies the desired result for every $n>1$. Similarly, to complete the proof of theorem 5 we will show that $\mathcal{S U}$

is $\Sigma_{3}^{0}$-hard for $n=1$. In section 2 we also complete the proofs of theorems 5 and 6 (neither of these theorems involves projective sets, and their proofs are obtained fairly easily from results of [1]). The usual technique for establishing a lower bound for the complexity of a set by proving that it is, say, $\boldsymbol{\Sigma}_{1}^{1}$-hard involves using a set which is already known to be $\boldsymbol{\Sigma}_{1}^{1}$-hard: our proofs will often involve the use of trees, and in section 3 we establish our notation and terminology and prove some lemmas about trees. In section 4 (using some features of the proof of the $\boldsymbol{\Pi}_{3}^{0}$-hardness of $\mathcal{G}_{\forall}$ for $n=1$ contained in [1]) we show that $\mathcal{G}_{\forall}$ is $\boldsymbol{\Pi}_{1}^{1}$-hard for $n=2$ and hence complete the proof of theorem 2. In section [5 we show that $\mathcal{G}$ is $\boldsymbol{\Sigma}_{1}^{1}$-hard for $n=2$ and establish theorem 11. This proof is not necessary from a logical viewpoint, since the result follows from theorem [3 but its main idea is the basic technique for the (much more complex) constructions needed for proving theorems 3 and 7 ? thus we think that it is better to present this idea in the simpler context and later extend it to the more involved situations. In sections 6 and 7 we complete the proofs of theorems [7] and [3, by showing that $\mathcal{S G}$ is $\boldsymbol{\Pi}_{2}^{1}$-hard and that $\tilde{\mathcal{G}}$ is $\boldsymbol{\Sigma}_{1}^{1}$-hard for $n=2$. These proofs require rather technical constructions, whose details can be omitted at first reading and are postponed to section 8

This paper is the natural continuation of [1, and in fact many of the arguments here depend heavily on constructions done there. In the proofs we will often refer to [1, so the reader is advised to have a copy at hand. We refer to [1] and [3] for motivation and background.

\section{UPPER BOUNDS AND TWO EASY LOWER BOUNDS}

Lemma 1. 1. $\mathcal{G}$ is $\boldsymbol{\Sigma}_{1}^{1}$ in $\mathbf{C p}$.

2. $\mathcal{G}_{\forall}$ is $\boldsymbol{\Pi}_{1}^{1}$ in $\mathbf{E q}$.

3. $\mathcal{G} \cap \mathcal{U}$ is $\boldsymbol{\Pi}_{2}^{0}$ in $\mathbf{C p}$ and hence also in $\mathcal{U}$.

4. $\mathcal{G}_{\forall} \cap \mathcal{U}_{\forall}$ is $\boldsymbol{\Pi}_{2}^{0}$ in $\mathbf{E q}$ and hence also in $\mathcal{U}_{\forall}$. 
5. $\mathcal{S U}$ is $\boldsymbol{\Sigma}_{3}^{0}$ in $\mathbf{C p}$.

6. $\mathcal{S G}$ is $\boldsymbol{\Pi}_{2}^{1}$ in $\mathbf{C p}$.

7. If $n=1$ then $\mathcal{S G}$ is $\boldsymbol{\Sigma}_{4}^{0}$ in $\mathbf{C p}$.

Proof. 1. Since

$$
\mathcal{G}=\left\{(F, a, \vec{b}) \in \mathbf{C p} \mid \exists \varphi \in C^{1}\left(\mathbb{R}, \mathbb{R}^{n}\right)(\varphi \text { is a solution of }(F, a, \vec{b}))\right\}
$$

it suffices to show that

$$
S=\left\{(F, a, \vec{b}, \varphi) \in \mathbf{C p} \times C^{1}\left(\mathbb{R}, \mathbb{R}^{n}\right) \mid \varphi \text { is a solution of }(F, a, \vec{b})\right\}
$$

is Borel (here $C^{1}\left(\mathbb{R}, \mathbb{R}^{n}\right)$ is given the Polish topology obtained by identifying every $\varphi$ with $\left.\left(\varphi, \varphi^{\prime}\right) \in C\left(\mathbb{R}, \mathbb{R}^{n}\right)^{2}\right)$. Since $(F, a, \vec{b}, \varphi) \in S$ if and only if $\varphi(a)=\vec{b}$ and $\forall q \in \mathbb{Q} \varphi^{\prime}(q)=F(q, \varphi(q)), S$ is closed.

2. Since

$$
\mathcal{G}_{\forall}=\{F \in \mathbf{E q} \mid \forall(a, \vec{b})(F, a, \vec{b}) \in \mathcal{G}\}
$$

at first sight $\mathcal{G}_{\forall}$ is $\boldsymbol{\Pi}_{2}^{1}$. However, we notice that $F \in \mathcal{G}_{\forall}$ if and only if for every $(a, \vec{b}) \in \mathbb{R} \times \mathbb{R}^{n}$ there exists a solution $\varphi$ of $(F, a, \vec{b})$ whose domain contains the closed interval $[a-1, a+1]$ (since this solution can then be continued by solutions of $(F, a \pm 1, \varphi(a \pm 1))$ and so on). For a fixed $(a, \vec{b})$ the last condition is equivalent to the existence of $M \in \mathbb{N}$ such that $F \in E_{M}$, where $E_{M}$ is the set of $F \in \mathbf{E q}$ for which there exists a solution $\varphi$ of $(F, a, \vec{b})$ such that $\operatorname{dom}(\varphi) \supseteq[a-1, a+1]$ and $\|\varphi \uparrow[a-1, a+1]\|_{\infty} \leq M$. It is now easy to check (e.g. using the Ascoli-Arzelà theorem) that $E_{M}$ is closed and hence $\mathcal{G}_{\forall}$ is $\boldsymbol{\Pi}_{1}^{1}$.

3. If $(F, a, \vec{b}) \in \mathcal{U} \backslash \mathcal{G}$, then its unique solution $\varphi$ has a domain $I$ which is bounded either above or below. For the sake of the argument assume that $I$ is bounded above, and let $c=\sup I$. From the continuity of $F$ and the mean value theorem, it is not hard to see that $\lim _{x \rightarrow c^{-}}\|\varphi(x)\|=+\infty$. For any $q \in \mathbb{Q}$ consider the set of Cauchy problems which have a solution $\varphi$ such that $\lim _{x \rightarrow c^{-}}\|\varphi(x)\|=+\infty$ for some $c \in(a, q]$ : the calculations of the proof of theorem 5.4 of [1] show that this set is closed. Hence $\mathcal{U} \backslash \mathcal{G}$ is $\boldsymbol{\Sigma}_{2}^{0}$ in $\mathcal{U}$.

4. This follows from 3 exactly as in the dimension 1 case (corollary 5.5 of [1]): one uses the fact (lemma 1.3 of [1]) that the projection of a $\Sigma_{2}^{0}$ set (in this case the complement of $\mathcal{G} \cap \mathcal{U}$ ) along a $\mathbf{K}_{\sigma}$ space (in this case $\mathbb{R} \times \mathbb{R}^{n}$ ) is a $\boldsymbol{\Sigma}_{2}^{0}$ set (in this case the complement of $\left.\mathcal{G}_{\forall} \cap \mathcal{U}_{\forall}\right)$.

5. This is immediate from the definition of $\mathcal{S U}$, the fact that $\mathcal{U}$ is $\boldsymbol{\Sigma}_{2}^{0}$, the above mentioned lemma 1.3 of [1], and the fact that we can restrict the quantifier on $\varepsilon$ to $\mathbb{Q}$.

6. This is immediate from the definition of $\mathcal{S G}$ and from the fact that $\mathcal{G}$ is $\boldsymbol{\Sigma}_{1}^{1}$ (again we can search for $\varepsilon$ in $\mathbb{Q}$ ).

7. Let $n=1$. We claim that $(F, a, b) \in \mathcal{S G}$ if and only if

$$
\exists q \in \mathbb{Q}(q>0 \&(F, a, b+q) \in \mathcal{G} \&(F, a, b-q) \in \mathcal{G}) .
$$

Since $\mathcal{G}$ is $\boldsymbol{\Sigma}_{4}^{0}$ by theorem 1 , the claim completes the proof.

For the nontrivial direction of the equivalence let $\varphi_{0}$ and $\varphi_{1}$ be global solutions of $(F, a, b-q)$ and $(F, a, b+q)$ respectively. Pick $\varepsilon>0$ such that $\varepsilon \leq d\left((a, b),\left(x, \varphi_{i}(x)\right)\right)$ for $x \in \mathbb{R}$ and $i=0,1$. If $d((a, b),(c, d))<\varepsilon$, let $\psi$ be a solution of $(F, c, d)$ : in a neighborhood of $c$ we have $\varphi_{0}(x)<\psi(x)<\varphi_{1}(x)$. 
If $\psi(x) \neq \varphi_{i}(x)$ for all $x \in \mathbb{R}$ and $i$, then $\varphi_{0}(x)<\psi(x)<\varphi_{1}(x)$ for every $x$ and $\psi$ is global; otherwise assume $\psi\left(x_{0}\right)=\varphi_{0}\left(x_{0}\right)$ for some $x_{0}>c$ (symmetrical arguments apply to $\varphi_{1}$ and/or $\left.x_{0}<c\right)$ and define

$$
\chi(x)= \begin{cases}\psi(x) & \text { if } x \leq x_{0} \\ \varphi_{0}(x) & \text { if } x>x_{0}\end{cases}
$$

$\chi$ is a global solution of $(F, c, d)$.

Proof of theorem 5. By lemma 1, part 5 and the discussion in the introduction it suffices to prove that $\mathcal{S U}$ is $\boldsymbol{\Sigma}_{3}^{0}$-hard when $n=1$. The proof is similar to that of theorem 4.2 of [1]: we prove that $S_{3}^{\prime} \leq_{\mathrm{w}} \mathcal{S U}$, where

$$
S_{3}^{\prime}=\left\{\alpha \in 2^{\mathbb{N} \times \mathbb{N}} \mid \forall \forall^{\infty} n \exists^{\infty} m \alpha(n, m)=0\right\}
$$

is $\boldsymbol{\Sigma}_{3}^{0}$-complete.

Pick $c_{n}$ 's and $d_{n}$ 's such that $0<d_{n+1}<c_{n}<d_{n}<1$ and $\lim c_{n}=\lim d_{n}=0$. Let $R_{n}=[0,1] \times\left[c_{n}, d_{n}\right]$. Given $\alpha \in 2^{\mathbb{N} \times \mathbb{N}}$ we need to define $F_{\alpha} \in \mathbf{E q}^{1}$. Set $F_{\alpha}=0$ outside $\bigcup_{n} R_{n}$. Define $F_{\alpha}=G_{\alpha_{n}}$ on $R_{n}$ using lemma 2.8 of [1] (see also lemma 5 of the present paper), with $M=2^{-n}$ and $\alpha_{n}$ the sequence $m \mapsto \alpha(n, m)$. The map $\alpha \mapsto F_{\alpha}$ from $2^{\mathbb{N} \times \mathbb{N}}$ to $\mathbf{E} \mathbf{q}^{1}$ is continuous because $\| F_{\alpha}\left\lceil R_{n} \|_{\infty} \rightarrow 0\right.$. This fact insures also the continuity of $F_{\alpha}$ at $(x, 0)$ for any $x \in[0,1]$. Clearly $\alpha \in S_{3}^{\prime}$ if and only if $\left(F_{\alpha}, 0,0\right) \in \mathcal{S U}$.

Usually one searches for the solutions of a given differential equation. In the following lemma, as in many of the constructions throughout the paper, we revert this approach: here we search for a differential equation which has two prescribed functions as solutions. The lemma will be employed in the proof of theorem 6 , but will also be useful in several other occasions throughout the paper.

Lemma 2. Suppose $\chi_{0}, \chi_{1}:\left[a, a_{0}\right] \rightarrow \mathbb{R}$ are $C^{2}$ functions such that $\chi_{0}(a)=\chi_{1}(a)$, $\chi_{0}{ }^{\prime}(a)=\chi_{1}{ }^{\prime}(a)$, and $\chi_{0}(x)<\chi_{1}(x)$ for every $x \in\left(a, a_{0}\right]$. Let $b=\chi_{0}(a)=\chi_{1}(a)$. Then there exists a continuous $F:\left[a, a_{0}\right] \times \mathbb{R} \rightarrow \mathbb{R}$ (which we view as a one dimensional differential equation) such that:

i) $\chi_{0}$ and $\chi_{1}$ are solutions of $(F, a, b)$;

ii) if $\zeta$ is a solution of $(F, a, b)$ distinct from $\chi_{0}$ and $\chi_{1}$, then $\chi_{0}(x)<\zeta(x)<$ $\chi_{1}(x)$ for every $x \in\left(a, a_{0}\right]$;

iii) $F$ is locally Lipschitz in the second variable everywhere but at $(a, b)$, and hence if $(c, d) \neq(a, b)$, then the Cauchy problem $(F, c, d)$ has a unique solution;

iv) for every $(x, y) \in\left[a, a_{0}\right] \times \mathbb{R}$ either $F(x, y) \in\left[\chi_{0}{ }^{\prime}(x), \chi_{1}{ }^{\prime}(x)\right]$ or $F(x, y) \in$ $\left[\chi_{1}{ }^{\prime}(x), \chi_{0}{ }^{\prime}(x)\right]$, depending on which interval is nonempty.

Obviously an analogous statement can be made if $a_{0}<a$ by reverting upper and lower bounds of the intervals considered.

Proof. Define $F$ by

$$
F(x, y)= \begin{cases}\chi_{0}{ }^{\prime}(x) & \text { if } y \leq \chi_{0}(x) ; \\ \frac{y-\chi_{0}(x)}{\chi_{1}(x)-\chi_{0}(x)} \chi_{1}{ }^{\prime}(x)+\frac{\chi_{1}(x)-y}{\chi_{1}(x)-\chi_{0}(x)} \chi_{0}{ }^{\prime}(x) & \text { if } \chi_{0}(x)<y<\chi_{1}(x) ; \\ \chi_{1}{ }^{\prime}(x) & \text { if } y \geq \chi_{1}(x) .\end{cases}
$$

i), iii) and iv) are immediate, so it is enough to check that ii) also holds. 


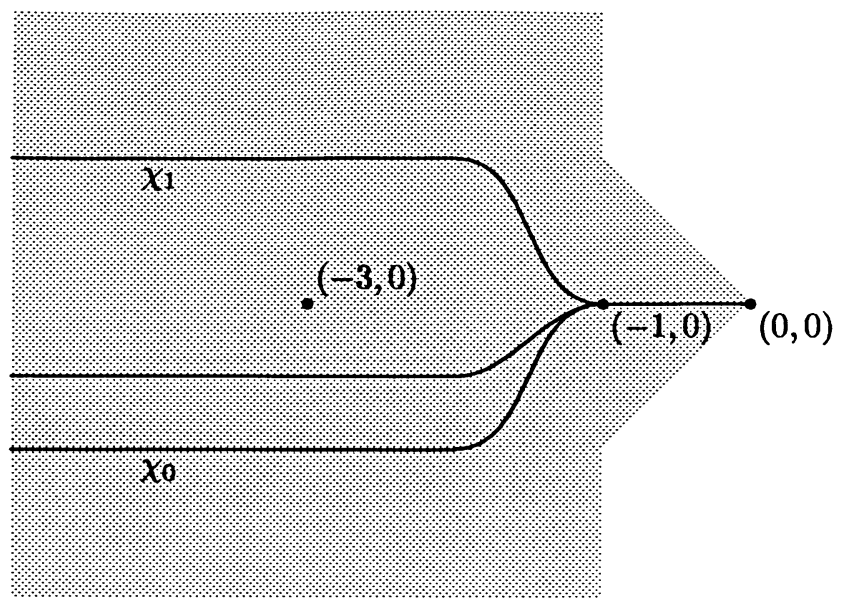

FigURE 1.

Suppose $\zeta$ is a solution of $(F, a, b)$ such that $\chi_{0}(x)<\zeta(x)<\chi_{1}(x)$ does not hold for some $x \in\left(a, a_{0}\right]$. For the sake of the argument let us suppose that $\zeta(x) \geq \chi_{1}(x)$, and let $\varepsilon=\zeta(x)-\chi_{1}(x) \geq 0$. It is immediate to check that $\chi_{1}+\varepsilon$ is a solution of $\tilde{F}$. By iii) (applied to the point $(x, \zeta(x))$ ) we have $\zeta=\chi_{1}+\varepsilon$ and, since $\zeta(a)=b$ we have $\varepsilon=0$ : therefore $\zeta=\chi_{1}$. If $\zeta(x) \leq \chi_{0}(x)$, a similar argument yields $\zeta=\chi_{0}$.

Proof of theorem [6. By lemma [1, part [7, it suffices to prove that $\mathcal{S G}$ is $\boldsymbol{\Sigma}_{4}^{0}$-hard when $n=1$. Let $\mathcal{G}^{+}$be the set of all $(F, a, b)$ which admit a solution $\varphi$ with domain unbounded above, i.e. $[a,+\infty) \subset \operatorname{dom}(\varphi)$. By the proof of lemma 5.7 in 1], which shows that $\mathcal{G}^{+}$is $\boldsymbol{\Sigma}_{4}^{0}$-hard, the set $\mathcal{A}=\left\{F \in \mathbf{E q} \mid F(0,0)=0 \&(F, 0,0) \in \mathcal{G}^{+}\right\}$is also $\boldsymbol{\Sigma}_{4}^{0}$-hard in the Polish space $\{F \in \mathbf{E q} \mid F(0,0)=0\}$, so it is enough to show that $\mathcal{A} \leq_{\mathrm{W}} \mathcal{S G}$.

We define a continuous map Eq $\rightarrow \mathbf{E q}, F \mapsto F^{*}$, such that $F \in \mathcal{A}$ if and only if $\left(F^{*},-3,0\right) \in \mathcal{S G}$. Fix $C^{2}$ functions $\chi_{0}, \chi_{1}:(-\infty,-1] \rightarrow \mathbb{R}$ such that $\chi_{0}(x)=-1$ and $\chi_{1}(x)=1$ for every $x \in(-\infty,-2], \chi_{0}(x)<\chi_{1}(x)$ for every $x \in(-2,-1)$, $\chi_{0}(-1)=\chi_{1}(-1)=0$, and $\chi_{0}{ }^{\prime}(-1)=\chi_{1}{ }^{\prime}(-1)=0$. Lemma 2 implies that there exists $G:(-\infty,-1] \times \mathbb{R} \rightarrow \mathbb{R}$ with $G(0,-1)=0$ such that for any $(a, b)$ with $a \in(-\infty,-1]$ and $\chi_{0}(a) \leq b \leq \chi_{1}(a)$, any solution $\varphi$ of $(G, a, b)$ has domain $(-\infty,-1]$ and is such that $\varphi(-1)=0$. Notice also (from the proof of lemma 2) that $G(-1, y)=0$ for every $y$.

For every $F \in \mathbf{E q}$ such that $F(0,0)=0$ we set $F^{*}=F$ on $[0,+\infty) \times \mathbb{R}$, and $F^{*}=G$ on $(-\infty,-1] \times \mathbb{R}$. The strip $[-1,0] \times \mathbb{R}$ is used as a buffer zone between the two definitions, and we set $F^{*}=0$ on $\{(x, y)|-1 \leq x \leq 0 \&| y \mid \leq x\}$ to make sure that if $\varphi$ is a solution of $\left(F^{*},-1,0\right)$, then $\varphi(0)=0$, so that $\varphi$ is a solution also of $\left(F^{*}, 0,0\right)$. Notice that $F^{*}$ is continuous and has been defined on a closed subset of $\mathbb{R}^{2}$. Using Tietze's extension theorem, we can extend $F^{*}$ to $\mathbb{R}^{2}$ and we can arrange things so that $F \mapsto F^{*}$ is continuous. Figure 1 represents the situation, with the curves being solutions of $F^{*}$ and the shaded area being where the definition of $F^{*}$ does not depend on $F$. Notice that for any $(a, b) \in(-\infty,-2] \times[-1,1]$, if $\varphi$ is a 
solution of $\left(F^{*}, a, b\right)$, then $\operatorname{dom}(\varphi) \supset(-\infty, 0]$ and $\varphi(0)=0$. Therefore $F \in \mathcal{A}$ if and only if $\left(F^{*},-3,0\right) \in \mathcal{S G}$.

It is worth noticing that the above proof could be modified to deduce from theorem 1 (to be proved in section 5) that $\mathcal{S G}$ is $\boldsymbol{\Sigma}_{1}^{1}$-hard when $n>1$ : however, a sharper result will be obtained in section 6 , where $\mathcal{S G}$ will be shown to be $\Pi_{2}^{1}$-hard when $n>1$ (theorem 7).

\section{TREES}

Throughout this paper, except in section [6, by a tree we mean a binary tree, that is, a subset of $2^{<\mathbb{N}}$ (the set of all finite sequences of 0's and 1's) which is closed under initial segments. The length of $s \in 2^{<\mathbb{N}}$ is denoted by $\operatorname{lh}(s)$, if $i<\operatorname{lh}(s)$ the $(i+1)$-th element of $s$ is $s(i)$ and $s \uparrow n$ is the initial segment of $s$ of length $n$, for $n \leq \operatorname{lh}(s)$. Moreover let $s^{-}=s \uparrow(\operatorname{lh}(s)-1) . s \subseteq t$ stands for " $s$ is an initial segment of $t$ ", while $s^{\imath} t$ is " $s$ followed by $t$ ". \langle\rangle is the empty sequence, $\langle 0\rangle$ and $\langle 1\rangle$ are the two sequences of length 1 , while $0^{n}$ and $1^{n}$ denote the two constant sequences of length $n$. The elements of $2^{<\mathbb{N}}$ are ordered lexicographically: $s<_{\operatorname{lex}} t$ means either that $s \subset t$ or else that for some $n<\operatorname{lh}(s), \operatorname{lh}(t)$, we have $s\lceil n=t\lceil n$ and $s(n)<t(n)$.

If $s \in 2^{<\mathbb{N}}$ and $\alpha \in 2^{\mathbb{N}}$ (the set of all infinite sequences of 0's and 1's), $s \subset \alpha$ means that $s$ is an initial segment of $\alpha$ (i.e. that $\alpha \uparrow \operatorname{lh}(s)=s$ ), $s^{\frown} \alpha$ is the element of $2^{\mathbb{N}}$ consisting of $s$ followed by $\alpha . \overline{0}$ and $\overline{1}$ are the constant sequences in $2^{\mathbb{N}}$. We denote by $<_{\text {lex }}$ also the lexicographic order on the elements of $2^{\mathbb{N}}$, so that $\alpha<_{\operatorname{lex}} \beta$ means that for some $n, \alpha\left\lceil n=\beta\left\lceil n\right.\right.$ and $\alpha(n)<\beta(n) . \alpha \in 2^{\mathbb{N}}$ is a branch of the tree $T$ if $\alpha\lceil n \in T$ for every $n \in \mathbb{N}$. [T] is the set of all branches of $T$.

If $T$ is a tree and $n \in \mathbb{N}$, let $T \nmid n=\{s \in T \mid \operatorname{lh}(s) \leq n\}$ and let $T[n]=$ $\{s \in T \mid \operatorname{lh}(s)=n\}$. Any tree can be identified with an element of $2^{2^{<\mathbb{N}}} \cong 2^{\mathbb{N}}$, via its characteristic function. Let $\operatorname{Tr}$ be the set of all trees. The metric

$$
d(S, T)= \begin{cases}2^{-n} & \text { if } T\lceil n=S\lceil n \text { but } T[n+1] \neq S[n+1], \\ 0 & \text { if } S=T\end{cases}
$$

is a complete metric for the topology $\operatorname{Tr}$ has as a subspace of $2^{2^{<N}}$; therefore $\operatorname{Tr}$ is a Polish space. A tree is pruned if every node has at least one extension. Let $\mathrm{PTr} \subseteq \operatorname{Tr}$ be the set of all nonempty pruned trees: $\mathrm{PTr}$ is closed in $\operatorname{Tr}$ and hence a Polish space, with the same complete metric.

Let $\mathrm{S} \subset \mathrm{PTr}$ be the collection of all pruned trees which have at least one branch with infinitely many 1's. It is well known that $\mathrm{S}$ is $\boldsymbol{\Sigma}_{1}^{1}$-complete (see [3, section 27.A], where $\mathrm{S}$ is called $\mathrm{IF}_{2}^{*}$ ) and hence its complement $\mathrm{P} \operatorname{Tr} \backslash \mathrm{S}$ is $\boldsymbol{\Pi}_{1}^{1}$-complete.

In section 6 we will use trees on the product $2 \times 2$, that is, subsets of $(2 \times 2)^{<\mathbb{N}}$ which are closed under initial segments. Actually it is more convenient to think of these trees as subsets $T$ of $2^{<\mathbb{N}} \times 2^{<\mathbb{N}}$ such that if $(s, t) \in T$ then $\ln (s)=\ln (t)$; the closure under initial segments now means that $(s, t) \in T$ and $n \leq \operatorname{lh}(s)$ imply $(s\lceil n, t \uparrow n) \in T$ (we will think of trees on other product spaces in the same way). Let us denote the space of such trees by $\operatorname{Tr}_{2 \times 2}$. It is immediate that $\operatorname{Tr}_{2 \times 2}$

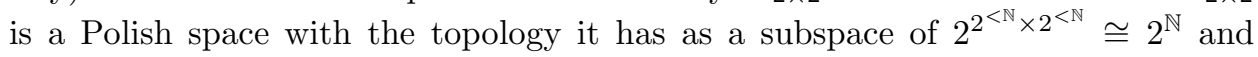
that a complete metric analogous to the one we defined on $\operatorname{Tr}$ can be defined also on $\operatorname{Tr}_{2 \times 2}$. Whenever $T \in \operatorname{Tr}_{2 \times 2}$ and $\alpha \in 2^{\mathbb{N}}$ we can consider the section of $T$ at $\alpha: T(\alpha)=\left\{t \in 2^{<\mathbb{N}} \mid(\alpha \uparrow \operatorname{lh}(t), t) \in T\right\}$, which is a binary tree. Notice that if 
$[T]=\{(\alpha, \beta) \mid \forall n(\alpha\lceil n, \beta\lceil n) \in T\}$, then $[T(\alpha)]$ is the section of $[T]$ determined by $\alpha$, i.e. $\{\beta \mid(\alpha, \beta) \in[T]\}$.

Again we will be interested in pruned trees on $2 \times 2$, which form the Polish space $\mathrm{PTr}_{2 \times 2}$. Notice that $T(\alpha)$ is not necessarily pruned, even if $T \in \mathrm{PTr}_{2 \times 2}$. For this reason we are especially interested in

$$
\begin{aligned}
\mathrm{R} & =\left\{T \in \mathrm{P}_{2 \times 2} \mid \forall \alpha \in 2^{\mathbb{N}} T(\alpha) \in \mathrm{PTr}\right\} \\
& =\left\{T \in \operatorname{PTr}_{2 \times 2} \mid \forall(s, t) \in T \forall i<2 \exists j<2\left(s^{\frown}\langle i\rangle, t^{\frown}\langle j\rangle\right) \in T\right\} .
\end{aligned}
$$

$\mathrm{R}$ is closed in $\mathrm{PTr}_{2 \times 2}$ and thus is a Polish space. Let

$$
\mathrm{P}=\left\{T \in \mathrm{R} \mid \forall \alpha \in 2^{\mathbb{N}} T(\alpha) \in \mathrm{S}\right\} .
$$

Lemma 3. $\mathrm{P}$ is $\boldsymbol{\Pi}_{2}^{1}$-complete in $\mathrm{R}$.

Proof. It is immediate that $\mathrm{P}$ is $\boldsymbol{\Pi}_{2}^{1}$. The proof that it is $\boldsymbol{\Pi}_{2}^{1}$-hard in $\mathrm{R}$ consists of two steps: we will show first that $Q \leq_{W} P$ and then that $Q$ is $\Pi_{2}^{1}$-hard in $\operatorname{Tr}_{2 \times 2}$, where

$$
\mathrm{Q}=\left\{T \in \operatorname{Tr}_{2 \times 2} \mid \forall \alpha \in 2^{\mathbb{N}} T(\alpha) \in \mathrm{S}\right\} .
$$

(Actually, $\mathrm{Q}=\mathrm{P}$ since $\mathrm{Q} \subset \mathrm{R}$, but we use different notations to stress that we view $\mathrm{Q}$ as a subset of $\operatorname{Tr}_{2 \times 2}$ and $\mathrm{P}$ as a subset of $\mathrm{R}$.)

To prove that $\mathrm{Q} \leq_{\mathrm{W}} \mathrm{P}$ consider the continuous map $\operatorname{Tr}_{2 \times 2} \rightarrow \mathrm{R}, T \mapsto T^{*}$, where

$$
\begin{aligned}
T^{*}= & \left\{\left(s^{*}, t^{*}\right) \in 2^{<\mathbb{N}} \times 2^{<\mathbb{N}} \mid \operatorname{lh}\left(s^{*}\right)=\ln \left(t^{*}\right)\right. \\
& \left.\& \exists(s, t) \in T\left[s \subseteq s^{*} \& t^{*}=t^{\frown} 0^{\left(\ln \left(t^{*}\right)-\ln (t)\right)}\right]\right\} .
\end{aligned}
$$

Thus $T^{*}$ is obtained from $T$ by extending every $(s, t) \in T$ with $\left(u, 0^{\operatorname{lh}(u)}\right)$, where $u$ is arbitrary. We will complete the first part of the proof by showing that for every $\alpha \in 2^{\mathbb{N}}, T^{*}(\alpha) \in \mathrm{S}$ if and only if $T(\alpha) \in \mathrm{S}$.

One direction follows immediately from $T \subseteq T^{*}$. For the other direction suppose $\beta \in\left[T^{*}(\alpha)\right]$. If $\beta(m)=1$ then $(\alpha \uparrow(m+1), \beta \uparrow(m+1)) \in T^{*}$ implies $(\alpha \uparrow$ $(m+1), \beta \uparrow(m+1)) \in T$. So, if $\beta$ has infinitely many 1's, $(\alpha\lceil n, \beta\lceil n) \in T$, for every $n$, i.e. $\beta \in[T(\alpha)]$. Thus $T^{*}(\alpha) \in \mathrm{S}$ implies $T(\alpha) \in \mathrm{S}$.

To prove that $\mathrm{Q}$ is $\boldsymbol{\Pi}_{2}^{1}$-hard, let $A \subseteq \mathbb{N}^{\mathbb{N}}$ be $\boldsymbol{\Pi}_{2}^{1}$ : there exists $B \subseteq \mathbb{N}^{\mathbb{N}} \times 2^{\mathbb{N}}$ which is $\boldsymbol{\Sigma}_{1}^{1}$ and such that

$$
\alpha \in A \Longleftrightarrow \forall \beta \in 2^{\mathbb{N}}(\alpha, \beta) \in B .
$$

By [3, proposition 25.2] there exists a tree $T$ on $\mathbb{N} \times 2 \times 2$ such that

$$
\begin{aligned}
(\alpha, \beta) \in B & \Longleftrightarrow \exists \gamma \in 2^{\mathbb{N}}(\gamma \text { has infinitely many } 1 \text { 's \& }(\alpha, \beta, \gamma) \in[T]) \\
& \Longleftrightarrow T(\alpha, \beta) \in \mathrm{S} .
\end{aligned}
$$

Therefore

$$
\alpha \in A \Longleftrightarrow \forall \beta \in 2^{\mathbb{N}} T(\alpha, \beta) \in \mathrm{S} \Longleftrightarrow T(\alpha) \in \mathrm{Q} .
$$

Since the map $\alpha \mapsto T(\alpha)$ is continuous, $A \leq_{\mathrm{W}} \mathrm{Q}$.

In section 6 the following operation on the elements of $\operatorname{Tr}_{2 \times 2}$ will be useful: given $T \in \operatorname{Tr}_{2 \times 2}$, define $\tilde{T}$ so that $(\tilde{s}, \tilde{t}) \in 2^{<\mathbb{N}} \times 2^{<\mathbb{N}}$ belongs to $\tilde{T}$ if and only if either $\tilde{s}=0^{n}$ and $\tilde{t}=1^{n}$ for some $n$, or else there exists $(s, t) \in T$ such that $\tilde{s}=0^{m \frown}\langle 1\rangle^{\frown} s$

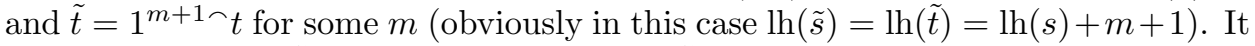
is immediate that $\tilde{T} \in \operatorname{Tr}_{2 \times 2}$ and that $T \mapsto \tilde{T}$ is continuous. The following lemma shows that this operation, restricted to $\mathrm{R}$, preserves being in $\mathrm{P}$ and transforms "not 
being in $\mathrm{P}$ " into a stronger property: if $T \notin \mathrm{P}$ then in every neighborhood of $\overline{0}$ there is an $\alpha$ witnessing $\tilde{T} \notin \mathrm{P}$.

Lemma 4. Let $T \in \mathrm{R}$. Then $\tilde{T} \in \mathrm{R}$, and if $T \in \mathrm{P}$ then $\tilde{T} \in \mathrm{P}$; while if $T \notin \mathrm{P}$ then for every $m$ there exists $\alpha \neq \overline{0}$ such that $\tilde{T}\left(0^{m \frown} \alpha\right) \notin \mathrm{S}$. Therefore

$$
T \in \mathrm{P} \Longleftrightarrow \exists m \forall \alpha \neq \overline{0} \tilde{T}\left(0^{m \frown} \alpha\right) \in \mathrm{S} .
$$

Proof. To show $\tilde{T} \in \mathrm{R}$ let $(\tilde{s}, \tilde{t}) \in \tilde{T}$. If $\tilde{s}=0^{n}$ and $\tilde{t}=1^{n}$, then it is immediate that $\left.\forall i\left(\tilde{s}^{\wedge}\langle i\rangle, \tilde{t}\right\urcorner\langle 1\rangle\right) \in \tilde{T}$. If there exists $(s, t) \in T$ such that $\left.\tilde{s}=0^{m \frown}\langle 1\rangle\right\urcorner s$ and $\tilde{t}=1^{m+1 \frown} t$, then for every $i$ there exists $j$ such that $\left(s^{\wedge}\langle i\rangle, t^{\frown}\langle j\rangle\right) \in T$, whence $\left(\tilde{s}^{\frown}\langle i\rangle, \tilde{t}^{\urcorner}\langle j\rangle\right) \in \tilde{T}$.

Notice that $(\overline{0}, \overline{1}) \in[\tilde{T}]$, and hence $\tilde{T}(\overline{0}) \in \mathrm{S}$, for any $T$. Therefore if $T \in \mathrm{P}$, to show that $\tilde{T} \in \mathrm{P}$ it suffices to show that $\tilde{T}(\tilde{\alpha}) \in \mathrm{S}$ for every $\tilde{\alpha} \neq \overline{0}$. Fix such an $\tilde{\alpha}$,

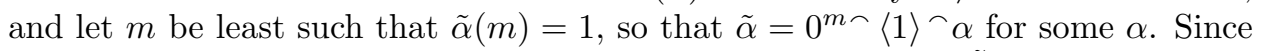
$T(\alpha) \in \mathrm{S}$ there exists $\beta \in[T(\alpha)]$ with infinitely many 1 's. Let $\tilde{\beta}=1^{m+1 \sim} \beta$ : then $\tilde{\beta} \in[\tilde{T}(\tilde{\alpha})]$ and hence $\tilde{T}(\tilde{\alpha}) \in \mathrm{S}$.

If $T \notin \mathrm{P}$ let $\gamma$ be such that $T(\gamma) \notin \mathrm{S}$. Fix $m$, and let $\tilde{\alpha}=0^{m \frown}\langle 1\rangle^{\frown} \gamma$ and $\alpha=\langle 1\rangle^{\frown} \gamma \neq \overline{0}$. It suffices to show that $\tilde{T}\left(0^{m \frown} \alpha\right) \notin \mathrm{S}$, i.e. $\tilde{T}(\tilde{\alpha}) \notin \mathrm{S}$. To prove this let $\tilde{\beta}$ have infinitely many 1's. If $\tilde{\beta}(n)=0$ for some $n \leq m$, then it is clear that $(\tilde{\alpha} \uparrow(n+1), \tilde{\beta} \uparrow(n+1)) \notin \tilde{T}$ and hence $\tilde{\beta} \notin[\tilde{T}(\tilde{\alpha})]$. Hence we may assume that $\tilde{\beta}=1^{m+1} \beta$ for some $\beta$ (which obviously has infinitely many 1's). From $\beta \notin[T(\gamma)]$ it follows that $\tilde{\beta} \notin[\tilde{T}(\tilde{\alpha})]$, and the proof is complete.

\section{4. $\mathcal{G}_{\forall}$ IS $\Pi_{1}^{1}$-HARD FOR $n=2$}

We will define a continuous map $\mathrm{P} \operatorname{Tr} \rightarrow \mathbf{E q}, T \mapsto F_{T}$, witnessing $\mathrm{P} \operatorname{Tr} \backslash \mathrm{S} \leq_{\mathrm{W}} \mathcal{G}_{\forall}$. The function $F_{T}$ will be of the form

$$
F_{T}(x, y, z)=\left(f_{T}(x, y, z), 0\right)
$$

i.e. every solution of $F_{T}$ is contained in a horizontal plane. Therefore we need a continuous map $\mathrm{P} \operatorname{Tr} \rightarrow C\left(\mathbb{R}^{3}, \mathbb{R}\right), T \mapsto f_{T}$, such that

$$
T \notin \mathrm{S} \Longleftrightarrow \forall z f_{T} \uparrow\left(\mathbb{R}^{2} \times\{z\}\right) \in \mathcal{G}_{\forall}^{1} .
$$

The proof relies on the construction used in showing that $\mathcal{G}_{\forall}^{1}$ is $\Pi_{3}^{0}$-hard (see [1] theorem 5.6]). Here is a quick review of that construction (see figure 22).

By a closed rectangle we mean a set $R \subseteq \mathbb{R}^{2}$ of the form $[a, b] \times[c, d]$ with $a<b$ and $c<d$. The segment $[a, b] \times\{c\} \subseteq \mathbb{R}^{2}$ is the basis of $R$. Let us fix $f$ and $\left\{R_{m}\right\}_{m \in \mathbb{N}}$, such that:

a) the $R_{m}$ 's are pairwise disjoint closed rectangles such that every compact subset of the plane intersects only finitely many of them;

b) $f$ is a continuous function defined on the closure of $\mathbb{R}^{2} \backslash \bigcup_{m} R_{m}$ such that $f=0$ on the boundary of each $R_{m}$, the curves in figure 2 are solutions of the first order differential equation $f$, and $f$ has no bifurcation points to the right.

If we extend $f$ to $\mathbb{R}^{2}$ so that $f \geq 0$ on each $R_{m}$, then $f \in \mathcal{G}_{\forall}^{1}$ if and only if $f$ has a bifurcation point to the right on the basis of $R_{m}$, for infinitely many $m$ 's. The proof of theorem 5.6 of [1] is completed by continuously associating to each sequence $\left\{\beta_{m}\right\}$ of elements of $2^{\mathbb{N}}$ an extension of $f$ to $\mathbb{R}^{2}$ so that $f \geq 0$ on each $R_{m}$, 


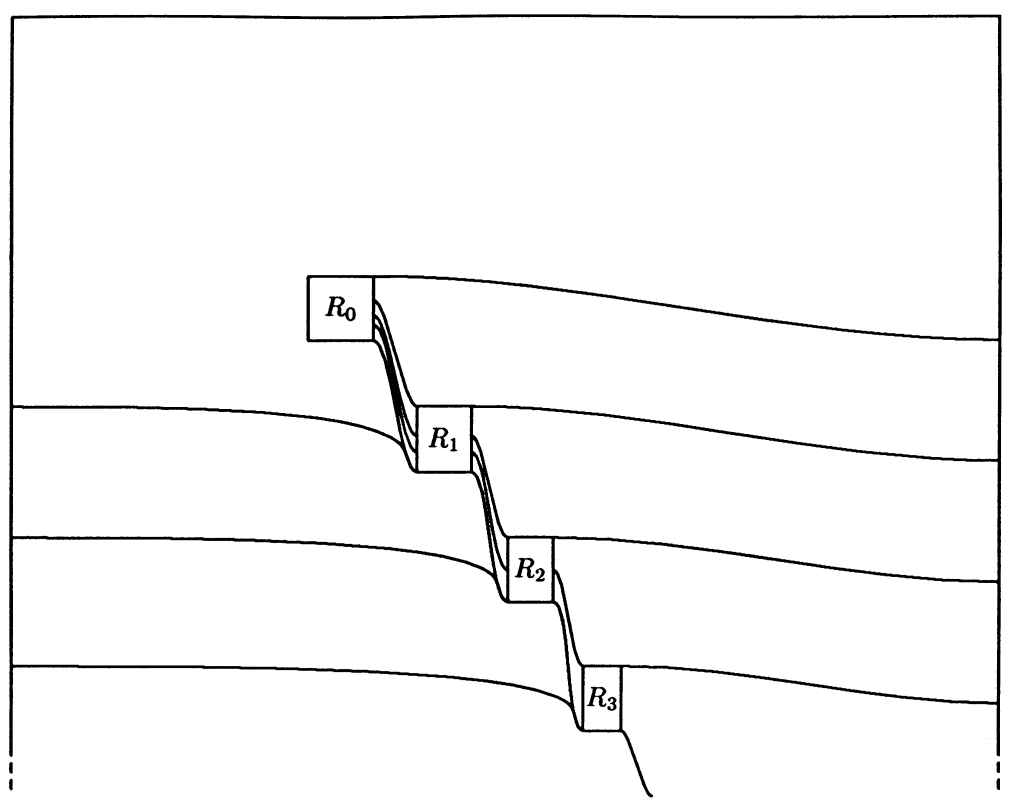

FIGURE 2.

the value on $R_{m}$ depends on $\beta_{m}$ only, and $\beta_{m}$ has infinitely many 1'1 if and only if there are no bifurcation points to the right on the basis of $R_{m}$.

Having one more dimension available, this construction can be carried out on each horizontal plane $z=c$.

Recall the homeomorphism $2^{\mathbb{N}} \rightarrow \mathbf{C}$,

$$
\alpha \mapsto \mathbf{c}_{\alpha}=\sum_{i} \frac{2 \alpha(i)}{3^{i+1}},
$$

where $\mathbf{C} \subset[0,1]$ is Cantor's $1 / 3$-set.

Let $T \in \mathrm{P} \operatorname{Tr}$ and consider the plane $z=\mathbf{c}_{\alpha}$. On this plane we carry out the construction using the sequence $\left\{\beta_{m}\right\}$ defined by

$$
\beta_{m}=\beta_{m}^{\alpha}= \begin{cases}\alpha & \text { if } \alpha \uparrow m \in T ; \\ \overline{0} & \text { if } \alpha \uparrow m \notin T .\end{cases}
$$

Notice that if $\alpha \in[T]$ then $\beta_{m}=\alpha$ for all $m$, while if $\alpha \notin[T], \beta_{m}=\overline{0}$ for $m$ sufficiently large. Therefore if $T \in \mathrm{S}$ and $\beta \in[T]$ has infinitely many 1's, then there exist $a$ and $b$ such that on the plane $z=\mathbf{c}_{\beta},\left(f_{T}, a, b\right) \notin \mathcal{G}^{1} ;$ thus $\left(F_{T}, a, b, \mathbf{c}_{\beta}\right) \notin \mathcal{G}$ and $F_{T} \notin \mathcal{G}_{\forall}$. If instead $T \notin \mathrm{S}$, then $f_{T}$ restricted to any plane $z=c$ with $c \in \mathbf{C}$ belongs to $\mathcal{G}_{\forall}^{1}$. By carefully controlling how $f_{T}$ is defined on the $R_{m}$ 's on the various planes $z=c$ with $c \notin \mathbf{C}$, one can extend it to a continuous function on $\mathbb{R}^{3}$ in such a way that $T \notin \mathrm{S}$ if and only if $F_{T} \in \mathcal{G}_{\forall}$.

Now for the details. For $R=[a, b] \times[c, d]$ a closed rectangle, the upper half of $R$ is the closed rectangle $[a, b] \times[(c+d) / 2, d]$. Define recursively a sequence of closed rectangles by letting $R^{(i)}$ be the upper half of the closed rectangle $\overline{R \backslash \bigcup_{k<i} R^{(k)}}$

\footnotetext{
${ }^{1}$ In [1] we required $\beta_{m}$ to have infinitely many 0's, but this is obviously immaterial.
} 


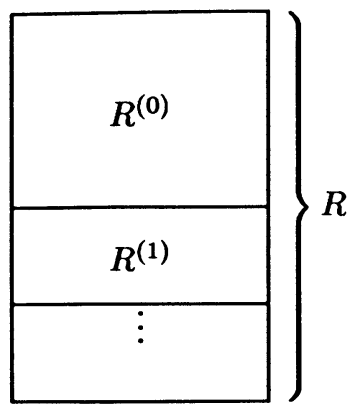

FiguRE 3.

(see figure [3). Thus $R=\overline{\bigcup_{i} R^{(i)}}=\bigcup_{i} R^{(i)} \cup([a, b] \times\{c\})$. A lower portion of $R$ is a closed rectangle of the form $\overline{\bigcup_{i>n} R^{(i)}}=\bigcup_{i \geq n} R^{(i)} \cup([a, b] \times\{c\})$ for some $n$.

We will need a result from [1], lemma 2.8, a version of which is stated below for the reader's convenience (notice that here the roles of 0 and 1 are reversed):

Lemma 5. Let $R=\left[a_{0}, a_{1}\right] \times\left[b_{0}, b_{1}\right]$ be a closed rectangle. There exists a continuous map $2^{\mathbb{N}} \rightarrow C(R, \mathbb{R}), \beta \mapsto G_{\beta}$, such that for every $\beta \in 2^{\mathbb{N}}$ :

i) $G_{\beta}=0$ on $\partial(R)$;

ii) $0 \leq G_{\beta}(x, y) \leq 1$ for every $(x, y) \in R$;

iii) for every $(a, b) \in R$ with $b>b_{0}$ the Cauchy problem $\left(G_{\beta}, a, b\right)$ has a unique solution;

iv) for every $a \in\left[a_{0}, a_{1}\right]$, the Cauchy problem $\left(G_{\beta}, a, b_{0}\right)$ has a unique solution if and only if $\beta$ has infinitely many 1's;

v) $G_{\beta} \uparrow R^{(i)}$ depends only on $\beta(i)$;

vi) if $G$ coincides with $G_{\beta}$ on a lower portion of $R$, then $\left(G, a, b_{0}\right) \in \mathcal{U}$ if and only if $\left(G_{\beta}, a, b_{0}\right) \in \mathcal{U}$ for every $a \in\left[a_{0}, a_{1}\right]$.

Applying lemma 5 in the construction of figure 2, we obtain:

c) the function $\beta_{m} \mapsto f \uparrow R_{m}$ is a continuous map from $2^{\mathbb{N}}$ to $C\left(R_{m}, \mathbb{R}\right)$;

d) $f \uparrow R_{m}^{(i)}$ depends on $\beta_{m}(i)$ only;

e) if $g: \mathbb{R}^{2} \rightarrow \mathbb{R}$ is continuous, coincides with $f$ outside $\bigcup_{m} R_{m}$ and on a lower portion of each $R_{m}$, and $g\left\lceil R_{m} \geq 0\right.$ for each $m$, then $g \in \mathcal{G}_{\forall}^{1}$ if and only if $f \in \mathcal{G}_{\forall}^{1}$.

Going back to the present construction in $\mathbb{R}^{3}$, for every $s \in 2^{<\mathbb{N}}$ let

$$
\mathbf{c}_{s}^{0}=\sum_{i<\operatorname{lh}(s)} \frac{2 s(i)}{3^{i+1}} \quad \text { and } \quad \mathbf{c}_{s}^{1}=\mathbf{c}_{s}^{0}+\frac{1}{3^{\operatorname{lh}(s)}},
$$

so that

- $\mathbf{c}_{s}^{0}=\mathbf{c}_{s^{\circ} \overline{0}}$ and $\mathbf{c}_{s}^{1}=\mathbf{c}_{s^{\wedge} \overline{1}}$;

- $\mathbf{c}_{\alpha} \in\left[\mathbf{c}_{s}^{0}, \mathbf{c}_{s}^{1}\right]$ if and only if $s \subset \alpha$;

- if $z \in[0,1]$ then $z \notin \mathbf{C}$ if and only if there exists $s \in 2^{<\mathbb{N}}$ such that $z \in$ $\left(\mathbf{c}_{s^{\frown}\langle 0\rangle}^{1}, \mathbf{c}_{s^{\frown}\langle 1\rangle}^{0}\right)$.

Notice (using the features of the construction and of the Cantor set we mentioned above) that $f_{T}$ is continuous on $\mathbb{R}^{2} \times \mathbf{C}$, which is a closed subset of $\mathbb{R}^{3}$. Notice also that the map $\mathrm{PTr} \rightarrow C\left(\mathbb{R}^{2} \times \mathbf{C}, \mathbb{R}\right), T \mapsto f_{T} \uparrow\left(\mathbb{R}^{2} \times \mathbf{C}\right)$, is continuous: if 
$T\left\lceil n=S\left\lceil n\right.\right.$ then $F_{T}$ and $F_{S}$ coincide on any set of the form $A \times \mathbf{C}$ such that $A$ is disjoint from $\bigcup_{m \geq n} R_{m}$. Moreover for every $\alpha \in 2^{<\mathbb{N}}$, we have that

$$
f_{T} \uparrow\left(\mathbb{R}^{2} \times\left\{\mathbf{c}_{\alpha}\right\}\right) \in \mathcal{G}_{\forall}^{1} \Longleftrightarrow \alpha \notin[T] \vee \alpha \text { has finitely many 1's. }
$$

Now we need to define $f_{T}(x, y, z)$ when $z \notin \mathbf{C}$. If $(x, y) \notin \bigcup_{m} R_{m}$ we define $f_{T}(x, y, z)$ as in the construction of figure 2 (we are using the fact that outside $\bigcup_{m} R_{m}$ the definition does not depend on $\left.\left\{\beta_{m}\right\}\right)$. If $(x, y) \in \bigcup_{m} R_{m}$ and $z<0$, let $f_{T}(x, y, z)=f_{T}(x, y, 0)$ (which has been defined because $0=\mathbf{c}_{\overline{0}} \in \mathbf{C}$ ), so that $f_{T} \uparrow\left(\mathbb{R}^{2} \times\{z\}\right) \in \mathcal{G}_{\forall}^{1}$. Similarly, if $z>1$ let $f_{T}(x, y, z)=f_{T}(x, y, 1)$, so that $f_{T} \uparrow\left(\mathbb{R}^{2} \times\{z\}\right) \in \mathcal{G}_{\forall}^{1}$ if and only if $\overline{1} \notin[T]$.

We are left with defining $f_{T}$ on $\bigcup_{s, m} R_{m} \times\left(\mathbf{c}_{s^{\prime}<\langle 0\rangle}^{1}, \mathbf{c}_{s^{\circ}\langle 1\rangle}^{0}\right)$. Fix $s \in 2^{<\mathbb{N}}$ and $m \geq 0$ : let $\left\{a_{i}\right\}$ be a strictly decreasing sequence converging to $\mathbf{c}_{s \frown\langle 0\rangle}^{1}$ such that $a_{0}<\mathbf{c}_{s^{\wedge}\langle 1\rangle}^{0}$. Let $i \geq 0$ : for $z \in\left[a_{i}, \mathbf{c}_{s^{\frown}\langle\langle 1\rangle}^{0}\right)$ and $(x, y) \in R_{m}^{(i)}$, let $f_{T}(x, y, z)=$ $f_{T}\left(x, y, \mathbf{c}_{s^{\prec}\langle 1\rangle}^{0}\right)$. Thus $f_{T}$ is defined on the closed set $\left(R_{m} \times\left\{\mathbf{c}_{s^{\prec}\langle 0\rangle}^{1}, \mathbf{c}_{s^{\wedge}\langle 1\rangle}^{0}\right\}\right) \cup$ $\bigcup_{i}\left(R_{m}^{(i)} \times\left[a_{i}, \mathbf{c}_{s^{-}\langle 1\rangle}^{0}\right)\right)$. By Tietze's theorem extend $f_{T}$ to $R_{m} \times\left[\mathbf{c}_{s^{\wedge}\langle 0\rangle}^{1}, \mathbf{c}_{s^{\circ}\langle 1\rangle}^{0}\right]$ with the proviso that $f_{T}$ should be nonnegative everywhere and 0 on $\partial\left(R_{m}\right) \times$ $\left[\mathbf{c}_{s^{\prime}\langle 0\rangle}^{1}, \mathbf{c}_{s^{\prec}\langle 1\rangle}^{0}\right]$. This construction, which completes the definition of $f_{T}$ on $R_{m} \times$ $\left(\mathbf{c}_{s^{\prec}\langle 0\rangle}^{1}, \mathbf{c}_{s^{\frown}\langle 1\rangle}^{0}\right)$, depends only on the values of $f_{T}$ on $R_{m} \times\left\{\mathbf{c}_{s^{\prec}\langle 0\rangle}^{1}, \mathbf{c}_{s^{\frown}\langle\langle 1\rangle}^{0}\right\}$, which in turn depend only on the $\beta_{m}$ 's used in the planes $z=\mathbf{c}_{s^{-}\langle 0\rangle}^{1}$ and $z=\mathbf{c}_{s^{-}\langle 1\rangle}^{0}$, i.e. on whether $\left.s^{\frown}\langle 0\rangle\right\urcorner 1\left\lceil m\right.$ and $\left.s^{\frown}\langle 1\rangle\right\urcorner 0\left\lceil m\right.$ belong to $T$. Let $c \in\left(\mathbf{c}_{s^{\prec}\langle 0\rangle}^{1}, \mathbf{c}_{s^{\frown}\langle 1\rangle}^{0}\right)$ and let $\pi$ be the horizontal plane $z=c$. Then

$$
\pi \cap \bigcup_{i}\left(R_{m}^{(i)} \times\left[a_{i}, \mathbf{c}_{s \smile\langle 1\rangle}^{0}\right)\right)=\bigcup_{i \geq i_{0}} R_{m}^{(i)} \times\{c\}
$$

where $i_{0}$ is least such that $a_{i_{0}} \leq c$. Hence $f_{T}(x, y, c)=f_{T}\left(x, y, \mathbf{c}_{s^{\wedge}\langle 1\rangle}^{0}\right)$ for all $(x, y) \in \bigcup_{i \geq i_{0}} R_{m}^{(i)}$. As this is true of every $R_{m}$, by 国) above $f_{T} \uparrow\left(\mathbb{R}^{2} \times\{c\}\right) \in \mathcal{G}_{\forall}^{1}$ if and only if $f_{T}\left\lceil\left(\mathbb{R}^{2} \times\left\{\mathbf{c}_{s^{\prec}\langle 1\rangle}^{0}\right\}\right) \in \mathcal{G}_{\forall}^{1}\right.$. (Incidentally, since $\mathbf{c}_{s^{\prec}\langle 1\rangle}^{0}=\mathbf{c}_{\left.s^{\prec}\langle 1\rangle\right\urcorner \overline{0}}$, the latter always holds.) By repeating this argument for every $s \in 2^{<\mathbb{N}}$ we obtain a continuous function $f_{T}: \mathbb{R}^{3} \rightarrow \mathbb{R}$.

By construction if $f_{T} \uparrow\left(\mathbb{R}^{2} \times\{c\}\right) \notin \mathcal{G}_{\forall}^{1}$, then there exists $\alpha \in[T]$ such that $f_{T} \uparrow\left(\mathbb{R}^{2} \times\left\{\mathbf{c}_{\alpha}\right\}\right) \notin \mathcal{G}_{\forall}^{1}$, which implies that $\alpha$ has infinitely many 1 's and hence $T \in \mathrm{S}$. Vice versa, if $T \in \mathrm{S}$ and $\alpha \in[T]$ witnesses this, then $f_{T} \uparrow\left(\mathbb{R}^{2} \times\left\{\mathbf{c}_{\alpha}\right\}\right) \notin \mathcal{G}_{\forall}^{1}$. Therefore

$$
T \notin \mathrm{S} \Longleftrightarrow \forall z f_{T} \uparrow\left(\mathbb{R}^{2} \times\{z\}\right) \in \mathcal{G}_{\forall}^{1}
$$

as required.

Finally we check that the map $T \mapsto f_{T}$ is continuous. Let $T, S \in \mathrm{P} T r$. By construction $f_{T}$ and $f_{S}$ agree outside $\bigcup_{m} R_{m} \times \mathbb{R}$. If moreover $T \uparrow n=S \uparrow n$, then $f_{T}$ and $f_{S}$ agree also on $\bigcup_{m<n} R_{m} \times \mathbb{R}$. Therefore given a compact $K \subset \mathbb{R}^{2}$, by 国) above $K$ intersects only finitely many $R_{m}$ 's and, if $n$ is sufficiently large,

$$
T \uparrow n=S \uparrow n \Longrightarrow f_{T} \uparrow(K \times \mathbb{R})=f_{S} \uparrow(K \times \mathbb{R}) .
$$

\section{5. $\mathcal{G}$ IS $\boldsymbol{\Sigma}_{1}^{1}$-HARD FOR $n=2$}

We will show $\mathrm{S} \leq_{\mathrm{W}} \mathcal{G}$ by defining a continuous map $\mathrm{PTr} \rightarrow \mathbf{E q}^{2}, T \mapsto F_{T}$, such that $T \in \mathrm{S} \Longleftrightarrow\left(F_{T}, 0,0,0\right) \in \mathcal{G}$. 


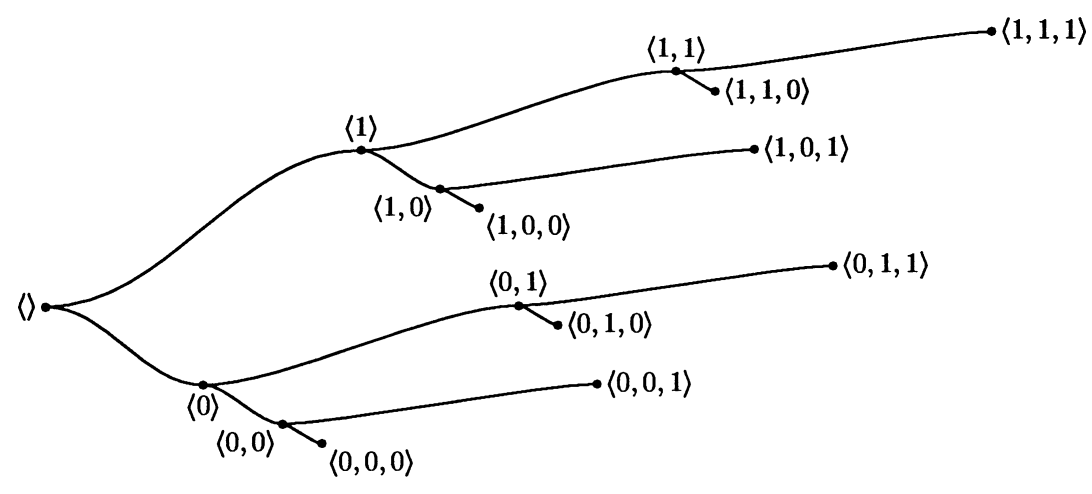

FigURE 4.

First we identify every $T \in \mathrm{PTr}$ with a closed subset of $\mathbb{R}^{3}$. For $s \in 2^{<\mathbb{N}}$ let $\hat{s} \in \mathbb{R}^{<\mathbb{N}}$ be such that $\ln (\hat{s})=\operatorname{lh}(s)$ and

$$
\hat{s}(i)= \begin{cases}2^{-i-1} & \text { if } s(i)=0 \\ 1 & \text { if } s(i)=1\end{cases}
$$

If $\alpha \in 2^{\mathbb{N}}, \hat{\alpha} \in \mathbb{R}^{\mathbb{N}}$ is defined analogously (so that if $s=\alpha \uparrow(i+1), \hat{\alpha}(i)=\hat{s}(i)$ ). Let $x_{s}=\sum_{i<\operatorname{lh}(s)} \hat{s}(i) \in[0,+\infty)$ and $x_{\alpha}=\sum_{i} \hat{\alpha}(i) \in[1,+\infty]$. The basic properties of this construction are:

a) $s \subset t$ implies $x_{s}<x_{t}$;

b) $s \neq t$ implies $x_{s} \neq x_{t}$;

c) $x_{\alpha}=+\infty$ if and only if $\alpha$ has infinitely many 1's.

For $s \in 2^{<\mathbb{N}}$ let $P_{s}=\left(x_{s}, y_{s}, \operatorname{lh}(s)\right)$ where:

- $y_{\langle\rangle}=0$

- if $\operatorname{lh}(s)=\operatorname{lh}(t)$ and $s<_{\operatorname{lex}} t$, then $y_{s}<y_{t}$;

- $y_{s} \sim\langle 0\rangle<y_{s}<y_{s} \sim\langle 1\rangle$.

Therefore $s<_{\text {lex }} t$ and $s \not \subset t$ imply $y_{s}<y_{t}$.

Now that we have identified the vertices of $T$ with the points $P_{s}$, we must define edges between $P_{s}$ and $P_{s} \prec\langle 1\rangle$. Since we will be dealing with functions with values in $\mathbb{R}^{2}$, the following convention will be handy: if $\psi$ is such a function, $\psi^{\mathbf{1}}$ and $\psi^{\mathbf{2}}$ are its two components, i.e. $\psi(x)=\left(\psi^{\mathbf{1}}(x), \psi^{\mathbf{2}}(x)\right)$. For $s \in 2^{<\mathbb{N}}$ with $\operatorname{lh}(s)>0$ recall that $s^{-}=s \uparrow(\operatorname{lh}(s)-1)$ and define a family of $C^{2}$ functions $\varphi_{s}:\left[x_{s^{-}}, x_{s}\right] \rightarrow \mathbb{R}^{2}$ such that:

i) $\varphi_{s}\left(x_{s^{-}}\right)=\left(y_{s^{-}}, \operatorname{lh}(s)-1\right)$ and $\varphi_{s}\left(x_{s}\right)=\left(y_{s}, \operatorname{lh}(s)\right)$ (so that the graph of $\varphi_{s}$ connects $P_{s^{-}}$with $\left.P_{s}\right)$;

ii) $\varphi_{s}^{\mathbf{2}}$ is strictly increasing; if $s(\operatorname{lh}(s)-1)=0$, then $\varphi_{s}^{\mathbf{1}}$ is strictly decreasing; if $s(\operatorname{lh}(s)-1)=1$, then $\varphi_{s}^{\mathbf{1}}$ is strictly increasing;

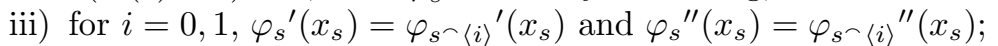

iv) for $i=0,1, \varphi_{\langle i\rangle}{ }^{\prime}(0)=\varphi_{\langle i\rangle}{ }^{\prime \prime}(0)=(0,0)$.

Figure 4 represents the graphs of the $\varphi_{s}^{\mathbf{1}}$ 's for $\ln (s) \leq 3$ : the points $\left(x_{s}, y_{s}\right)$ are labeled by $s$. The actual definitions of the $y_{s}$ 's and $\varphi_{s}$ 's are irrelevant here - the only important thing is to observe that $y_{s}$ 's and $\varphi_{s}$ 's as above do exist. Notice that from the above conditions it follows that if $s<_{\text {lex }} t, s \not \subset t$ and $x \in \operatorname{dom}\left(\varphi_{s}\right) \cap \operatorname{dom}\left(\varphi_{t}\right)$, 
then $\varphi_{s}^{\mathbf{1}}(x)<\varphi_{t}^{\mathbf{1}}(x)$ (except when $s^{-}=t^{-}$and $x=x_{s^{-}}$). Define also $\varphi_{\langle\rangle}$: $(-\infty, 0] \rightarrow \mathbb{R}^{2}$ by setting $\varphi_{\langle\rangle}(x)=(0,0)$ for every $x \leq 0$.

For every $\alpha \in 2^{\mathbb{N}}$ we now define $\varphi_{\alpha}:\left(-\infty, x_{\alpha}\right) \rightarrow \mathbb{R}^{2}$ by setting

$$
\varphi_{\alpha}(x)= \begin{cases}\varphi_{\langle\rangle}(x) & \text { if } x<0 ; \\ \varphi_{\alpha \uparrow(n+1)}(x) & \text { if } x \in\left[x_{\alpha \uparrow n}, x_{\alpha \uparrow(n+1)}\right) .\end{cases}
$$

The last two conditions on the $\varphi_{s}$ 's imply that $\varphi_{\alpha}$ is $C^{2}$. Moreover we have

if $n$ is such that $\alpha \uparrow n=\beta\left\lceil n\right.$ but $\alpha(n)<\beta(n)$, then $\varphi_{\alpha}(x)=$ $\varphi_{\beta}(x)$ for every $x \in\left(-\infty, x_{\alpha \nmid n}\right]$, and $\varphi_{\alpha}^{1}(x)<\varphi_{\beta}^{1}(x)$ (in particular $\left.\varphi_{\alpha}(x) \neq \varphi_{\beta}(x)\right)$ for every $x \in\left(x_{\alpha \mid n}, \min \left(x_{\alpha}, x_{\beta}\right)\right)$.

Therefore whenever $\varphi_{\alpha}(x)=\varphi_{\beta}(x)$ holds, we have also $\varphi_{\alpha}{ }^{\prime}(x)=\varphi_{\beta}{ }^{\prime}(x)$.

Notice that if $\alpha \in[T]$ has finitely many 1's, then $x_{\alpha}<+\infty$ and $\lim _{x \rightarrow x_{\alpha}}-\varphi_{\alpha}^{2}(x)$ $=+\infty$; hence if $\varphi$ is continuous such that $\left(-\infty, x_{\alpha}\right) \subseteq \operatorname{dom}(\varphi)$ and $\varphi\left(x_{\alpha \nmid n}\right)=$ $\varphi_{\alpha}\left(x_{\alpha \mid n}\right)$ for every $n$, then $\operatorname{dom}(\varphi)=\left(-\infty, x_{\alpha}\right)$; i.e. $\varphi$ cannot be further extended to the right.

Our goal is to define $F_{T}$ so that for every $\alpha \in[T], \varphi_{\alpha}$ is a solution of $\left(F_{T}, 0,0,0\right)$. Let

$$
C=C_{T}=\bigcup_{\alpha \in[T]} \operatorname{graph}\left(\varphi_{\alpha}\right)=\left\{(x, y, z) \mid \exists \alpha \in[T]\left(x<x_{\alpha} \& \varphi_{\alpha}(x)=(y, z)\right)\right\} .
$$

If we are to achieve our goal, then the definition of $F_{T} \uparrow C$ must be:

$\left.{ }^{*}\right) \quad F_{T}(x, y, z)=\varphi_{\alpha}{ }^{\prime}(x)$ for some (every) $\alpha \in[T]$ such that $\varphi_{\alpha}(x)=(y, z)$.

If $T \in \mathrm{S}$ there exists $\alpha \in[T]$ with infinitely many 1's and therefore with $\operatorname{dom}\left(\varphi_{\alpha}\right)=$ $\mathbb{R}$; thus $\left(F_{T}, 0,0,0\right) \in \mathcal{G}$. The only problem left is to define $F_{T}$ on $\mathbb{R}^{3} \backslash C$. As $C$ is closed in $\mathbb{R}^{3}$, one is tempted to use Tietze's theorem to extend $F_{T} \uparrow C$ to $\mathbb{R}^{3}$. But this naive approach does not work, as we might introduce new global solutions of $\left(F_{T}, 0,0,0\right)$ even when $T \notin \mathrm{S}$ (so that $\left(F_{T}, 0,0,0\right) \in \mathcal{G}$ would not imply $T \in \mathrm{S}$ ). In extending $F_{T}$ from $C$ to $\mathbb{R}^{3}$ a new solution $\varphi$ can be introduced in two ways. Firstly, if $s^{\frown}\langle 0\rangle, s^{\frown}\langle 1\rangle \in T$, then $P_{s}$ is a bifurcation point to the right for $F_{T}$; hence there are new $\varphi$ 's going through $P_{s}$-in fact there is a continuum of them. Secondly, on $\operatorname{graph}\left(\varphi_{s}\right) \backslash\left\{P_{s^{-}}\right\}$there can be other bifurcation points to the right for $F_{T}$ and hence new $\varphi$ 's. The $\varphi$ 's of the second kind can be easily disposed of by requiring $F_{T}$ to be locally Lipschitz on a tubular neighborhood of $\operatorname{graph}\left(\varphi_{s}\right) \backslash\left\{P_{s^{-}}\right\}$. Although the $\varphi$ 's coming from the bifurcation points $P_{s}$ cannot be eliminated, they can be tamed by making sure they will either die because of a vertical asymptote, or else they will merge into $\varphi_{s} \prec\langle 0\rangle$ or $\varphi_{s} \prec\langle 1\rangle$. To see intuitively (for the detailed construction see claim [6] below) how this can be achieved, fix $a_{0} \in\left(x_{s}, x_{s}<\langle 0\rangle\right)$ and imagine a film-soap surface whose boundary are (the graphs) of $\varphi_{s}\langle 0\rangle$ and $\varphi_{s \sim\langle 1\rangle}$ both restricted to $\left[x_{s}, a_{0}\right]$, and the segment $I$ from $\varphi_{s<\langle 0\rangle}\left(a_{0}\right)$ to $\varphi_{s \sim\langle 1\rangle}\left(a_{0}\right)$. Now imagine continuously deforming $I$ by pulling its midpoint upwards to $+\infty$ and leftwards to some $\left(a^{*}, b^{*}\right) \in\left(x_{s}, a_{0}\right) \times\left(\varphi_{s^{\wedge}\langle 0\rangle}^{1}\left(a^{*}\right), \varphi_{s^{\wedge}\langle 1\rangle}^{1}\left(a^{*}\right)\right)$. The film-soap surface gets stretched to a limit surface $S$ which loosely looks like the graph of a function of two variables with a pole. One can define $F_{T}$ on $S$ and make sure that every solution $\varphi$ of $F_{T}$ passing through $P_{s}$ lives on $S$. One of these $\varphi$ 's will have an asymptote as it approaches $a^{*}$, and the others will be forced to choose a side and merge either into $\varphi_{s^{\prec}\langle 0\rangle}$ or into $\varphi_{s}\langle 1\rangle$. In order to make sure that even after we extend $F_{T}$ to $\mathbb{R}^{3}, S$ contains every solution $\varphi$ passing through $P_{s}, S$ has 
to be thickened to some set $N$ obtained by translating $S$ in the direction of both the $y$ and the $z$ axis by $\pm \varepsilon$. By making sure $F_{T}$ is locally Lipschitz in the interior of $N$, we will guarantee that no $\varphi$ jumps out of $S$. There is one more problem though: suppose $F_{T}$ was defined on some tubular neighborhood $M$ of $\varphi_{s}$, and on some thickening $N$ of the surface $S$ given by $\varphi_{s}\langle 0\rangle$ and $\varphi_{s \sim\langle 1\rangle}$; then we must make sure that the definitions of $F_{T}$ agree on $M \cap N$. To overcome this problem we sharpen $M$ around $P_{s}$ so as to make it look like a pencil with tip $P_{s}$, so that now $M \cap N=\left\{P_{s}\right\}$. We call such an $M$ a pinched neighborhood of $\varphi_{s}$ (see below for the precise definition), because one can think it has been obtained from a tubular neighborhood by squeezing it hard around $P_{s}$. Finally $F_{T}$ is extended to what's left of $\mathbb{R}^{3}$ using Tietze's theorem.

Here come the details. $F_{T}$ will satisfy:

a) if $\alpha \in[T]$ then $\varphi_{\alpha}$ is a solution of $\left(F_{T}, 0,0,0\right)$;

b) if $\varphi$ is a global solution of $\left(F_{T}, 0,0,0\right)$, then there exists a unique $\alpha \in[T]$ with infinitely many 1 's such that $\varphi\left(x_{\alpha \nmid n}\right)=\varphi_{\alpha}\left(x_{\alpha \nmid n}\right)$ for every $n$.

Therefore $\left(F_{T}, 0,0,0\right) \in \mathcal{G}$ if and only if $T \in \mathrm{S}$. Thus the proof will be complete, provided that the map $T \mapsto F_{T}$ is continuous.

We will now make more precise the notion of pinched neighborhood of a curve or of a surface in $\mathbb{R}^{3}$. This notion will be useful in other parts of this paper. Rather than giving an abstract definition of pinched neighborhood we give a couple of examples. Our first example is given by a pinched neighborhood of a surface $S$ which is the graph of a continuous function $G: A \rightarrow \mathbb{R}$ where $A \subseteq(-\infty, a] \times \mathbb{R}$ for some $a \in \mathbb{R}$ is open or closed. A pinched neighborhood of $S$ along the vertical plane $\pi$ of equation $x=a$ is a set of the form

$$
N=\{(x, y, z)|(x, y) \in A \&| z-G(x, y) \mid \leq \eta \cdot(a-x)\},
$$

where $\eta>0$. Notice that if there exists $b$ such that $(a, b) \in A, N$ is not an actual neighborhood of $S$ (because $(a, b, G(a, b))$ does not belong to the interior of $N$ ), but we can think that it is the result of taking a standard closed neighborhood of $S$ of fixed thickness and then "pinching" it along $\pi$, so that its thickness decreases as we approach $\pi$. Then

$$
N \cap \pi=S \cap \pi=\{(x, y, z) \mid(x, y) \in A \& x=a \& z=G(x, y)\} .
$$

It is easy to check the following:

Let $F: N \rightarrow \mathbb{R}^{2}$ be continuous, and suppose every solution $\varphi$ of $(F, x, y$, $G(x, y))$ lies on $S$, for every $(x, y) \in A$ with $x<a$. Then any extension of $F$ to $A \times \mathbb{R}$ enjoys the same property.

Therefore if $F: N \rightarrow \mathbb{R}^{2}$ is as above, then

1) we have not committed ourselves too much on the possible extensions of $F$ on the semispace $\sigma$ of equation $x>a$ : the only condition on such an extension is being consistent with the values of $F$ on $S \cap \pi$;

2 ) once $F$ has been extended to some $\bar{F}$ defined also on some closed subset $M$ of $\sigma, \bar{F}$ can be further extended to $A \times \mathbb{R}$ and still preserve the property that all solutions with initial condition on $S$ are trapped in $S$ until they reach $\pi$.

Similarly, if $A \subseteq \mathbb{R} \times(-\infty, b]$, a set of the form

$$
\{(x, y, z)|(x, y) \in A \&| z-G(x, y) \mid \leq \eta \cdot(b-y)\},
$$

for some $\eta>0$, is a pinched neighborhood of $S$ along the plane of equation $y=b$. 


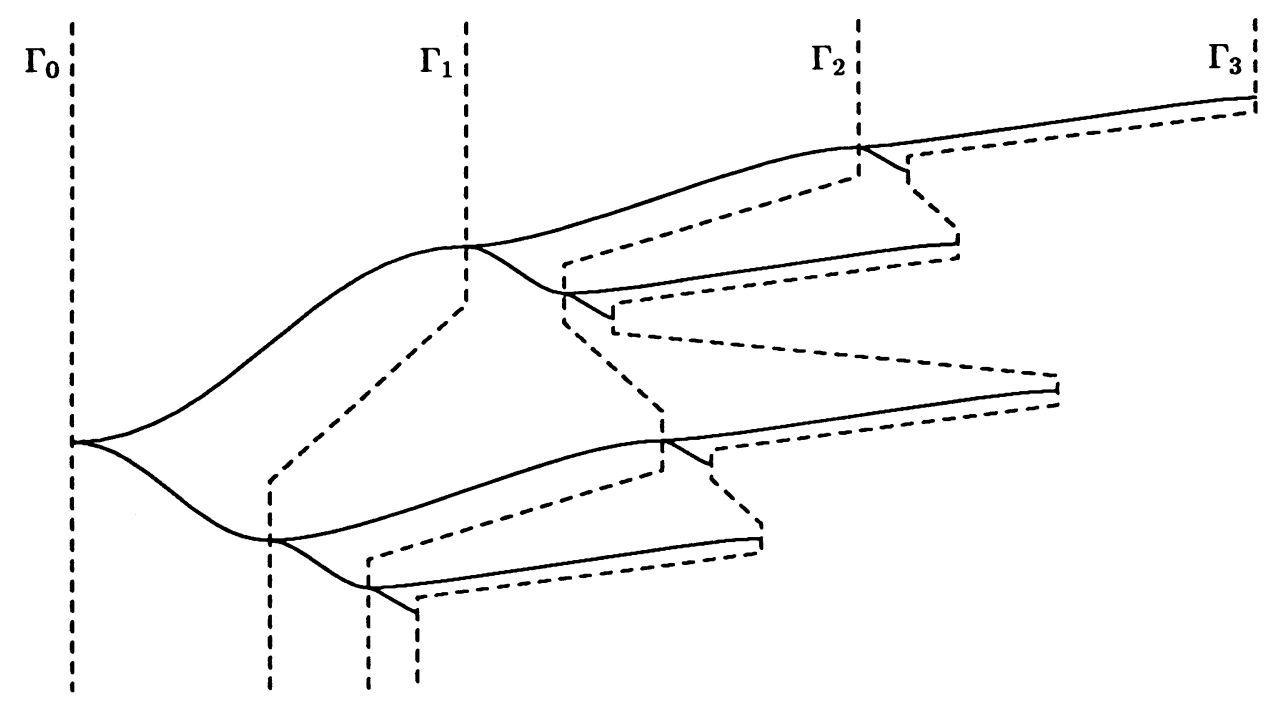

FIGURE 5 .

A similar idea is behind the following pinched neighborhood of the graph of a continuous function $\varphi$ with values in $\mathbb{R}^{2}$ and domain included in $(-\infty, a]$ :

$$
\left\{(x, y, z) \mid \sqrt{\left(y-\varphi^{\mathbf{1}}(x)\right)^{2}+\left(z-\varphi^{\mathbf{2}}(x)\right)^{2}} \leq \eta \cdot(a-x)\right\} .
$$

Here we take a closed tubular neighborhood of the graph of $\varphi$ and squeeze it as we approach the plane of equation $x=a$.

For every $n$ fix $\delta_{n}>0$ with $\delta_{n}<\frac{1}{4} \min \left\{\left|y_{s}-y_{t}\right| \mid \operatorname{lh}(s)=\operatorname{lh}(t)=n \& s \neq t\right\}$. Let $\Gamma_{n}$ be the polygonal in the $(x, y)$-plane which connects in lexicographic order the various $\left(x_{s}, y_{s}\right)$ with $\operatorname{lh}(s)=n$ and is vertical above and below each $\left(x_{s}, y_{s}\right)$ for a segment of length $2 \delta_{n}$. Extend $\Gamma_{n}$ below $\left(x_{0^{n}}, y_{0^{n}}\right)$ and above $\left(x_{1^{n}}, y_{1^{n}}\right)$ vertically. Figure 5 represents the $\Gamma_{n}$ 's with $n \leq 3$ (these are the dashed curves) together with the graphs of the $\varphi_{s}^{\mathbf{1}}$ 's for $\operatorname{lh}(s) \leq 3$. Let $H_{n}$ be the closed set in the $(x, y)$-plane bounded on the right by $\Gamma_{n}$; let also $H_{-1}=(-\infty,-1] \times \mathbb{R}$. For $n=-1,0,1, \ldots$ let

$$
B_{n}=\left(H_{n} \times \mathbb{R}\right) \cup\left(\mathbb{R}^{2} \times(-\infty, n-1]\right) \subset \mathbb{R}^{3} .
$$

Each $B_{n}$ is closed, $B_{n} \subset B_{n+1}$, and each compact subset of $\mathbb{R}^{3}$ is contained in some $B_{n}$; hence $\bigcup_{n} B_{n}=\mathbb{R}^{3}$. Then

$$
\partial\left(B_{n}\right)=\left\{(x, y, z) \mid\left[(x, y) \in \Gamma_{n} \& z \geq n-1\right] \vee\left[z=n-1 \&(x, y) \notin H_{n}\right]\right\}
$$

and $B_{n}$ is the region of $\mathbb{R}^{3}$ bounded above and on the right by $\partial\left(B_{n}\right)$. In particular $B_{-1}$ is delimited by $x=-1$ and $z=-2$, and $B_{0}$ by $x=0$ and $z=-1$. In figure 6 $\partial\left(B_{1}\right)$ is pictured: $B_{1}$ is the region of space below and to the left of it.

It is immediate to check that for any $s \in 2^{<\mathbb{N}}$

$$
\begin{aligned}
& \operatorname{lh}(s) \leq n \Longleftrightarrow \operatorname{graph}\left(\varphi_{s}\right) \subset B_{n} \quad \text { and } \\
& \operatorname{lh}(s)=n \Longleftrightarrow P_{s} \in \partial\left(B_{n}\right) .
\end{aligned}
$$

$F_{T}$ is defined by stages: at stage $n$ we define $F_{T} \uparrow A_{n}$, where $A_{n} \subset \mathbb{R}^{3}$ depends on $T$, so that: 


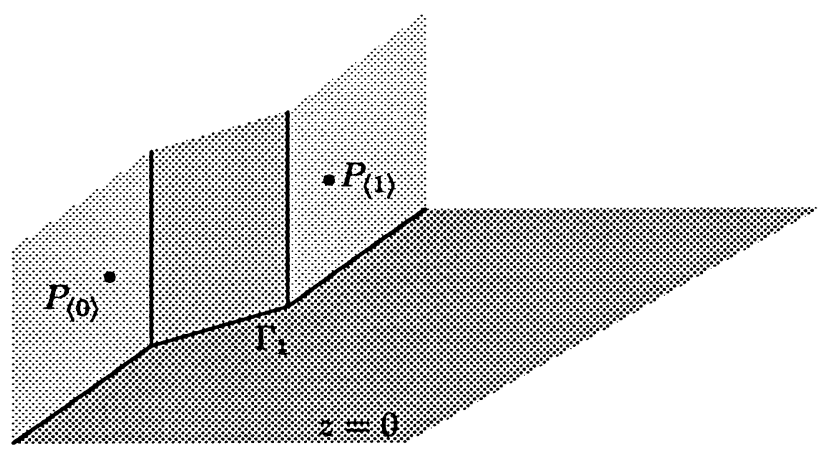

FiguRE 6.

I. $A_{n}$ is closed, $B_{n-1} \subset A_{n} \subset B_{n}$ (and hence $A_{n} \subset A_{n+1}$ ), and $F_{T} \uparrow A_{n}$ is continuous;

II. $C \cap B_{n} \subseteq A_{n}$ and $F_{T}$ satisfies (*) above; i.e. for every $s \in T\left\lceil n\right.$, graph $\left(\varphi_{s}\right) \subseteq$ $A_{n}$ and $F_{T}\left(x, \varphi_{s}(x)\right)=\varphi_{s}{ }^{\prime}(x)$ for every $x \in \operatorname{dom}\left(\varphi_{s}\right)$;

III. if $\varphi$ is a solution of $\left(F_{T} \backslash A_{n}, 0,0,0\right)$ and $\operatorname{graph}(\varphi) \cap \partial\left(A_{n}\right)=\emptyset$, then $\operatorname{dom}(\varphi)=\left(-\infty, a^{*}\right)$ and $\lim _{x \rightarrow a^{*}} \varphi(x)=\left(b^{*},+\infty\right)$ for some $\left(a^{*}, b^{*}\right)$ in the interior of $H_{n}$; in other words $\varphi$ dies to the left of $\Gamma_{n}$;

IV. if $\varphi$ is a solution of $\left(F_{T} \uparrow A_{n}, 0,0,0\right)$ and $\operatorname{graph}(\varphi) \cap \partial\left(A_{n}\right) \neq \emptyset$, then $\operatorname{graph}(\varphi) \cap \partial\left(A_{n}\right)=\left\{P_{s}\right\}$ for some $s \in T[n]$, and $P_{s \uparrow i} \in \operatorname{graph}(\varphi)$ for all $i \leq n$;

V. $A_{n}$ and $F_{T} \uparrow A_{n}$ depend only on $T\lceil n$;

VI. $A_{n} \cap \partial\left(B_{n}\right)=\left\{P_{s} \mid s \in T[n]\right\}$, so that by 【, $F_{T}\left(P_{s}\right)=\varphi_{s}{ }^{\prime}\left(x_{s}\right)$, for every $s \in T[n]$.

By 【. $F_{T}$ will be continuous and defined on $\mathbb{R}^{3}$, and by $\llbracket$ every $\varphi_{\alpha}$, with $\alpha \in[T]$, will be a solution of $\left(F_{T}, 0,0,0\right)$, so 国) holds. Suppose $\varphi$ is a global solution of $\left(F_{T}, 0,0,0\right)$. Then III implies that $\operatorname{graph}(\varphi)$ intersects each $\partial\left(A_{n}\right)$; so by IV for every $n$ there exists a unique $s_{n} \in T[n]$ such that $P_{s_{n} \backslash i} \in \operatorname{graph}(\varphi)$ for every $i \leq n$. By uniqueness $s_{n} \subset s_{n+1}$; hence $\varphi\left(x_{\alpha \nmid n}\right)=\varphi_{\alpha}\left(x_{\alpha \nmid n}\right)$ where $\alpha=\bigcup s_{n} \in[T]$. As $\varphi$ is global, $\varphi_{\alpha}$ must be global; hence $\alpha$ has infinitely many 1's. Therefore (B) holds. By $\nabla$ the map $T \mapsto F_{T}$ is continuous. Thus if our construction satisfies [V] the proof will be complete.

At stage 0 set

$$
A_{0}=\left\{(x, y, z) \mid(x \leq 0 \vee z \leq 0) \&\left(x>-1 \& z>-1 \Longrightarrow y^{2}+z^{2} \leq x^{2}\right)\right\}
$$

( $A_{0}$ consists of $B_{-1}$ together with the part lying in the semispace $x \leq 0$ of a circular cone with vertex $(0,0,0)$ and axis the $x$-axis), and let $F_{T} \uparrow A_{0}=(0,0)$. Notice that conditions \VI are satisfied.

Now suppose we have completed stage $n$. Fix $\eta=\eta_{n} \in\left(0, \delta_{n}\right]$, and for each $s \in T[n]$ and $i \in\{0,1\}$ such that $s^{\wedge}\langle i\rangle \in T$ let $D_{s, i}$ be the pinched neighborhood of the graph of $\varphi_{s<\langle i\rangle}$ defined by

$$
\begin{aligned}
\left\{(x, y, z) \mid x_{s} \leq x \leq\right. & x_{s^{-}\langle i\rangle} \\
& \left.\& \sqrt{\left(y-\varphi_{s \frown\langle i\rangle}^{\mathbf{1}}(x)\right)^{2}+\left(z-\varphi_{s \frown\langle i\rangle}^{\mathbf{2}}(x)\right)^{2}} \leq \eta \cdot\left(x_{s \frown\langle i\rangle}-x\right)\right\} .
\end{aligned}
$$




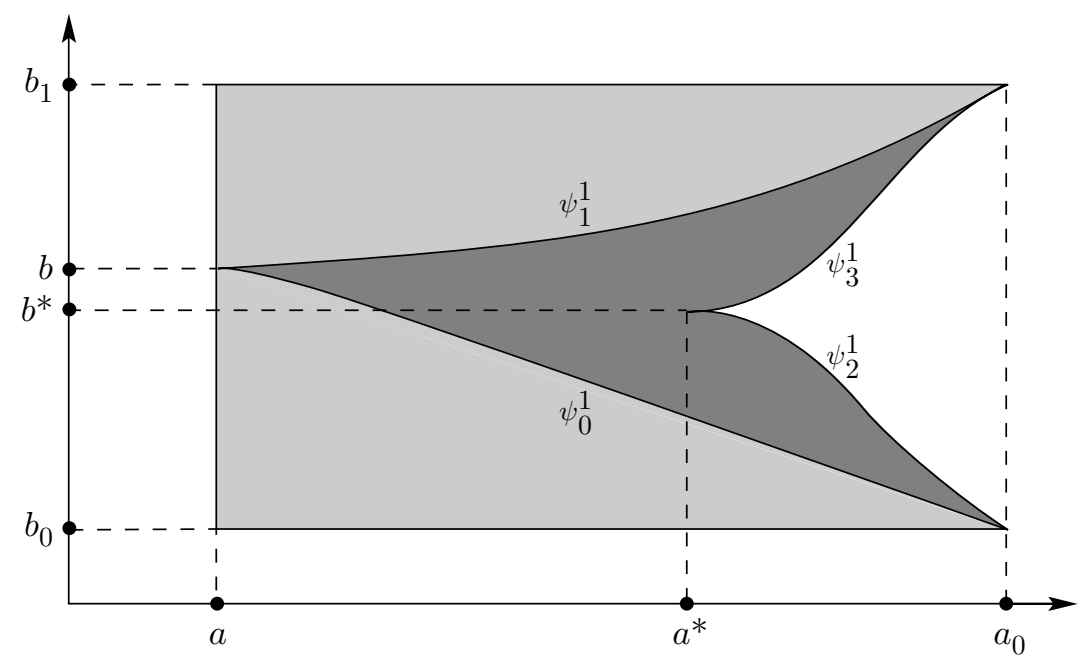

FiguRE 7.

Let $E_{s, i}$ be the projection of $D_{s, i}$ on the $(x, y)$-plane. Since $T[n]$ is finite we may assume that $\eta$ is small enough so that $E_{s, i} \cap E_{t, j}=\emptyset$ (and a fortiori $D_{s, i} \cap D_{t, j}=\emptyset$ ) whenever $s \neq t$.

For every $s \in T[n]$ at least one of $s^{\frown}\langle 0\rangle$ and $s^{\wedge}\langle 1\rangle$ belongs to $T$. If only one does, define $F_{T}$ on the relevant $D_{s, i}$ 's by setting $F_{T}(x, y, z)=\varphi_{s^{\wedge}}\langle i\rangle^{\prime}(x)$. By VI this definition is compatible with the definition of $F_{T}$ at the previous stage. Notice that $\varphi_{s<\langle i\rangle}$ is a solution of $F_{T}$ and no new bifurcation points are added by this definition because $F_{T}$ is Lipschitz (in fact: constant) in the last two variables.

If both $s^{\frown}\langle 0\rangle$ and $s^{\frown}\langle 1\rangle$ belong to $T$, the construction is much more delicate. Fix $a_{0} \in\left(x_{s}, x_{s}<\langle 0\rangle\right]$. To simplify the notation let $a=x_{s}, \psi_{0}=\varphi_{s}<\langle 0\rangle \uparrow\left[a, a_{0}\right]$, $\psi_{1}=\varphi_{s \frown\langle 1\rangle}\left\lceil\left[a, a_{0}\right],(b, c)=\left(y_{s}, n\right)=\psi_{i}(a), b_{0}=\psi_{0}^{\mathbf{1}}\left(a_{0}\right), b_{1}=\psi_{1}^{\mathbf{1}}\left(a_{0}\right)\right.$. By picking $a_{0}$ close enough to $a$ we can make sure that $\left|b-b_{0}\right|,\left|b_{1}-b\right|<\delta_{n}$, so we assume that these inequalities hold. Furthermore pick $a^{*} \in\left(a, a_{0}\right)$, and $C^{2}$ functions $\psi_{2}, \psi_{3}:\left(a^{*}, a_{0}\right] \rightarrow \mathbb{R}^{2}$ such that, if $b^{*}=\frac{1}{2}\left(\psi_{1}^{\mathbf{1}}\left(a^{*}\right)+\psi_{0}^{\mathbf{1}}\left(a^{*}\right)\right)$, we have (figure 7 represents the set-up in the $(x, y)$-plane):

1) $\psi_{2}\left(a_{0}\right)=\psi_{0}\left(a_{0}\right)$ and $\psi_{3}\left(a_{0}\right)=\psi_{1}\left(a_{0}\right)$;

2) $\psi_{2}{ }^{\prime}\left(a_{0}\right)=\psi_{0}{ }^{\prime}\left(a_{0}\right)$ and $\psi_{3}{ }^{\prime}\left(a_{0}\right)=\psi_{1}{ }^{\prime}\left(a_{0}\right)$;

3) $\psi_{0}^{\mathbf{1}}(x)<\psi_{2}^{\mathbf{1}}(x)<\psi_{3}^{\mathbf{1}}(x)<\psi_{1}^{\mathbf{1}}(x)$ for all $x \in\left(a^{*}, a_{0}\right)$;

4) $\lim _{x \rightarrow a^{*}} \psi_{2}(x)=\lim _{x \rightarrow a^{*}} \psi_{3}(x)=\left(b^{*},+\infty\right)$;

5) $\lim _{x \rightarrow a^{*}} \psi_{2}{ }^{\prime}(x)=\lim _{x \rightarrow a^{*}} \psi_{3}{ }^{\prime}(x)=\left(\frac{1}{2}\left(\left(\psi_{1}^{\mathbf{1}}\right)^{\prime}\left(a^{*}\right)+\left(\psi_{0}^{\mathbf{1}}\right)^{\prime}\left(a^{*}\right)\right),+\infty\right)$;

6) $\lim _{x \rightarrow a^{*}} \psi_{2}^{\prime \prime}(x)=\lim _{x \rightarrow a^{*}} \psi_{3}^{\prime \prime}(x)=\left(\frac{1}{2}\left(\left(\psi_{1}^{\mathbf{1}}\right)^{\prime \prime}\left(a^{*}\right)+\left(\psi_{0}^{\mathbf{1}}\right)^{\prime \prime}\left(a^{*}\right)\right),+\infty\right)$.

Let

$$
\begin{aligned}
Q= & Q_{s}= \\
& \left\{(x, y) \in\left[a, a_{0}\right] \times\left[b_{0}, b_{1}\right] \mid x>a^{*} \Longrightarrow y \leq \psi_{2}^{\mathbf{1}}(x) \vee y \geq \psi_{3}^{\mathbf{1}}(x)\right\} \backslash\left\{\left(a^{*}, b^{*}\right)\right\}
\end{aligned}
$$

( $Q$ is the shaded area in figure 7). Notice that $Q \cap H_{n} \subseteq \Gamma_{n}$ (by our choice of $a_{0}$ ) and that $s \neq t$ implies $Q_{s} \cap Q_{t}=\emptyset$. Let $G=G_{s}: Q \rightarrow \mathbb{R}$ be a $C^{2}$ function such that:

7) $G\left(x, \psi_{i}^{\mathbf{1}}(x)\right)=\psi_{i}^{\mathbf{2}}(x)$ for $i=0, \ldots, 3$ and $x \in \operatorname{dom}\left(\psi_{i}\right)$; 
8) $\lim _{(x, y) \rightarrow\left(a^{*}, b^{*}\right)} G(x, y)=+\infty$.

Again the actual definition of $G$ is irrelevant: what matters is that such a $G$ does exist. Let $S=S_{s}=\operatorname{graph}(G) \subset \mathbb{R}^{3}$; notice that $\operatorname{graph}\left(\psi_{i}\right) \subset S$ for $i=0, \ldots, 3$ and that, notwithstanding the fact that $Q$ is not closed in $\mathbb{R}^{2}, S$ is closed in $\mathbb{R}^{3}$.

Let also $Q^{*}=Q_{s}^{*}=\left\{(x, y) \in Q \mid \psi_{0}^{\mathbf{1}}(x) \leq y \leq \psi_{1}^{\mathbf{1}}(x)\right\}\left(Q^{*}\right.$ is the darkest area in figure [7). Now let

$$
N=N_{s}=\left\{(x, y, z)|(x, y) \in Q \&| z-G(x, y) \mid \leq a_{0}-x\right\} .
$$

$N$ is a pinched neighborhood of $S$ and is closed in $\mathbb{R}^{3}$. Notice also that $N \cap B_{n}=\emptyset$ and

9) $N \cap \pi=\left\{\left(a_{0}, \varphi_{s \frown\langle 0\rangle}\left(a_{0}\right)\right),\left(a_{0}, \varphi_{s \frown\langle 1\rangle}\left(a_{0}\right)\right)\right\}$, where $\pi$ is the plane $x=a_{0}$.

Claim 6. There exists a continuous $F: N \rightarrow \mathbb{R}^{2}$ such that $\psi_{0}$ and $\psi_{1}$ are solutions of the Cauchy problem $(F, a, b, c)$, and if $\zeta$ is any other solution, then either:

i) $\operatorname{dom}(\zeta)=\left[a, a_{0}\right]$ and $\zeta\left(a_{0}\right)=\psi_{i}\left(a_{0}\right)$ for some $i=0,1$, or

ii) $\operatorname{dom}(\zeta)=\left[a, a^{*}\right)$ and $\lim _{x \rightarrow a^{*}} \zeta(x)=\left(b^{*},+\infty\right)$ (so that $\zeta$ dies and cannot be further extended to the right).

Proof. The plan is to cover $S$ with a one-parameter family of curves and then use these curves to define $F$. Each one of these curves will be a solution of $F$, and as $F$ will be constant along the $z$-coordinate, they will be the only solutions for Cauchy problems with initial condition on $S$.

In order to simplify the notation let $f_{i}=\psi_{i}^{1}$, for $i=0, \ldots, 3$. Let also $f_{4}$ : $\left[a, a^{*}\right] \rightarrow \mathbb{R}$ be defined by

$$
f_{4}(x)=\frac{f_{0}(x)+f_{1}(x)}{2} .
$$

Moreover denote by $\hat{f}_{2}$, resp. $\hat{f}_{3}$, the function defined on $\left[a, a_{0}\right]$ obtained by joining $f_{2}$, resp. $f_{3}$, with $f_{4}$. Notice that $\hat{f}_{2}$ and $\hat{f}_{3}$ are both $C^{2}$. We now define a oneparameter family of curves $g$ sweeping $Q$ so that for every $(x, y) \in \hat{Q}=Q \backslash$ $\left\{(a, b),\left(a_{0}, b_{0}\right),\left(a_{0}, b_{1}\right)\right\}$ there will be a unique $g=g_{(x, y)}$ such that $(x, y) \in \operatorname{graph}(g)$ : if $(x, y)$ is between $f_{0}$ and $\hat{f}_{2}$ or $\hat{f}_{3}$ and $f_{1}$, we take a convex linear combination; while if $(x, y)$ is below $f_{0}$ or above $f_{1}$, we simply translate these functions. Notice that this implies that if $(x, y) \in\left\{(a, b),\left(a_{0}, b_{0}\right),\left(a_{0}, b_{1}\right)\right\}$, then there is a continuum of $g$ 's such that $(x, y) \in \operatorname{graph}(g)$. We state the definition of $g$ precisely. Fix $(x, y) \in \hat{Q}$ and define

$$
g_{(x, y)}= \begin{cases}f_{0}-\left(f_{0}(x)-y\right) & \text { if } y \leq f_{0}(x) ; \\ \rho f_{0}+(1-\rho) \hat{f}_{2} & \text { if } f_{0}(x)<y<\hat{f}_{2}(x) \text { and } y=\rho f_{0}(x)+(1-\rho) \hat{f}_{2}(x) ; \\ f_{i} & \text { if } y=f_{i}(x) \text { for } i=2,3,4 ; \\ \rho \hat{f}_{3}+(1-\rho) f_{1} & \text { if } \hat{f}_{3}(x)<y<f_{1}(x) \text { and } y=\rho \hat{f}_{3}(x)+(1-\rho) f_{1}(x) ; \\ f_{1}+\left(y-f_{1}(x)\right) & \text { if } f_{1}(x) \leq y .\end{cases}
$$

Thus $f_{0}, \ldots, f_{4}$ are among the $g$ 's. In figure 8 some sample $g$ 's (including all the $f_{i}$ 's) are drawn.

For $(x, y) \in \hat{Q}$ let $\xi=\xi_{(x, y)}$ be defined by

$$
\xi(t)=(g(t), G(t, g(t))),
$$

so that $\xi_{(x, y)}^{\mathbf{1}}=g_{(x, y)}$ and each curve $\xi$ lies on $S$. Therefore $\psi_{0}, \ldots, \psi_{3}$ are among the $\xi$ 's. 


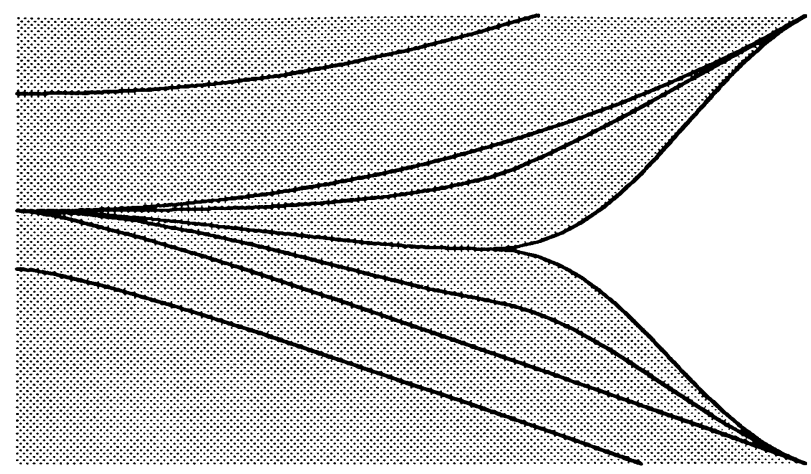

FiguRe 8.

It is easy to check that for $(x, y) \in Q^{*}$

$$
\operatorname{dom}\left(\xi_{(x, y)}\right)= \begin{cases}{\left[a, a_{0}\right]} & \text { if } y<\hat{f}_{2}(x) \text { or } y>\hat{f}_{3}(x) \\ {\left[a, a^{*}\right)} & \text { if } x<a^{*} \text { and } y=f_{4}(x) \\ \left(a^{*}, a_{0}\right] & \text { if } x>a^{*}, \text { and } y=\hat{f}_{2}(x) \text { or } y=\hat{f}_{3}(x) .\end{cases}
$$

We want the $\xi$ 's to be the solutions of $F$. Using the definition of $g_{(x, y)}$, one may check that for $(x, y) \in \hat{Q}, g_{(x, y)}$ is differentiable and that the map $\hat{Q} \rightarrow \mathbb{R},(x, y) \mapsto$ $g_{(x, y)}{ }^{\prime}(x)$, is continuous and so is the map $\hat{Q} \rightarrow \mathbb{R}^{2},(x, y) \mapsto \xi_{(x, y)}{ }^{\prime}(x)$. Let $F$ : $N \rightarrow \mathbb{R}^{2}$ be defined by

$$
F(x, y, z)= \begin{cases}\xi_{(x, y)}{ }^{\prime}(x) & \text { if }(x, y) \in \hat{Q} ; \\ \psi_{0}{ }^{\prime}(a)=\psi_{1}{ }^{\prime}(a) & \text { if }(x, y)=(a, b) ; \\ \psi_{0}{ }^{\prime}\left(a_{0}\right)=\psi_{2}{ }^{\prime}\left(a_{i}\right) & \text { if }(x, y)=\left(a_{0}, b_{0}\right) ; \\ \psi_{1}{ }^{\prime}\left(a_{0}\right)=\psi_{3}{ }^{\prime}\left(a_{i}\right) & \text { if }(x, y)=\left(a_{1}, b_{0}\right) .\end{cases}
$$

It is easy to check that $F$ is continuous. In fact $F$ is locally Lipschitz in the last two variables on $\hat{N}=N \cap(\hat{Q} \times \mathbb{R})$; hence there are no bifurcation points in the interior of $\hat{N}$. As $F$ is independent of $z$, if $\varphi$ is a solution of $(F, x, y, z)$ and $(x, y, z+\varepsilon) \in N$, then $t \mapsto\left(\varphi^{\mathbf{1}}(t), \varphi^{\mathbf{2}}(t)+\varepsilon\right)$ is a solution of $(F, x, y, z+\varepsilon)$. This easily implies that $\operatorname{graph}(\zeta) \subset S$ and $\operatorname{graph}\left(\zeta^{\mathbf{1}}\right) \subset Q^{*}$ for every solution $\zeta$ of $(F, a, b, c)$. By uniqueness in $\hat{N}$, such a $\zeta$ must be of the form $\xi_{(x, y)}$. Since all the $\xi$ 's such that $\xi(a)=(b, c)$ satisfy either i) or ii) of the statement, the proof is complete.

Claim [6 gives us the definition of $F_{T}$ on $N_{s}$ : again by VI this definition is compatible with the definition of $F_{T}$ at the previous stage. For $i=0,1$ let

$$
\hat{D}_{s, i}=D_{s, i} \cap\left(\left[a_{0}, x_{s}\langle i\rangle\right] \times \mathbb{R}^{2}\right)
$$

be the portion of $D_{s, i}$ lying in the semispace $x \geq a_{0}$. Define $F_{T}$ on $\hat{D}_{s, i}$ by letting $F_{T}(x, y, z)=\varphi_{s^{\sim}}\langle i\rangle^{\prime}(x)$, so that $F_{T}$ is Lipschitz (in fact: constant) in the last two variables. Therefore $F_{T}$ will not have bifurcation points inside $\hat{D}_{s, i}$. Notice that there is no conflict in the way $F_{T}$ is defined: as $N_{s} \cap \hat{D}_{s, i}=\left\{\left(a_{0}, \varphi_{s} \frown\langle i\rangle\left(a_{0}\right)\right)\right\}$, and-if $\eta$ has been chosen small enough- $\hat{D}_{s, 0} \cap \hat{D}_{s, 1}=\emptyset$. 
Consider the finitely many closed sets (of the form $N_{s}, D_{s, i}$ and $\hat{D}_{s, i}$ ) on which $F_{T}$ has just been defined: let $\hat{A}_{n+1}$ be the union of these sets and let $A_{n+1}=B_{n} \cup \hat{A}_{n+1}$. As $\hat{A}_{n+1}$ is closed, so is $A_{n+1}$. Now extend $F_{T}$ to $A_{n}$ using Tietze's theorem.

It is easy to check that IIVI hold, and this concludes the construction and the proof of theorem 1 .

A few remarks on this proof. It is important that the definition of $F_{T}$ on $B_{n}$ is delayed until $F_{T}$ has been prescribed on $\hat{A}_{n+1}$; otherwise we would be in trouble when defining it on the components of $\hat{A}_{n+1}$ which share points with $\partial\left(B_{n}\right)$.

As $\mathcal{G}^{1}$ is $\boldsymbol{\Sigma}_{4}^{0}$ (theorem 1 ) this proof cannot work in one dimension, so it is natural to look for what goes wrong. Suppose we try to realize the trees $T$ as closed subsets $C_{T}$ of $\mathbb{R}^{2}$ representing solutions of a differential equation, and such that branches with infinitely many 1 's correspond to global solutions. Since a solution of a differential equation in the plane is not global if and only if it has a vertical asymptote, the branches of $C_{T}$ would have to intersect. But any two solutions which intersect must have the same slope at the intersection point; hence we could switch from one solution to the other, undermining the general strategy of the proof. Obviously this would not be a problem if two solutions could intersect with different slopes: this can happen with second order differential equations and will be exploited in section 7

As the reader might have noticed, there is a simpler construction showing $\mathrm{S} \leq_{\mathrm{W}}$ $\mathcal{G}$, that now we briefly sketch. In the proof above whenever $P_{s}$ is a bifurcation point (that is $s^{\frown}\langle 0\rangle, s^{\frown}\langle 1\rangle \in T$ ), we make sure that every solution $\varphi$ going through $P_{s}$ either dies because of a vertical asymptote or else merges into $\varphi_{s}\langle 0\rangle$ or $\varphi_{s}\langle 1\rangle$. We could be less restrictive about these $\varphi$ 's by forcing them to lie on some prescribed surface $\hat{S}_{T}$ containing $C_{T}$. For the construction to succeed $\hat{S}_{T}$ must be such that none of the new $\varphi$ 's will be global unless one of the $\varphi_{\alpha}$ 's with $\alpha \in[T]$ is. The reader can visualize $\hat{S}_{T}$ by thinking of a film-soap surface passing through $C_{T}$. It is not hard to define $F_{T}$ in a neighborhood of $\hat{S}_{T}$ so that no $\varphi$ can escape $\hat{S}_{T}$. This simpler approach bypasses several technicalities (e.g. pinched neighborhoods) of the present proof. On the other hand, it does not lead to the constructions for $\mathcal{S G}$ and $\tilde{\mathcal{G}}$, where the idea of "kill one solution and merge the others" appears to be crucial. As the proofs of theorems 3 and 7 are already quite involved, we preferred to exposit the basic tricks used in sections $[$ and 7 in the simpler context of the proof of theorem 1 .

\section{6. $\mathcal{S G}$ IS $\Pi_{2}^{1}$-HARD FOR $n=2$}

We will show $\mathrm{P} \leq_{\mathrm{W}} \mathcal{S G}$ : by lemma 3 this suffices to complete the proof and establish theorem 7 . We begin by describing the strategy of the proof.

First of all a continuous function $H:\left\{(x, y, z) \in \mathbb{R}^{3} \mid x \leq 0 \vee z \leq 0\right\} \rightarrow \mathbb{R}^{2}$ is defined such that, for any continuous extension of $H$ to $\mathbb{R}^{3}$ (still denoted by $H$ ), we have $(H, 0,0,0) \in \mathcal{G}$ and

$$
(H,-3,0,0) \in \mathcal{S G} \Longleftrightarrow \exists m \forall \alpha \in 2^{\mathbb{N}}\left(H, 0,0, \mathbf{c}_{0^{m} \frown \alpha}\right) \in \mathcal{G} .
$$

(Recall the notation for the elements of the Cantor set $\mathbf{C}$, introduced in $\S 4$.) Thus the problem of verifying the globality for every initial condition in some neighborhood of $(-3,0,0)$ boils down to verifying globality for initial conditions in some interval of the Cantor space on the $z$-axis. 
Next we will define a continuous map $\mathrm{R} \rightarrow \mathbf{E q}, T \mapsto H_{T}$, such that for every $T$, $H_{T}$ extends $H$ and is such that for every $\alpha \in 2^{\mathbb{N}}$ with $\alpha \neq \overline{0}$

$$
\left(H_{T}, 0,0, \mathbf{c}_{\alpha}\right) \in \mathcal{G} \Longleftrightarrow T(\alpha) \in \mathrm{S} .
$$

To see that (11) and (2) suffice to complete the proof, recall the definition of $\tilde{T}$ and observe that for every $T \in \mathrm{R}$

$$
\begin{aligned}
T \in \mathrm{P} & \Longleftrightarrow \exists m \forall \alpha \neq \overline{0} \tilde{T}\left(0^{m \frown}\right) \in \mathrm{S} & & \text { by lemma } 4 \\
& \Longleftrightarrow \exists m \forall \alpha \neq \overline{0}\left(H_{\tilde{T}}, 0,0, \mathbf{c}_{0^{m} \sim \alpha}\right) \in \mathcal{G} & & \text { by (2) } \\
& \Longleftrightarrow \exists m \forall \alpha\left(H_{\tilde{T}}, 0,0, \mathbf{c}_{0^{m}-\alpha}\right) \in \mathcal{G} & & \text { because } \forall T\left(H_{T}, 0,0,0\right) \in \mathcal{G} \\
& \Longleftrightarrow\left(H_{\tilde{T}},-3,0,0\right) \in \mathcal{S G} & & \text { by (1). }
\end{aligned}
$$

Since the map $\mathrm{R} \rightarrow \mathbf{C p}, T \mapsto\left(H_{\tilde{T}},-3,0,0\right)$, is continuous, $\mathrm{P} \leq_{\mathrm{W}} \mathcal{S G}$.

We now define $H$ satisfying (1). $(H,-3,0,0) \in \mathcal{S G}$ requires globality to hold for initial problems belonging to a three-dimensional subset (an open ball) of $\mathbb{R}^{3}$. Our first goal is to reduce this to a one-dimensional subset: a subset of a line parallel to the $z$-axis. This is accomplished in the interval $(-\infty,-1]$ by the same technique used in the proof of theorem [6 in section 2 (figure 1 describes also our situation on any plane parallel to the $(x, y)$-plane). Fix $\chi_{0}, \chi_{1}:(-\infty,-1] \rightarrow \mathbb{R}$ as in that proof, and let $h:(-\infty,-1] \times \mathbb{R} \rightarrow \mathbb{R}$ be the function we were calling $G$. Notice that $h(-1, y)=0$. Define $H$ on $\{(x, y, z) \mid x \leq-1\}$ by $H(x, y, z)=(h(x, y), 0)$. The properties of $h$ imply that if $\varphi$ is a solution of $(H, a, b, c)$ with $a \in(-\infty,-2]$ and $b \in[-1,1]$, then $\varphi(-1)=(0, c)$. Keeping this in mind, one may immediately check that for any continuous extension of $H$ to $\mathbb{R}^{3}$ (which we still denote by $H$ ), we have

$$
(H,-3,0,0) \in \mathcal{S G} \Longleftrightarrow \exists \varepsilon>0 \forall c(|c|<\varepsilon \Longrightarrow(H,-1,0, c) \in \mathcal{G}) .
$$

In the interval $[-1,0]$ the one-dimensional subset $\{(-1,0, z)|| z \mid \leq 1\}$ will be reduced to the zero-dimensional subset $\{(0,0, c) \mid c \in \mathbf{C}\}$. We will use the following lemma, whose proof is postponed to section 8

Lemma 7. Let $E=\{(x, z)|-1 \leq x \leq 0 \&| z \mid \leq 1\}$. There exists a continuous $g: E \rightarrow \mathbb{R}$ such that:

A) for every $\alpha \in 2^{\mathbb{N}}$ and every $z \in\left[0, \mathbf{c}_{\alpha}\right]$ the one-dimensional Cauchy problem $(g,-1, z)$ has a solution $\theta$ such that for some $\beta \in 2^{\mathbb{N}}, \theta(0)=\mathbf{c}_{\beta}$ and $\mathbf{c}_{\beta} \leq \mathbf{c}_{\alpha}$ (which is equivalent to $\beta \leq_{\operatorname{lex}} \alpha$ );

B) for every $\alpha \in 2^{\mathbb{N}},\left(g,-1, \mathbf{c}_{\alpha}\right)$ has a unique solution $\theta$ and $\theta(0)=\mathbf{c}_{\alpha}$;

C) for every $z \in[-1,0),(g,-1, z)$ has a unique solution $\theta$ and $\theta(0)=0$;

D) $g(-1, z)=g(0, z)=0$ for every $z$.

Let $g$ be as in the lemma, and define $H(x, y, z)=(0, g(x, z))$ whenever $(x, z) \in E$. The conditions satisfied by $g$ imply that:

$\left.\mathrm{A}^{\prime}\right)$ for every $\alpha \in 2^{\mathbb{N}}$ and every $z \in\left[0, \mathbf{c}_{\alpha}\right]$ the two-dimensional Cauchy problem $(H,-1,0, z)$ has a solution $\varphi$ such that for some $\beta \in 2^{\mathbb{N}}, \varphi(0)=\left(0, \mathbf{c}_{\beta}\right)$ and $\mathbf{c}_{\beta} \leq \mathbf{c}_{\alpha}$

$\left.\mathrm{B}^{\prime}\right)$ for every $\alpha \in 2^{\mathbb{N}},\left(H,-1,0, \mathbf{c}_{\alpha}\right)$ has a unique solution $\varphi$ and $\varphi(0)=\left(0, \mathbf{c}_{\alpha}\right)$;

$\left.\mathrm{C}^{\prime}\right)$ for every $z \in[-1,0),(H,-1,0, z)$ has a unique solution $\varphi$ and $\varphi(0)=(0,0)$;

$\left.\mathrm{D}^{\prime}\right) H(0, y, z)=(0,0)$ for every $y$ and $z$. 
Therefore for any continuous extension of $H$ to $\mathbb{R}^{3}$ (which we still denote by $H$ ) and any $\varepsilon>0$, we have that $\forall z(|z|<\varepsilon \Longrightarrow(H,-1,0, z) \in \mathcal{G})$ is implied by

$$
\forall \alpha\left(\mathbf{c}_{\alpha}<3 \varepsilon \Longrightarrow\left(H, 0,0, \mathbf{c}_{\alpha}\right) \in \mathcal{G}\right) \text {. }
$$

(We are using the fact that $[\varepsilon, 3 \varepsilon$ ) intersects $\mathbf{C}$ for every $\varepsilon>0$.) Using the fact that $\mathbf{c}_{\alpha} \leq 3^{-m}$ if and only if $\alpha \uparrow m=0^{m}$, one finds that

(4) $\exists \varepsilon>0 \forall z(|z|<\varepsilon \Longrightarrow(H,-1,0, z) \in \mathcal{G}) \Longleftrightarrow \exists m \forall \alpha\left(H, 0,0, \mathbf{c}_{0^{m} \frown \alpha}\right) \in \mathcal{G}$.

Combining (3) and (4) we have that for any continuous extension of $H$ to $\mathbb{R}^{3}$,

$$
(H,-3,0,0) \in \mathcal{S G} \Longleftrightarrow \exists m \forall \alpha\left(H, 0,0, \mathbf{c}_{0^{m} \frown \alpha}\right) \in \mathcal{G},
$$

i.e. (1). Set $H(x, 0,0)=(0,0)$ for every $x>0$, and use Tietze's extension theorem to extend $H$ to $\left\{(x, y, z) \in \mathbb{R}^{3} \mid x \leq 0 \vee z \leq 0\right\}$. Clearly $(H, 0,0,0) \in \mathcal{G}$ is now guaranteed.

Now that (1) has been achieved we concentrate on (2). To this end the construction of section 5 will be used: in that section we constructed, for every $T \in \mathrm{PTr}$, $F_{T} \in \mathbf{E q}$ such that $T \in \mathrm{S} \Longleftrightarrow\left(F_{T}, 0,0,0\right) \in \mathcal{G}$. Other features of that construction that will be relevant are $F_{T}(0,0,0)=0$ and if $\varphi$ is a solution of $\left(F_{T}, 0,0,0\right)$, then $\varphi^{2}(x)>0$ for every $x>0$.

Let $Q_{T} \subseteq \mathbb{R}^{2}$ be the union of the graphs of the $\varphi_{s}^{\mathbf{1}}$ with $s \in T$ and $\operatorname{lh}(s)>0$ and of the $Q_{s}^{*}$ with $s^{\frown}\langle 0\rangle, s^{\frown}\langle 1\rangle \in T\left(Q_{s}^{*}\right.$ has been defined before claim 6] in figure 7 $Q_{s}^{*}$ is the darkest area). Let $G_{T}: Q_{T} \rightarrow \mathbb{R}$ be defined by $G_{T}\left(x, \varphi_{s}^{\mathbf{1}}(x)\right)=\varphi_{s}^{\mathbf{2}}(x)$ and $G_{T} \uparrow Q_{s}^{*}=G_{s} \uparrow Q_{s}^{*}$, where $G_{s}$ has also been defined before claim 6 Notice that $G_{T}$ is continuous, and let $S_{T}$ be the graph of $G_{T}$. We have that

$S_{T} \supseteq\left\{(x, y, z) \mid x \geq 0 \& \exists \varphi\left(\varphi\right.\right.$ is a solution of $\left.\left.\left(F_{T}, 0,0,0\right) \& \varphi(x)=(y, z)\right)\right\}$

and that $S_{T}$ is closed (even if $Q_{T}$ is not closed).

From the construction of $F_{T}$ it also follows that if $s \in T_{0} \cap T_{1}$, then $F_{T_{0}}$ and $F_{T_{1}}$ agree on the graph of $\varphi_{s}$, and if $s^{\frown}\langle 0\rangle, s^{\frown}\langle 1\rangle \in T_{0} \cap T_{1}$, also on the graph of $G_{s}$. Therefore $F_{T_{0}} \uparrow\left(S_{T_{0}} \cap S_{T_{1}}\right)=F_{T_{1}} \uparrow\left(S_{T_{0}} \cap S_{T_{1}}\right)$, for any $T_{0}, T_{1} \in \mathrm{PTr}$.

We shift along the $z$-axis this construction by defining, for a fixed $\alpha \in 2^{\mathbb{N}}$, $F_{T}^{\alpha}(x, y, z)=F\left(x, y, z-\mathbf{c}_{\alpha}\right)$ and obtaining

$$
T \in \mathrm{S} \Longleftrightarrow\left(F_{T}^{\alpha}, 0,0, \mathbf{c}_{\alpha}\right) \in \mathcal{G} .
$$

Then if $G_{T}^{\alpha}: Q_{T} \rightarrow \mathbb{R}$ is defined by $G_{T}^{\alpha}(x, y)=G_{T}(x, y)+\mathbf{c}_{\alpha}$, we have that the graph $S_{T}^{\alpha}$ of $G_{T}^{\alpha}$ is closed and satisfies

$S_{T}^{\alpha} \supseteq\left\{(x, y, z) \mid x \geq 0 \& \exists \varphi\left(\varphi\right.\right.$ is a solution of $\left.\left.\left(F_{T}^{\alpha}, 0,0, \mathbf{c}_{\alpha}\right) \& \varphi(x)=(y, z)\right)\right\}$.

The considerations made above imply that if $T_{0}, T_{1} \in \mathrm{P} \operatorname{Tr}$ and $\alpha, \beta \in 2^{\mathbb{N}}$ with $\alpha \neq \beta$, then $G_{T_{0}}^{\alpha}(x, y) \neq G_{T_{1}}^{\beta}(x, y)$ for every $(x, y) \in Q_{T_{0}} \cap Q_{T_{1}}$, i.e. $S_{T_{0}}^{\alpha} \cap S_{T_{1}}^{\beta}=\emptyset$.

Let now $T \in \mathrm{R}$ be a tree on the product $2 \times 2$ to which we need to associate $H_{T} \in \mathbf{E q}$ satisfying (2). We will make sure that for $\alpha \in 2^{\mathbb{N}}$ each solution of $\left(H_{T}, 0,0, \mathbf{c}_{\alpha}\right)$ is a solution of $F_{T(\alpha)}^{\alpha}$ and hence lies on $S_{T(\alpha)}^{\alpha}$. The naive way of doing so is to check that $\bigcup_{\alpha \in 2^{\mathbb{N}}} S_{T(\alpha)}^{\alpha}$ is closed, define $H_{T}=F_{T(\alpha)}^{\alpha}$ on $S_{T(\alpha)}^{\alpha}$ and use Tietze's extension theorem. This is not enough, because for the equivalence (5) to hold we need the appropriate definition of $F_{T}^{\alpha}$, not only on $S_{T(\alpha)}^{\alpha}$, but also in a neighborhood of it (to avoid spurious solutions which "escape" $\left.S_{T(\alpha)}^{\alpha}\right)$.

To address this problem we will exploit the fact that the map $\alpha \mapsto T(\alpha)$ has the (obvious) property that $\alpha \uparrow n=\beta \uparrow n$ implies $T(\alpha) \uparrow n=T(\beta) \uparrow n$. Thus, since up 
to stage $n$ the definition of $F_{T(\alpha)}$ depends only on $T(\alpha) \uparrow n$, we have- up to that stage - the same definition for $F_{T(\beta)}$.

Just as in the proof of section 5, $H_{T}$ is defined by stages, and suppose we have completed stage $n$ (since $H_{T} \uparrow\{(x, y, z) \mid x \leq 0 \vee z \leq 0\}=H$, there is no need to deal with stage 0 ).

For $X \subseteq \mathbb{R}^{3}$ and $\overrightarrow{\mathbf{u}}$ a vector of $\mathbb{R}^{3}, X+\overrightarrow{\mathbf{u}}=\{P+\overrightarrow{\mathbf{u}} \mid P \in X\}$ is the translate of $X$ via $\overrightarrow{\mathbf{u}}$ and $\bigcup_{a \leq \lambda \leq b} X+\lambda \overrightarrow{\mathbf{u}}$ is the region swept by continuously moving $X+a \overrightarrow{\mathbf{u}}$ to $X+b \overrightarrow{\mathbf{u}}$.

Recall that in section 5 we defined the pinched neighborhood $D_{s, i}$ of the graph of $\varphi_{s^{\frown}\langle i\rangle}$. For $(t, s) \in T[n]$ and $i, j<2$ such that $\left(t^{\frown}\langle j\rangle, s^{\frown}\langle i\rangle\right) \in T$ let

$$
\begin{aligned}
D_{s, i}^{t, j} & =\underset{\mathbf{c}_{t \frown\langle j\rangle}^{0} \leq \lambda \leq \mathbf{c}_{t^{1}\langle j\rangle}}{\bigcup} D_{s, i}+\lambda \overrightarrow{\mathbf{k}} \\
& =\left\{(x, y, z) \mid \exists\left(x, y, z^{\prime}\right) \in D_{s, i}\left(\mathbf{c}_{t^{-\langle j\rangle}}^{0} \leq z-z^{\prime} \leq \mathbf{c}_{t^{-}\langle j\rangle}^{1}\right)\right\},
\end{aligned}
$$

where $\overrightarrow{\mathbf{k}}=(0,0,1)$ is the unit vector on the $z$-axis. Recall that the definition of $D_{s, i}$, and hence that of $D_{s, i}^{t, j}$, depends on some $\eta=\eta_{n}>0$ : we may assume that $\eta$ is small enough to make sure $D_{s_{0}, i_{0}}^{t_{0}, j_{0}} \cap D_{s_{1}, i_{1}}^{t_{1}, j_{1}}=\emptyset$ whenever $\left(t_{0} \frown\left\langle j_{0}\right\rangle, s_{0}\right) \neq\left(t_{1} \frown\left\langle j_{1}\right\rangle, s_{1}\right)$.

For every $(t, s) \in T[n]$ and every $j$, at least one of $\left(t^{\frown}\langle j\rangle, s^{\frown}\langle 0\rangle\right)$ and $\left(t^{\frown}\langle j\rangle\right.$, $s^{\frown}\langle 1\rangle$ ) belongs to $T$ (because $T \in \mathrm{R}$ ). If only one does, define $H_{T}$ on the relevant $D_{s, i}^{t, j}$ by

$$
H_{T}(x, y, z)=\varphi_{s \frown\langle i\rangle}{ }^{\prime}(x) .
$$

If both $\left(t^{\frown}\langle j\rangle, s^{\frown}\langle 0\rangle\right)$ and $\left(t^{\frown}\langle j\rangle, s^{\frown}\langle 1\rangle\right)$ belong to $T$, let

$$
\begin{aligned}
& N_{s}^{t, j}=\underset{\mathbf{c}_{t^{\prime}\langle j\rangle}^{0} \leq \lambda \leq \mathbf{c}_{t^{1}\langle j\rangle}^{1}}{\bigcup} N_{s}+\lambda \overrightarrow{\mathbf{k}} \\
& =\left\{(x, y, z) \mid \mathbf{c}_{t^{-}\langle j\rangle}^{0}-\eta \cdot\left(x_{s^{\frown}\langle 0\rangle}-x\right) \leq z-G_{s}(x, y) \leq \mathbf{c}_{t^{-}\langle j\rangle}^{1}+\eta \cdot\left(x_{s^{\frown}\langle 0\rangle}-x\right)\right\} .
\end{aligned}
$$

$N_{s}^{t, j}$ is a pinched neighborhood of the "thick" surface $\bigcup_{\mathbf{c}^{0}\langle\langle j\rangle} \leq \lambda \leq \mathbf{c}_{t^{\prime} \backslash\langle j\rangle} S_{s}+\lambda \overrightarrow{\mathbf{k}}$. By choosing $\eta$ small enough (we are assuming this is the same $\eta$ used in the definition of $\left.D_{s, i}^{t, j}\right)$ we can make sure that $N_{s_{0}}^{t_{0}, j_{0}} \cap N_{s_{1}}^{t_{1}, j_{1}}=\emptyset$ and $N_{s_{0}}^{t_{0}, j_{0}} \cap D_{s_{1}, i}^{t_{1}, j_{1}}=\emptyset$ whenever $\left(t_{0} \frown\left\langle j_{0}\right\rangle, s_{0}\right) \neq\left(t_{1} \frown\left\langle j_{1}\right\rangle, s_{1}\right)$.

On $N_{s}^{t, j}$ let

$$
H_{T}(x, y, z)=F_{T(\alpha)}\left(x, y, z^{\prime}\right) \quad \text { where } \alpha \supset t^{\frown}\langle j\rangle \text { and }\left(x, y, z^{\prime}\right) \in N_{s} .
$$

The choices of $\alpha$ and $z^{\prime}$ are irrelevant: if $\alpha, \beta \supset t^{\frown}\langle j\rangle$, then $T(\alpha) \uparrow(n+1)=$

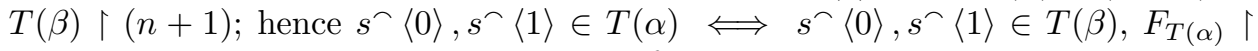
$N_{s}=F_{T(\beta)} \uparrow N_{s}$, and it is independent from the $z$-coordinate. For $i=0,1$ on $D_{s, i}^{t, j} \cap\left(\left[x_{s^{-}\langle 0\rangle}, x_{s^{\frown}\langle i\rangle}\right] \times \mathbb{R}^{2}\right)$ let

$$
H_{T}(x, y, z)=\varphi_{s-\langle i\rangle}{ }^{\prime}(x) .
$$

As in the proof of section 5, this is not in conflict with our previous definitions.

The only possible conflict between this definition and the previous definition of $H$ on $\{(x, y, z) \mid x \leq 0 \vee z \leq 0\}$ is when $n=0, t=s=\langle\rangle$ and $j=0$. In this case $D_{\langle\rangle, 0}^{\langle\rangle, 0}, D_{\langle\rangle, 1}^{\langle\rangle, 0}$ and $N_{\langle\rangle}^{\langle\rangle, 0}$ intersect the previous domain, no matter how small we pick $\eta_{0}$ (when $n>0$ surely $\eta_{n}$ will be small enough to avoid any intersections). Since 
(2) is only required of $\alpha \neq \overline{0}$, we can be a bit more liberal with the definition of $F_{T(\overline{0})}$ and allow a few more solutions spurring from the origin. So instead of $D_{\langle\rangle, i}^{\langle\rangle, 0}$ and $N_{\langle\rangle}^{\langle\rangle, 0}$ use

$$
\begin{aligned}
& \hat{D}_{\langle\rangle, i}^{\langle\rangle, 0}=\left\{(x, y, z) \in D_{\langle\rangle, i}^{\langle\rangle, 0} \mid z>\varphi_{\langle i\rangle}^{2}(x)\right\} \quad \text { and } \\
& \hat{N}_{\langle\rangle}^{\langle\rangle, 0}=\left\{(x, y, z) \in N_{\langle\rangle}^{\langle\rangle, 0} \mid z \geq G_{\langle\rangle}(x, y)\right\} .
\end{aligned}
$$

This way the only intersection between $\{(x, y, z) \mid x \leq 0 \vee z \leq 0\}$ and one of these sets is $(0,0,0)$, and both definitions prescribe $H_{T}(0,0,0)=(0,0)$.

We can now use Tietze's extension theorem to extend $H_{T}$ to $\mathbb{R}^{3}$ : by applying it stage by stage (as in section 5) we make sure that $T \mapsto H_{T}$ is continuous. By our construction we have that for $\alpha \neq \overline{0}$ each solution of $\left(H_{T}, 0,0, \mathbf{c}_{\alpha}\right)$ lies on $S_{T(\alpha)}^{\alpha}$ and hence that

$$
\left(H_{T}, 0,0, \mathbf{c}_{\alpha}\right) \in \mathcal{G} \Longleftrightarrow T(\alpha) \in \mathrm{S}
$$

i.e. (2).

$$
\text { 7. } \tilde{\mathcal{G}} \text { IS } \boldsymbol{\Sigma}_{1}^{1} \text {-HARD FOR } n=2
$$

We will define a continuous map $\mathrm{PTr} \rightarrow \widetilde{\mathbf{E q}}, T \mapsto \tilde{F}_{T}$, so that

$$
T \in \mathrm{S} \Longleftrightarrow\left(\tilde{F}_{T}, 0,0,1\right) \in \tilde{\mathcal{G}},
$$

witnessing $\mathrm{S} \leq_{\mathrm{W}} \tilde{\mathcal{G}}$.

The main idea of the proof is very similar to the one of the proof of the $\boldsymbol{\Sigma}_{1}^{1}$ hardness of $\mathcal{G}$ in section 5 , but we have less freedom than in dimension 2 . In fact we can identify a solution of a second order differential equation with its lifting (which is a function with values in $\mathbb{R}^{2}$, as are the functions considered in section 57), but not all functions $\varphi$ with values in $\mathbb{R}^{2}$ are the lifting of a $C^{2}$ function with values in $\mathbb{R}$ : liftings satisfy $\varphi^{2}=\left(\varphi^{\mathbf{1}}\right)^{\prime}$. The functions $\varphi_{\alpha}$ used in section 5 do not satisfy this condition, because $\varphi_{\alpha}^{2}$ is increasing and unbounded above, while $\varphi_{\alpha}^{\mathbf{1}}$ is decreasing on some intervals. Thus we need to define new functions (which we still denote by $\varphi_{s}$ and $\varphi_{\alpha}$ ) which represent a tree as a subset of $\mathbb{R}^{2}$ (and hence, by means of their liftings, of $\mathbb{R}^{3}$ ). We use again the $x_{s}$ 's and the $x_{\alpha}$ 's defined on page 54. We use functions whose graphs intersect (although we will make sure that their liftings do not): in fact we cannot have the third coordinate (which now represents the derivative) be unbounded above as $x$ approaches $x_{\alpha}$ without having the second coordinate also unbounded above. Every $s \in 2^{<\mathbb{N}}$ is identified with the point $P_{s}=\left(x_{s}, \operatorname{lh}(s)\right) \in \mathbb{R}^{2}$. Since for some $s$ and $t$ we have $x_{s}<x_{t}$ but $x_{s^{\wedge}\langle 1\rangle}>x_{t \prec\langle 0\rangle}$, the need for the graphs to intersect is obvious. For every $s \in 2^{<\mathbb{N}}$ with $\operatorname{lh}(s)>0$ recall again that $s^{-}=s \uparrow(\operatorname{lh}(s)-1)$, and define a family of $C^{2}$ functions $\varphi_{s}:\left[x_{s^{-}}, x_{s}\right] \rightarrow\left[\ln \left(s^{-}\right), \operatorname{lh}(s)\right]$ such that:

i) $\varphi_{s}$ is strictly increasing onto its range (so that the graph of $\varphi_{s}$ connects $P_{s^{-}}$ with $P_{s}$ );

ii) $\varphi_{s}^{\prime}\left(x_{s^{-}}\right)=\varphi_{s}^{\prime}\left(x_{s}\right)=1$;

iii) $\varphi_{s}^{\prime \prime}\left(x_{s^{-}}\right)=\varphi_{s}^{\prime \prime}\left(x_{s}\right)=0$;

iv) for every $x \in\left(x_{s}, x_{s \prec\langle 0\rangle}\right], \varphi_{s^{\prec}\langle 0\rangle^{\prime}}(x)>\varphi_{s^{\prec}\langle 1\rangle^{\prime}}(x)$ and hence $\varphi_{s \frown\langle 0\rangle}(x)>$ $\varphi_{s} \sim\langle 1\rangle(x)$

v) if $\operatorname{lh}(s)=\operatorname{lh}(t), s^{-} \neq t^{-}$and $\varphi_{s}(x)=\varphi_{t}(x)$, then $\varphi_{s}{ }^{\prime}(x) \neq \varphi_{t}^{\prime}(x)$ (this condition can be met because $s \neq t$ implies $x_{s} \neq x_{t}$ ). 


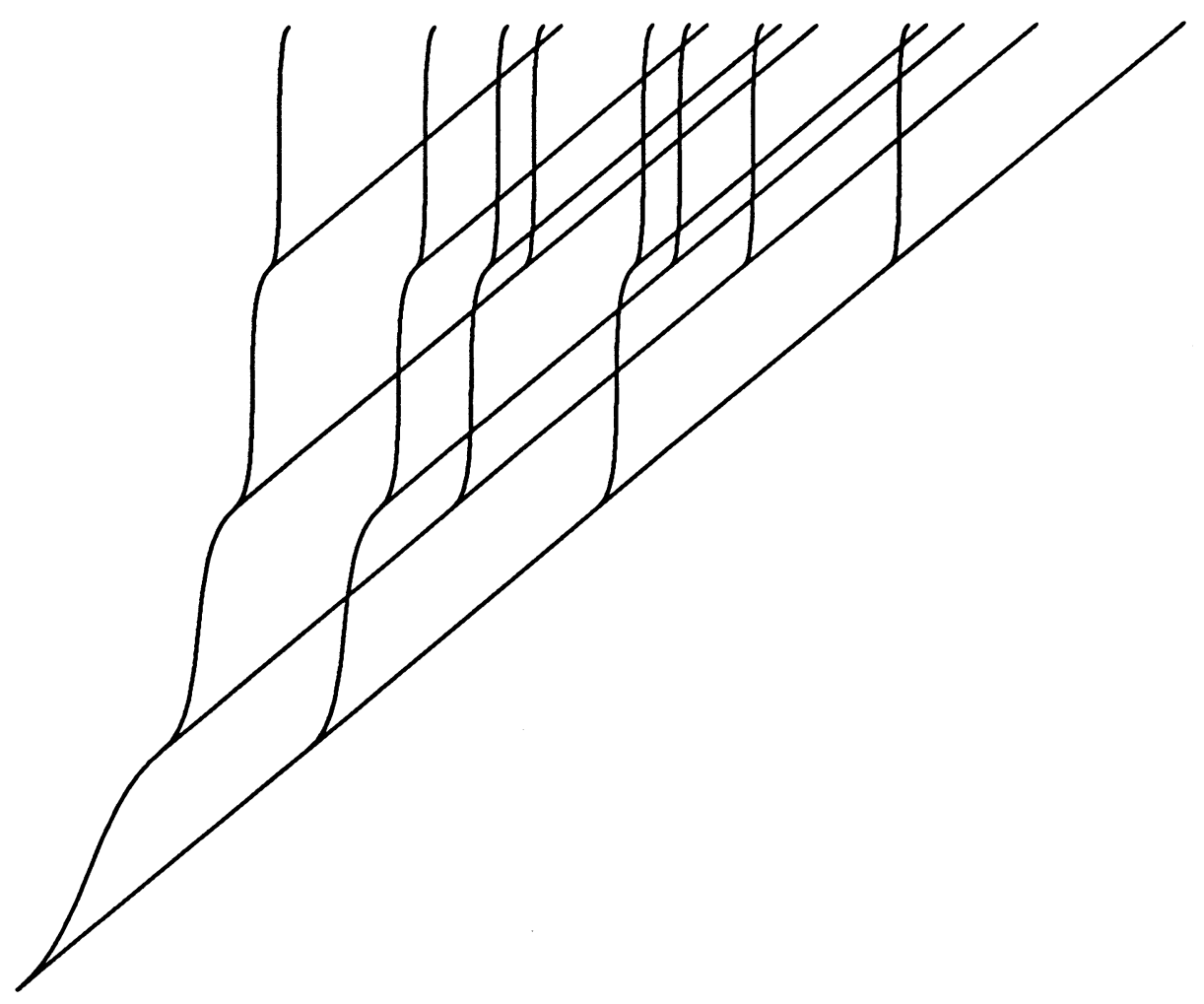

FiguRE 9.

Figure 9 represents the $\varphi_{s}$ 's for $\ln (s) \leq 4$. The actual definitions of the $\varphi_{s}$ 's are irrelevant here - the only important thing is to observe that $\varphi_{s}$ 's as above do exist. Define also $\varphi_{\langle\rangle}:(-\infty, 0] \rightarrow \mathbb{R}$ by setting $\varphi_{\langle\rangle}(x)=x$ for every $x \leq 0$.

For every $\alpha \in 2^{\mathbb{N}}$ we now define $\varphi_{\alpha}:\left(-\infty, x_{\alpha}\right) \rightarrow \mathbb{R}$ by setting

$$
\varphi_{\alpha}(x)= \begin{cases}\varphi_{\langle\rangle}(x) & \text { if } x<0 \\ \varphi_{\alpha \uparrow(n+1)}(x) & \text { if } x \in\left[x_{\alpha \nmid n}, x_{\alpha \uparrow(n+1)}\right) .\end{cases}
$$

Conditions (iii) and (iii) on the $\varphi_{s}$ 's imply that $\varphi_{\alpha}$ is $C^{2}$. Moreover we have if $n$ is such that $\alpha\left\lceil n=\beta\left\lceil n\right.\right.$ but $\alpha(n)<\beta(n)$, then $\varphi_{\alpha}(x)=$ $\varphi_{\beta}(x)$ for every $x \in\left(-\infty, x_{\alpha \mid n}\right]$ and $\varphi_{\alpha}{ }^{\prime}(x) \neq \varphi_{\beta}{ }^{\prime}(x)$ for every $x \in\left(x_{\alpha \mid n}, \min \left(x_{\alpha}, x_{\beta}\right)\right)$ such that $\varphi_{\alpha}(x)=\varphi_{\beta}(x)$.

Therefore $\varphi_{\alpha}(x)=\varphi_{\beta}(x) \& \varphi_{\alpha}{ }^{\prime}(x)=\varphi_{\beta}{ }^{\prime}(x) \Longrightarrow \varphi_{\alpha}{ }^{\prime \prime}(x)=\varphi_{\beta}{ }^{\prime \prime}(x)$.

Let

$$
C=C_{T}=\left\{(x, y, z) \mid \exists \alpha \in[T]\left(x<x_{\alpha} \& \varphi_{\alpha}(x)=y \& \varphi_{\alpha}^{\prime}(x)=z\right)\right\}
$$

be the union of the graphs of the liftings of the $\varphi_{\alpha}$ 's with $\alpha \in[T]$, and let $\tilde{F}_{T} \uparrow C$ be

$$
\begin{aligned}
\tilde{F}_{T}(x, y, z)=\varphi_{\alpha}{ }^{\prime \prime}(x) & \text { for some (every) } \alpha \in[T] \\
& \text { such that } \varphi_{\alpha}(x)=y \text { and } \varphi_{\alpha}{ }^{\prime}(x)=z .
\end{aligned}
$$


The only problem left is to define $\tilde{F}_{T}$ on $\mathbb{R}^{3} \backslash C$ in such a way that $\left(\tilde{F}_{T}, 0,0,1\right) \in \mathcal{G}$ just in case some $\varphi_{\alpha}$ is global. Again we would like the $\varphi_{\alpha}$ 's, with $\alpha \in[T]$, to be the only solutions of $\left(\tilde{F}_{T}, 0,0,1\right)$ : as this cannot be literally true, whenever $s^{\frown}\langle 0\rangle, s^{\frown}\langle 1\rangle \in T$ we will kill one solution passing through $P_{s}$ and merge the others into $\varphi_{s}\langle 0\rangle$ or $\varphi_{s^{\prec}\langle 1\rangle}$. $\tilde{F}_{T}$ will satisfy the following conditions:

a) if $\alpha \in[T]$ then $\varphi_{\alpha}$ is a solution of $\left(\tilde{F}_{T}, 0,0,1\right)$;

b) if $\varphi$ is a global solution of $\left(\tilde{F}_{T}, 0,0,1\right)$, then there exists a unique $\alpha \in[T]$ with infinitely many 1's such that $\varphi\left(x_{\alpha \nmid n}\right)=\varphi_{\alpha}\left(x_{\alpha \nmid n}\right)$ for every $n$.

Therefore $\left(\tilde{F}_{T}, 0,0,1\right) \in \tilde{\mathcal{G}}$ if and only if $T \in \mathrm{S}$. Thus the proof will be complete, provided that the map $T \mapsto \tilde{F}_{T}$ is continuous.

For $n=-1,0,1, \ldots$ let $B_{n}=\mathbb{R} \times(-\infty, n] \times \mathbb{R}$, so that each compact subset of $\mathbb{R}^{3}$ is contained in some $B_{n}$. It is immediate to check that $\operatorname{lh}(s) \leq n$ if and only if the graph of the lifting of $\varphi_{s}$ is included in $B_{n}$, for any $s \in 2^{<\mathbb{N}}$.

$\tilde{F}_{T}$ is defined by stages: at stage $n$ we define $\tilde{F}_{T} \uparrow A_{n}$, where $A_{n} \subseteq \mathbb{R}^{3}$ depends on $T$, so that:

I. $A_{n}$ is closed, $B_{n-1} \subset A_{n} \subset B_{n}$ (and hence $A_{n} \subset A_{n+1}$ ), and $\tilde{F}_{T} \uparrow A_{n}$ is continuous;

II. $C \cap B_{n} \subseteq A_{n}$ and $F_{T}$ satisfies (*) above, i.e. for every $s \in T\lceil n$, the graph of the lifting of $\varphi_{s}$ is contained in $A_{n}$ and $\tilde{F}_{T}\left(x, \varphi_{s}(x), \varphi_{s}{ }^{\prime}(x)\right)=\varphi_{s}{ }^{\prime \prime}(x)$ for every $x \in \operatorname{dom}\left(\varphi_{s}\right)$;

III. if $\varphi$ is a solution of $\left(\tilde{F}_{T}\left\lceil A_{n}, 0,0,1\right)\right.$ and $\left(x, \varphi(x), \varphi^{\prime}(x)\right) \notin \partial\left(A_{n}\right)$ for every $x \in \operatorname{dom}(\varphi)$, then $\operatorname{dom}(\varphi)=\left(-\infty, a^{*}\right), \lim _{x \rightarrow a^{*}} \varphi(x)=b^{*}, \lim _{x \rightarrow a^{*}} \varphi(x)^{\prime}=$ $+\infty$, for some $\left(a^{*}, b^{*}\right) \in \mathbb{R} \times(-\infty, n)$; in other words $\varphi$ dies without reaching the line $y=n$;

IV. if $\varphi$ is a solution of $\left(\tilde{F}_{T}\left\lceil A_{n}, 0,0,1\right)\right.$ and $\left(x, \varphi(x), \varphi^{\prime}(x)\right) \in \partial\left(A_{n}\right)$ for some $x \in \operatorname{dom}(\varphi)$, then $x=x_{s}, \varphi\left(x_{s}\right)=n$ and $\varphi_{s}{ }^{\prime}\left(x_{s}\right)=1$ for some $s \in T[n]$; moreover $\varphi\left(x_{s \uparrow i}\right)=i$ and $\varphi^{\prime}\left(x_{s \uparrow i}\right)=1$ for all $i \leq n$;

V. $A_{n}$ and $\tilde{F}_{T}\left\lceil A_{n}\right.$ depend only on $T\lceil n$;

VI. $A_{n} \cap \partial\left(B_{n}\right)=\left\{\left(x_{s}, n, 1\right) \mid s \in T[n]\right\}$, so that by $\amalg$, $\tilde{F}_{T}\left(x_{s}, n, 1\right)=0$, for every $s \in T[n]$.

[पVI imply the continuity of $\tilde{F}_{T}$, 国), (b) , and the continuity of $T \mapsto \tilde{F}_{T}$ exactly as the corresponding conditions for the two-dimensional case (see §5) do. Thus if our construction satisfies \पVI the proof will be complete.

At stage 0 set

$$
A_{0}=\left\{(x, y, z) \mid y \leq 0 \&\left(y>-1 \Longrightarrow x^{2}+z^{2} \leq y^{2}\right)\right\}
$$

( $A_{0}$ consists of $B_{-1}$ together with the part lying in $B_{0}$ of a circular cone with vertex $(0,0,0)$ and axis the $y$-axis $)$, and let $\tilde{F}_{T}\left\lceil A_{0}=0\right.$. Notice that conditions [VT]are satisfied.

Now suppose we have completed stage $n$. Fix $b \in(n, n+1)$. For each $s \in T[n]$ and $i \in\{0,1\}$ such that $s^{\frown}\langle i\rangle \in T$ let $x_{s}^{i}=\varphi_{s^{\curlyvee}\langle i\rangle}^{-1}(b)$. As $T[n]$ is finite we can choose $b$ close enough to $n$ so that for every $s, t \in T[n]$ and $i$ as above, $x_{s}<x_{t}$ implies $x_{s}^{i}<x_{t}$. Fix also $\eta=\eta_{n}>0$. For each $s$ and $i$ as above let $D_{s, i}$ be the set

$$
\begin{aligned}
& \left\{(x, y, z) \mid x_{s} \leq x \leq x_{s \frown\langle i\rangle} \& y \geq n\right. \\
& \left.\quad \& \sqrt{\left(y-\varphi_{s<\langle i\rangle}(x)\right)^{2}+\left(z-\varphi_{s \frown\langle i\rangle}{ }^{\prime}(x)\right)^{2}} \leq \eta \cdot\left(x_{s \frown\langle i\rangle}-x\right)\right\} .
\end{aligned}
$$




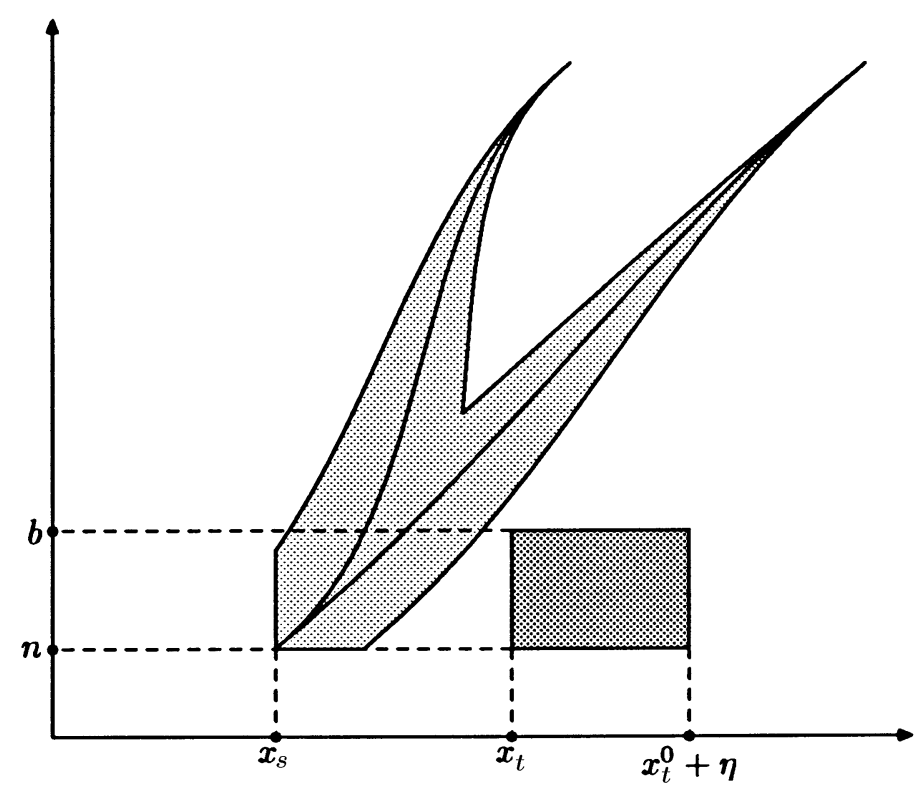

Figure 10.

$D_{s, i}$ is a pinched neighborhood of the lifting of $\varphi_{s} \sim\langle i\rangle$, and let $E_{s, i}$ be its projection on the $(x, y)$-plane. Again by the finiteness of $T[n]$ we can choose $\eta$ small enough so that for each $s, t \in T[n]$ with $s \neq t$, and each $i, j \in\{0,1\}$ with $s^{\frown}\langle i\rangle, t^{\wedge}\langle j\rangle \in T$ :

- $D_{s, 0} \cap D_{s, 1} \cap(\mathbb{R} \times[b, n+1] \times \mathbb{R})=\emptyset$ if $s^{\wedge}\langle 0\rangle, s^{\wedge}\langle 1\rangle \in T$;

- $D_{s, i} \cap D_{t, j}=\emptyset$;

- $E_{s, i} \cap\left(\left[x_{t}, x_{t}^{j}+\eta\right] \times[n, b]\right)=\emptyset$.

The first condition says that if $s$ is a node of $T[n]$ with both immediate extensions in $T$, then $D_{s, 0}$ and $D_{s, 1}$ are disjoint in the semispace $y \geq b$. The second condition says that pinched neighborhoods given by extensions of distinct elements of $T[n]$ are disjoint. The third condition is visualized in figure 10 where, for the sake of the argument, we assume that $x_{s}<x_{t}$, and both $s^{\frown}\langle 0\rangle$ and $s^{\frown}\langle 1\rangle$ are in $T$ : the lighter area is $E_{s, 0} \cup E_{s, 1}$, while the darker area is $\left[x_{t}, x_{t}^{0}+\eta\right] \times[n, b]$.

For every $s \in T[n]$ at least one of $s^{\frown}\langle 0\rangle$ and $s^{\frown}\langle 1\rangle$ belongs to $T$. If only one does, define $\tilde{F}_{T}$ on the relevant $D_{s, i}$ by setting $\left.\tilde{F}_{T}(x, y, z)=\varphi_{s \sim} \sim i\right\rangle^{\prime \prime}(x)$. By VI this definition is compatible with the definition of $\tilde{F}_{T}$ at the previous stage. Notice that $\varphi_{s \smile\langle i\rangle}$ is a solution of $\tilde{F}_{T}$ and no new bifurcation points are added by this definition because $\tilde{F}_{T}$ is Lipschitz (in fact: constant) in the second and third variable.

If both $s^{\frown}\langle 0\rangle$ and $s^{\frown}\langle 1\rangle$ belong to $T$, again the construction is much more delicate. To simplify the notation let $a=x_{s}, a_{0}=x_{s}^{0}, a_{1}=x_{s}^{1}, \psi_{0}=\varphi_{s} \prec\langle 0\rangle\left\lceil\left[x_{s}, a_{0}\right]\right.$ and $\psi_{1}=\varphi_{s^{\sim}}\langle 1\rangle\left\lceil\left[x_{s}, a_{1}\right]\right.$. Furthermore pick $a^{*} \in\left(a, a_{0}\right), b^{*} \in\left(\psi_{1}\left(a^{*}\right), \psi_{0}\left(a^{*}\right)\right)$, and continuous functions $\psi_{2}$ and $\psi_{3}$ with domain respectively $\left[a^{*}, a_{1}\right]$ and $\left[a^{*}, a_{0}\right]$ such that (figure 11 represents the set-up):

1) $\psi_{2}$ and $\psi_{3}$ are $C^{2}$ respectively on $\left(a^{*}, a_{1}\right]$ and $\left(a^{*}, a_{0}\right]$, but

$$
\lim _{x \rightarrow a^{*}} \psi_{2}{ }^{\prime}(x)=\lim _{x \rightarrow a^{*}} \psi_{3}{ }^{\prime}(x)=+\infty ;
$$




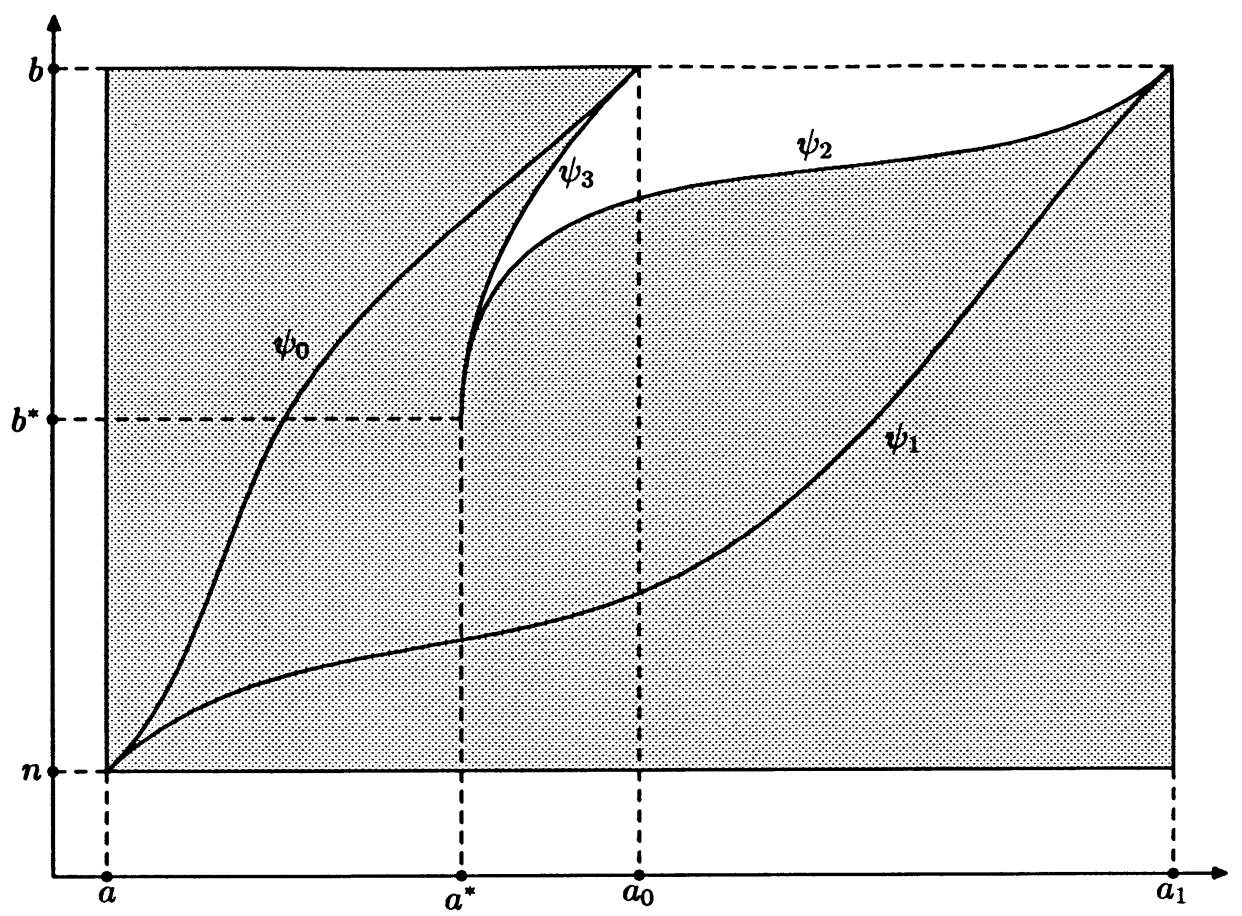

Figure 11.

2) $\psi_{2}$ and $\psi_{3}$ are strictly increasing; $\psi_{2}\left(a^{*}\right)=\psi_{3}\left(a^{*}\right)=b^{*}, \psi_{2}\left(a_{1}\right)=\psi_{3}\left(a_{0}\right)=b$;

3) $\psi_{2}{ }^{\prime}\left(a_{1}\right)=\psi_{1}{ }^{\prime}\left(a_{1}\right)$ and $\psi_{3}{ }^{\prime}\left(a_{0}\right)=\psi_{0}{ }^{\prime}\left(a_{0}\right)$;

4) $\psi_{2}^{\prime \prime}\left(a_{1}\right)=\psi_{1}^{\prime \prime}\left(a_{1}\right)$ and $\psi_{3}^{\prime \prime}\left(a_{0}\right)=\psi_{0}{ }^{\prime \prime}\left(a_{0}\right)$;

$5)$ if $x \in\left(a^{*}, a_{0}\right)$ then $\psi_{1}(x)<\psi_{2}(x)<\psi_{3}(x)<\psi_{0}(x)$;

6) if $x \in\left[a_{0}, a_{1}\right)$ then $\psi_{1}(x)<\psi_{2}(x)$ and $\psi_{1}{ }^{\prime}(x)>\psi_{2}{ }^{\prime}(x)$.

Let

$$
\begin{array}{r}
Q=Q_{s}=\left\{(x, y) \in\left[a, a_{1}\right] \times[n, b] \mid\left(x \in\left[a^{*}, a_{0}\right] \Longrightarrow y \leq \psi_{2}(x) \vee y \geq \psi_{3}(x)\right)\right. \\
\left.\&\left(x \in\left(a_{0}, a_{1}\right] \Longrightarrow y \leq \psi_{2}(x)\right)\right\} \backslash\left\{\left(a^{*}, b^{*}\right)\right\}
\end{array}
$$

( $Q$ is the shaded area in figure 11). Notice that $s \neq t$ implies $Q_{s} \cap Q_{t}=\emptyset$ by our choice of $b$.

Claim 8. There exists a continuous function $G=G_{s}: Q \rightarrow \mathbb{R}$ such that:

7) $G\left(x, \psi_{i}(x)\right)=\psi_{i}{ }^{\prime}(x)$ for $i=0, \ldots, 3$ and $x \in \operatorname{dom}\left(\psi_{i}\right)$.

Moreover if we denote by $S=S_{s}$ the graph of $G$ and let

$$
N=N_{s}=\{(x, y, z)|(x, y) \in Q \&| z-G(x, y) \mid \leq b-y\},
$$

(so that $N$ is a pinched neighborhood of $S$ ), there exists a continuous $\tilde{F}=\tilde{F}_{s}$ : $N \rightarrow \mathbb{R}$ such that the lifting of any solution of $(\tilde{F}, a, n, 1)$ lies on $S, \psi_{0}$ and $\psi_{1}$ are solutions of $(\tilde{F}, a, n, 1)$, and if $\zeta$ is any solution of that Cauchy problem, then either:

i) $\operatorname{dom}(\zeta)=\left[a, a_{0}\right]$ and $\zeta\left(a_{0}\right)=b$, or

ii) $\operatorname{dom}(\zeta)=\left[a, a_{1}\right]$ and $\zeta\left(a_{1}\right)=b$, or 

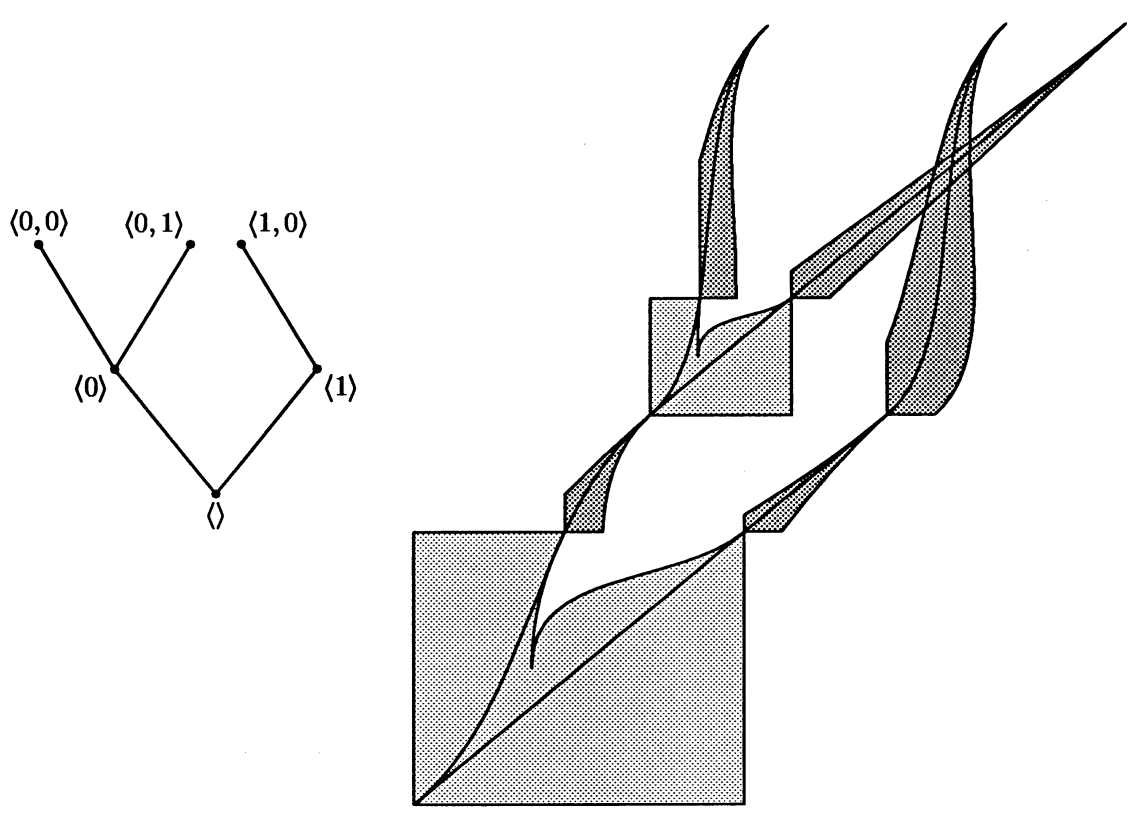

FigURE 12.

iii) $\operatorname{dom}(\zeta)=\left[a, a^{*}\right), \psi_{1}(x)<\zeta(x)<\psi_{0}(x)$ for all $x \in\left(a, a^{*}\right), \lim _{x \rightarrow a^{*}} \zeta(x)=$ $b^{*}$ and $\lim _{x \rightarrow a^{*}} \zeta^{\prime}(x)=+\infty$ (so that $\zeta$ dies without reaching the horizontal line $y=b$ and cannot be further extended to the right).

Notice that the following facts follow from claim $8 \lim _{(x, y) \rightarrow\left(a^{*}, b^{*}\right)} G(x, y)=$ $+\infty, S$ contains the liftings of the $\psi_{i}$ 's and, notwithstanding the fact that $Q$ is not closed in $\mathbb{R}^{2}, S$ is closed in $\mathbb{R}^{3}$. Thus $N$ is also closed in $\mathbb{R}^{3}$.

Obviously this claim is the analogue of claim 6 of section 5 (but there we had $G$ and hence $N$ fixed in advance), but its proof is much more delicate, because we are dealing with a second order differential equation. We postpone the proof of claim 8 to section 8, and complete our construction using the claim.

Claim 8 gives us $N_{s}$ and the definition of $\tilde{F}_{T}$ on $N_{s}$ : again by VI this definition is compatible with the definition of $\tilde{F}_{T}$ at the previous stage. For $i=0,1$ let

$$
\hat{D}_{s, i}=D_{s, i} \cap(\mathbb{R} \times[b, n+1] \times \mathbb{R})
$$

and define $\tilde{F}_{T}$ on $\hat{D}_{s, i}$ by $\tilde{F}_{T}(x, y, z)=\varphi_{s \prec}\langle i\rangle^{\prime \prime}(x)$. There is no conflict because $N_{s} \cap \hat{D}_{s, i}=\left\{\left(a_{i}, b, \psi_{i}{ }^{\prime}\left(a_{i}\right)\right)\right\}$.

Figure 12 represents the projection on the $(x, y)$-plane of the situation up to stage 2 for a tree $T$ with $T[2]=\{\langle 0,0\rangle,\langle 0,1\rangle,\langle 1,0\rangle\}$ (T「2 as a graph is sketched on the left): in the lighter areas we use the $N_{s}$ 's and claim 8 , while in the darker areas we use the $D_{s, i}$ 's or the $\hat{D}_{s, i}$ 's.

Consider the finitely many closed sets (of the form $N_{s}, D_{s, i}$, and $\hat{D}_{s, i}$ ) where $\tilde{F}_{T}$ has just been defined: let $\hat{A}_{n+1}$ be the union of these sets and let $A_{n+1}=B_{n} \cup \hat{A}_{n+1}$. As $\hat{A}_{n+1}$ is closed, so is $A_{n+1}$. Now extend $\tilde{F}_{T}$ to $A_{n+1}$ using Tietze's theorem.

It is easy to check that IVI hold, and this concludes the construction and the proof of theorem 3 . 


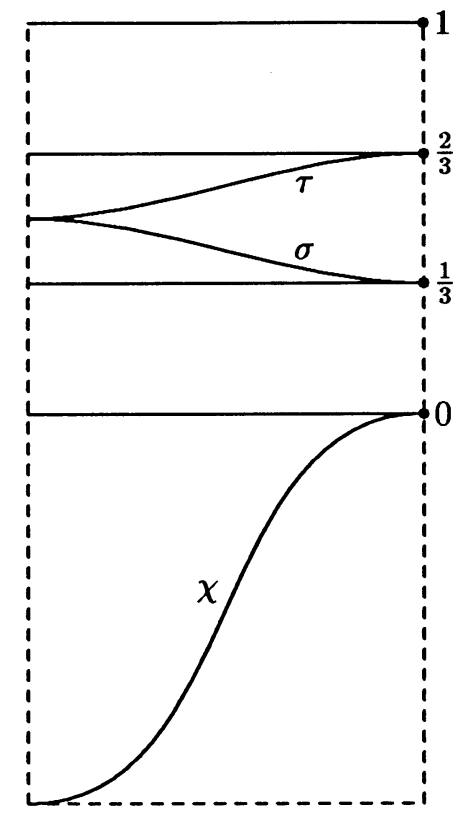

Figure 13.

\section{Proof of the technical Results}

In this section we prove lemma 7 and claim 8

Proof of lemma 7 . The construction we are going to describe is represented in figure 13

Let $\chi:[-1,0] \rightarrow \mathbb{R}$ be $C^{2}$ such that $\chi(-1)=-1, \chi(0)=0$, and $\chi^{\prime}(-1)=$ $\chi^{\prime}(0)=0$. Define $g$ on the lower half of $E$ using lemma 2 applied to $\chi$ and to the constant function 0 , so that C) holds.

For $I=[a, b]$ any segment let $\sigma, \tau:[-1,0] \rightarrow I$ be $C^{2}$ functions such that $\sigma(-1)=\tau(-1)=\frac{1}{2}(a+b), \sigma(0)=a, \tau(0)=b, \sigma(x)<\tau(x)$ for every $x \neq-1$, $\sigma^{\prime}(-1)=\tau^{\prime}(-1)=\sigma^{\prime}(0)=\tau^{\prime}(0)=0$, and $\left|\sigma^{\prime}(x)\right|,\left|\tau^{\prime}(x)\right| \leq b-a$. (In figure $13 a=\frac{1}{3}$ and $b=\frac{2}{3}$.) Let also $a$ and $b$ be the two constant functions. Apply lemma 2 to the pairs $(a, \sigma),(\sigma, \tau)$ and $(\tau, b)$, and use each of the three functions thus obtained in the area between the two relevant curves to obtain a continuous $g_{I}:[-1,0] \times I \rightarrow \mathbb{R}$. The following hold: $\left\|g_{I}\right\| \leq b-a, g_{I}=0$ on $\partial([-1,0] \times I)$ and for every $y \in I$ there is a solution $\varphi$ of $\left(g_{I},-1, y\right)$ such that $\varphi(0)=a$ or $\varphi(0)=b$. Moreover

$\left(\diamond_{I}\right) \quad \forall x \in[-1,0)\left((x, a)\right.$ and $(x, b)$ are not bifurcation points for $\left.g_{I}\right)$.

Define $g$ on $[-1,0] \times[-1,0]$ by

$$
g(x, z)= \begin{cases}g_{I_{s}}(x, z) & \text { if } z \in I_{s} \\ 0 & \text { if } z \in \mathbf{C}\end{cases}
$$

where $I_{s}=\left[\mathbf{c}_{s^{\wedge}\langle 0\rangle}^{1}, \mathbf{c}_{s^{\wedge}\langle 1\rangle}^{0}\right], s \in 2^{<\mathbb{N}}$.

If we check that $g$ is continuous, then $\mathrm{A}$ ) and $(\mathrm{D})$ are immediate and $\mathrm{B}$ ) follows from $\left(\diamond_{I_{s}}\right)$ for every $s \in 2^{<\mathbb{N}}$. 
So let $(x, z) \in E$ : if $z<1$ or if $z$ belongs to the interior of some $I_{s}$, continuity at $(x, z)$ follows by construction. So we may assume $z=\mathbf{c}_{\alpha} \in \mathbf{C}$, so that $g(x, z)=0$. Let $\varepsilon>0$ be given. The set $D=\left\{s \in 2^{<\mathbb{N}} \mid \mathbf{c}_{s^{\frown}\langle\langle 1\rangle}^{0}-\mathbf{c}_{s^{\prime}\langle\langle 0\rangle}^{1} \geq \varepsilon\right\}$ is finite and $\mathbf{c}_{\alpha} \in I_{s}$ for at most one $s \in D$ (in fact $\mathbf{c}_{\alpha} \in I_{s}$ if and only if $\left.\alpha=s^{\frown}\langle 1\rangle\right\urcorner \overline{0}$ or $\alpha=s^{\wedge}\langle 0\rangle^{\wedge} \overline{1}$ ). If such $s \in D$ exists, call it $s_{0}$. Let $\delta>0$ be such that $\delta<d\left(\mathbf{c}_{\alpha}, I_{s}\right)$ for every $s \in D \backslash\left\{s_{0}\right\}$. If $s_{0}$ does exist, by continuity of $g_{I_{s_{0}}}$ we may assume that $\delta$ is so small that $|g(\hat{x}, \hat{z})|<\varepsilon$ for every $(\hat{x}, \hat{z}) \in[-1,0] \times I_{s_{0}}$ such that $d\left((\hat{x}, \hat{z}),\left(x, \mathbf{c}_{\alpha}\right)\right)<\delta$. But then the same holds for every $(\hat{x}, \hat{z}) \in E$ such that $d\left((\hat{x}, \hat{z}),\left(x, \mathbf{c}_{\alpha}\right)\right)<\delta$ : in fact if $\hat{z} \notin I_{s_{0}}$, either $\hat{z} \in \mathbf{C}$, and then $g(\hat{x}, \hat{z})=0$, or else $\hat{z} \in I_{s}$ for some $s \notin D$, and then $|g(\hat{x}, \hat{z})| \leq\left\|g_{I_{s}}\right\| \leq \mathbf{c}_{s^{\frown}\langle 1\rangle}^{0}-\mathbf{c}_{s^{\prec}\langle 0\rangle}^{1}<\varepsilon$.

Before showing claim 8 we prove some technical lemmas.

Lemma 9. Let $A \subseteq \mathbb{R}^{2}$ be open and let $G: A \rightarrow \mathbb{R}$ be $C^{2}$. Let $S \subseteq \mathbb{R}^{3}$ be the graph of $G$. Then there exists a $C^{1}$ function $\tilde{F}: A \times \mathbb{R} \rightarrow \mathbb{R}$ such that:

a) for every $(a, b) \in A$ the lifting of every solution of $(\tilde{F}, a, b, G(a, b))$ lies on $S$;

b) any function whose lifting lies on $S$ is a solution of $\tilde{F}$.

Moreover if $\tilde{F}_{1}: A \times \mathbb{R} \rightarrow \mathbb{R}$ is a continuous function which satisfies a), then $\tilde{F}_{1}$ coincides with $\tilde{F}$ on $S$ and hence $\tilde{F}_{1}$ satisfies also b).

Proof. Let

$$
\tilde{F}(x, y, z)=\frac{\partial G}{\partial x}+z \cdot \frac{\partial G}{\partial y}
$$

so that $\tilde{F}$ is $C^{1}$. If $(a, b) \in A$ let $\zeta=\zeta_{(a, b)}$ be the unique solution of the onedimensional Cauchy problem $(G, a, b) \in \mathbf{C p}$ : the solution is unique because $G$ is $C^{1}$ and the lifting of $\zeta$ lies on $S$ as $\zeta^{\prime}(x)=G(x, \zeta(x))$. Therefore

$$
\zeta^{\prime \prime}(x)=\frac{\partial G}{\partial x}(x, \zeta(x))+\zeta^{\prime}(x) \cdot \frac{\partial G}{\partial y}(x, \zeta(x)) ;
$$

that is: $\zeta$ is a solution of the second order Cauchy problem $(\tilde{F}, a, b, G(a, b)) \in \widetilde{\mathbf{C p}^{2}}$. Moreover $\zeta$ is the only solution, as $\tilde{F}$ is $C^{1}$. This shows that $\tilde{F}$ has the desired property.

On the other hand given $(a, b) \in A$, any $\zeta$ satisfying $\zeta(a)=b$ whose lifting lies on $S$ is a solution of $(G, a, b)$, i.e. $\zeta=\zeta_{(a, b)}$. Therefore if $\tilde{F}_{1}$ is such that the lifting of every solution of $\left(\tilde{F}_{1}, a, b, G(a, b)\right)$ lies on $S$, then

$$
\tilde{F}_{1}(a, b, G(a, b))=\zeta_{(a, b)}{ }^{\prime \prime}(a)=\frac{\partial G}{\partial x}(a, b)+G(a, b) \cdot \frac{\partial G}{\partial y}(a, b),
$$

i.e. $\tilde{F}_{1}(a, b, G(a, b))=\tilde{F}(a, b, G(a, b))$.

The following is the second order analogue of lemma 2

Lemma 10. Suppose $\chi_{0}, \chi_{1}:\left[a, a_{0}\right] \rightarrow \mathbb{R}$ are $C^{2}$ functions such that:

a. $\chi_{0}(a)=\chi_{1}(a), \chi_{0}{ }^{\prime}(a)=\chi_{1}{ }^{\prime}(a)$, and $\chi_{0}{ }^{\prime \prime}(a)=\chi_{1}{ }^{\prime \prime}(a)$;

b. $\chi_{0}{ }^{\prime}(x)<\chi_{1}{ }^{\prime}(x)$ for every $x \in\left(a, a_{0}\right]$ (and hence $\chi_{0}(x)<\chi_{1}(x)$ for every $\left.x \in\left(a, a_{0}\right]\right)$. 
Let

$$
B=\left\{(x, y) \mid x \in\left[a, a_{0}\right] \& y \in\left[\chi_{0}(x), \chi_{1}(x)\right]\right\}
$$

be the region between $\chi_{0}$ and $\chi_{1}$; let also $b=\chi_{0}(a)=\chi_{1}(a)$ and $c=\chi_{0}{ }^{\prime}(a)=\chi_{1}{ }^{\prime}(a)$.

Then there exist a continuous $\tilde{F}:\left[a, a_{0}\right] \times \mathbb{R}^{2} \rightarrow \mathbb{R}$ and a continuous $G: B \rightarrow \mathbb{R}$ such that:

i) $\chi_{0}$ and $\chi_{1}$ are solutions of $(\tilde{F}, a, b, c)$;

ii) $\tilde{F}$ is locally Lipschitz in the second and third variable everywhere but at the points of the form $(a, \bar{b}, c)$; and hence if $(\bar{a}, \bar{c}) \neq(a, c)$, then the Cauchy problem $(\tilde{F}, \bar{a}, \bar{b}, \bar{c})$ has a unique solution;

iii) if $\zeta$ is a solution of $(\tilde{F}, a, b, c)$, then $\chi_{0}(x) \leq \zeta(x) \leq \chi_{1}(x)$ for every $x$;

iv) $G$ is $C^{2}$ on $B \backslash\{(a, b)\}$;

$v)$ the graph of $G$ contains the lifting of any solution of $(\tilde{F}, a, b, c)$.

Proof. Let $F:\left[a, a_{0}\right] \times \mathbb{R} \rightarrow \mathbb{R}$ be the function given by lemma[2] applied to $\chi_{0}{ }^{\prime}$ and $\chi_{1}{ }^{\prime}$, and define $\tilde{F}$ by setting $\tilde{F}(x, y, z)=F(x, z)$. Clearly i) and ii) hold.

If $\zeta$ is a solution of $(\tilde{F}, a, b, c)$, then $\chi_{0}{ }^{\prime}(x) \leq \zeta^{\prime}(x) \leq \chi_{1}{ }^{\prime}(x)$ and hence $\chi_{0}(x) \leq$ $\zeta(x) \leq \chi_{1}(x)$; i.e. iii) holds.

Let $a<x \leq a_{0}$ and $\chi_{0}{ }^{\prime}(x) \leq z \leq \chi_{1}{ }^{\prime}(x)$. Then $(F, x, z)$ has a unique solution $\theta_{(x, z)}$. By the definition of $F$ in the proof of lemma 2

$$
\theta_{(x, z)}(t)=\rho(x, z) \cdot \chi_{0}{ }^{\prime}(t)+(1-\rho(x, z)) \cdot \chi_{1}{ }^{\prime}(t)
$$

where $\rho(x, z)=\frac{z-\chi_{1}{ }^{\prime}(x)}{\chi_{0}{ }^{\prime}(x)-\chi_{1}{ }^{\prime}(x)}$. Let $B^{\prime}=\left\{(x, z) \mid \chi_{0}{ }^{\prime}(x) \leq z \leq \chi_{1}{ }^{\prime}(x)\right\}$, and define $H: B^{\prime} \rightarrow \mathbb{R}$ by letting $H(a, c)=b$ and, for $x \neq a$,

$$
\begin{aligned}
H(x, z) & =b+\int_{a}^{x} \theta_{(x, z)}(t) d t \\
& =b+\rho(x, z) \cdot \chi_{0}(x)+(1-\rho(x, z)) \cdot \chi_{1}(x) .
\end{aligned}
$$

It is easy to check that $H$ is continuous on $B^{\prime}$ and $C^{2}$ on $B^{\prime} \backslash\{(a, c)\}$. Moreover

$$
\frac{\partial H}{\partial z}(x, z)=\frac{\chi_{1}(x)-\chi_{0}(x)}{\chi_{1}{ }^{\prime}(x)-\chi_{0}{ }^{\prime}(x)} \neq 0
$$

for all $x \neq a$. Hence there exists $G: B \rightarrow \mathbb{R}$ such that $G(x, H(x, z))=z$ and $G$ satisfies iv).

To check that $G$ satisfies also v), suppose $\zeta$ is a solution of $(\tilde{F}, a, b, c)$, so that $\zeta^{\prime}$ is a solution of $(F, a, c)$ and for every $x \in\left(a, a_{0}\right]$ we have $\zeta^{\prime}=\theta_{\left(x, \zeta^{\prime}(x)\right)}$. Therefore

$$
\begin{aligned}
G(x, \zeta(x)) & =G\left(x, b+\int_{a}^{x} \theta_{\left(x, \zeta^{\prime}(x)\right)}(t) d t\right) \\
& =G\left(x, H\left(x, \zeta^{\prime}(x)\right)\right) \\
& =\zeta^{\prime}(x)
\end{aligned}
$$

holds for every $x \in\left(a, a_{0}\right]$ and $\left.\mathrm{v}\right)$ is proved.

Proof of claim 8. To prove claim 8 we will use lemmas 10] and 9. Pick $a_{2} \in\left(a, a^{*}\right)$ such that $\psi_{1}^{\prime}(x)<\psi_{0}^{\prime}(x)$ for all $x \in\left(a, a_{2}\right]$; similarly pick $a_{3} \in\left(a^{*}, a_{0}\right)$ such that $\psi_{0}{ }^{\prime}(x)<\psi_{3}{ }^{\prime}(x)$ for all $x \in\left(a_{3}, a_{0}\right]$ and $\psi_{2}{ }^{\prime}(x)<\psi_{1}{ }^{\prime}(x)$ for all $x \in\left(a_{3}, a_{1}\right]$ ( $a_{3}$ exists by condition 6$)$ before the statement of the claim). Figures 11 and 14 represent the situation. 


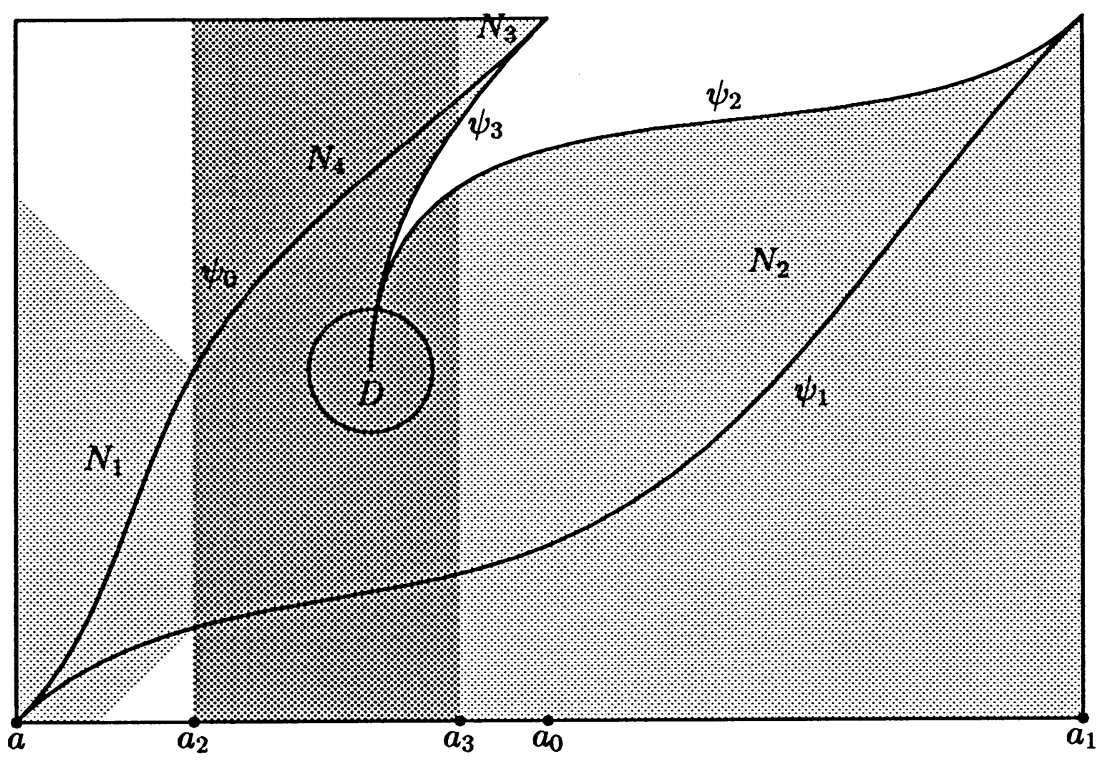

FigURE 14.

Lemma 10 is applied three timet 2 to the pairs of functions $\left(\psi_{1}, \psi_{0}\right)$ on $\left[a, a_{2}\right]$, $\left(\psi_{1}, \psi_{2}\right)$ on $\left[a_{3}, a_{1}\right]$, and $\left(\psi_{3}, \psi_{0}\right)$ on $\left[a_{3}, a_{0}\right]$ to obtain continuous $\tilde{F}_{1}, \tilde{F}_{2}$, and $\tilde{F}_{3}$ and $G_{1}, G_{2}$, and $G_{3}$ with domain $B_{1}, B_{2}$, and $B_{3}$ respectively. Thus $B_{1}$ is the region between $\psi_{0}$ and $\psi_{1}$ with $x \in\left[a, a_{2}\right], B_{2}$ is the region between $\psi_{1}$ and $\psi_{2}$ with $x \in\left[a_{3}, a_{1}\right]$, and $B_{3}$ is the region between $\psi_{0}$ and $\psi_{3}$ with $x \in\left[a_{3}, a_{0}\right]$. Since $B_{1}, B_{2}$ and $B_{3}$ are pairwise disjoint we can consider the union of $G_{1}, G_{2}$ and $G_{3}$ as a single function $G_{0} . \tilde{F}_{1}$ is defined on $\left[a, a_{2}\right] \times \mathbb{R}^{2}$, but we will restrict it to the pinched neighborhood of $S_{1}$

$$
N_{1}=\left\{(x, y, z) \mid(x, y) \in\left[a, a_{2}\right] \times[n, b] \& d\left((x, y, z), S_{1}\right) \leq a_{2}-x\right\},
$$

where $S_{1}=\operatorname{graph}\left(G_{1}\right)$. We also restrict $\tilde{F}_{2}$ and $\tilde{F}_{3}$ to

$$
\begin{aligned}
& N_{2}=\left\{(x, y, z) \mid(x, y) \in\left[a_{3}, a_{1}\right] \times[n, b] \& y \leq \psi_{2}(x)\right\}, \\
& N_{3}=\left\{(x, y, z) \mid(x, y) \in\left[a_{3}, a_{0}\right] \times[n, b] \& y \geq \psi_{3}(x)\right\}
\end{aligned}
$$

respectively. In figure 14 the projections on the $(x, y)$-plane of $N_{1}, N_{2}$ and $N_{3}$ are the lightly shaded areas.

Let $M=\mathbb{R}^{2} \backslash\left\{(a, n),\left(a^{*}, b^{*}\right),\left(a_{0}, b\right),\left(a_{1}, b\right)\right\}$ and $C=Q \cap M \cap\left(B_{1} \cup B_{2} \cup B_{3}\right)$. $C$ is a closed regular submanifold of the manifold $M$ and $G_{0}$ is $C^{2}$ on $C$. Let $D$ be a closed neighborhood of $\left(a^{*}, b^{*}\right)$ disjoint from $C$. Define $G_{0}^{*}$ on $D \backslash\left\{\left(a^{*}, b^{*}\right)\right\}$ by $G_{0}^{*}(x, y)=\left[\left(x-a^{*}\right)^{2}+\left(y-b^{*}\right)^{2}\right]^{-1}$, so that $\lim _{(x, y) \rightarrow\left(a^{*}, b^{*}\right)} G_{0}^{*}(x, y)=+\infty$, $G_{0}^{*}$ is $C^{2}$ and its domain is a closed regular submanifold of $M$. We now extend $G_{0} \cup G_{0}^{*}$ to a $C^{2}$ function $G$ defined on $M$, using the analogue of Tietze's theorem

\footnotetext{
${ }^{2}$ For the last two pairs the situation is reversed with respect to lemma 10 because the point where the two functions coincide is the upper (and not the lower) bound of the interval. We have also (as is obviously needed) the reverse inequality between the derivatives. This is a straightforward variant of the lemma as stated.
} 
for differentiable maps (see e.g. V.4.1 of [2], where the following is stated for $C^{\infty}$ functions):

Lemma 11. Let $M$ be a $C^{2}$ manifold and $A \subseteq M$ be a closed regular submanifold. If $G: A \rightarrow \mathbb{R}$ is $C^{2}$, then there exists $H: M \rightarrow \mathbb{R}$ which is $C^{2}$ and extends $G$.

By lemma 9 there exists a $C^{1}$ function $\tilde{F}_{4}$ with domain $M \times \mathbb{R}$ such that the lifting of any solution of $\tilde{F}_{4}$ lies on the graph of $G$. Since the same holds (within their respective domains) for $\tilde{F}_{1}, \tilde{F}_{2}$ and $\tilde{F}_{3}$, lemma 9 implies that they coincide with $\tilde{F}_{4}$ on the graph of $G_{0}$. Let

$$
N_{4}=\left\{(x, y, z)\left|x \in\left[a_{2}, a_{3}\right] \&(x, y) \in Q \&\right| z-G(x, y) \mid \leq a_{3}-x\right\}
$$

be a pinched neighborhood of a portion of the graph of $G$. $N_{4}$ is closed in $\mathbb{R}^{3}$. In figure 14 the projection on the $(x, y)$-plane of $N_{4}$ is the darkest area.

Let $\tilde{F}: N_{1} \cup \cdots \cup N_{4} \rightarrow \mathbb{R}$ be defined by $\tilde{F}(x, y, z)=\tilde{F}_{i}(x, y, z)$ if $(x, y, z) \in N_{i}$. $\tilde{F}$ is well defined as $\tilde{F}_{i}$ agrees with $\tilde{F}_{j}$ on $N_{i} \cap N_{j}$ : e.g. $N_{1} \cap N_{4}=\left\{\left(a_{2}, y, G\left(a_{2}, y\right)\right) \mid\right.$ $\left.y \in\left[\psi_{1}\left(a_{2}\right), \psi_{0}\left(a_{2}\right)\right]\right\}$ is a subset of $\operatorname{graph}\left(G_{0}\right)$, where $\tilde{F}_{1}$ and $\tilde{F}_{4}$ coincide. As $N_{1} \cup \cdots \cup N_{4}$ is closed, $\tilde{F}$ can be extended to $\mathbb{R}^{3}$.

$G \vdash Q$ is the other function whose existence was asserted by claim 8 , We are left with proving that our construction actually works. Let $S=\operatorname{graph}(G\lceil Q)$. It is clear that $\psi_{0}$ and $\psi_{1}$ are solutions of the Cauchy problem $(\tilde{F}, a, n, 1)$. Let $\zeta$ be a solution of $(\tilde{F}, a, n, 1)$. By the properties of $\tilde{F}_{1}$ we have $\zeta\left(a_{2}\right) \in\left[\psi_{1}\left(a_{2}\right), \psi_{0}\left(a_{2}\right)\right]$ and the lifting of $\zeta$ lies on $S$. In the interval $\left[a_{2}, a_{3}\right], \zeta$ is a solution of $\tilde{F}_{4}$ and hence its lifting does not leave $S$. There are three possibilities. If $\lim _{x \rightarrow a^{*}} \zeta(x)=b^{*}$, then $\lim _{x \rightarrow a^{*}} \zeta^{\prime}(x)=+\infty$ and we are in case iii). If $\lim _{x \rightarrow a^{*}} \zeta(x)>b^{*}$, then $\zeta$ does not die and $\zeta\left(a_{3}\right) \in\left[\psi_{3}\left(a_{3}\right), \psi_{0}\left(a_{3}\right)\right]$. In this case, by the properties of $\tilde{F}_{3}$ we have $\zeta\left(a_{0}\right)=b$ and we are in case i). If $\lim _{x \rightarrow a^{*}} \zeta(x)<b^{*}$, a similar argument leads to case ii).

\section{REFERENCES}

[1] A. Andretta and A. Marcone, Ordinary differential equations and descriptive set theory: uniqueness and globality of solutions of Cauchy problems in one dimension, Fundamenta Mathematicae 153 (1997), 157-190. MR 98g:34009

[2] W. M. Boothby, An Introduction to Differentiable Manifolds and Riemannian Geometry, second edition, Academic Press, 1986. MR 87k:58001

[3] A. S. Kechris, Classical Descriptive Set Theory, Springer-Verlag, 1995. MR 96e:03057

Dipartimento di Matematica, Università di Torino, via Carlo Alberto 10, 10123 TORINO, ITALY

E-mail address: andretta@dm.unito.it

Dipartimento di Matematica, Università di Torino, via Carlo Alberto 10, 10123 TORINO, ITALY

Current address: Dipartimento di Matematica e Informatica, Università di Udine, viale delle Scienze 206, 33100 Udine, Italy

E-mail address: marcone@dimi.uniud.it 